\title{
A multi-wavelength study of the young star V1118 Orionis in outburst ${ }^{\star}, \star \star$
}

\author{
M. Audard ${ }^{1,2}$, G. S. Stringfellow ${ }^{3}$, M. Güdel ${ }^{4}$, S. L. Skinner ${ }^{3}$, F. M. Walter ${ }^{5}$, E. F. Guinan ${ }^{6}$, R. T. Hamilton ${ }^{6,7}$ \\ K. R. Briggs ${ }^{4}$, and C. Baldovin-Saavedra ${ }^{1,2}$ \\ 1 ISDC Data Center for Astrophysics, University of Geneva, Ch. d'Ecogia 16, 1290 Versoix, Switzerland \\ e-mail: Marc. Audard@unige.ch \\ 2 Observatoire de Genève, University of Geneva, Ch. des Maillettes 51, 1290 Versoix, Switzerland \\ 3 Center for Astrophysics and Space Astronomy, University of Colorado, Boulder, CO 80309-0389, USA \\ ${ }^{4}$ Institut für Astronomie, ETH Zürich, 8093 Zürich, Switzerland \\ 5 Department of Physics and Astronomy, Stony Brook University, Stony Brook, NY 11794-3800, USA \\ 6 Department of Astronomy and Astrophysics, Villanova University, Villanova 19085, PA, USA \\ 7 Department of Astronomy, New Mexico State University, 320 East Union Ave, Apt. 1434, Las Cruces, NM 88001, USA
}

Received 31 July 2009 / Accepted 16 December 2009

\section{ABSTRACT}

Context. The accretion history of low-mass young stars is not smooth but shows spikes of accretion that can last from months and years to decades and centuries.

Aims. Observations of young stars in outbursts can help us understand the temporal evolution of accreting stars and the interplay between the accretion disk and the stellar magnetosphere.

Methods. The young late-type star V1118 Orionis was in outburst from 2005 to 2006. We followed the outburst with optical and near-infrared photometry. The X-ray emission was further probed with observations taken with XMM-Newton and Chandra during and after the outburst. In addition, we obtained mid-infrared photometry and spectroscopy with Spitzer at the peak of the outburst and in the post-outburst phase.

Results. The spectral energy distribution of V1118 Ori varied significantly over the course of the outburst. The optical flux showed the largest variations, most likely caused by enhanced emission by a hot spot. The hot spot dominated the optical and near-infrared emission at the peak of the outburst, while the disk emission dominated in the mid-infrared. The emission silicate feature in V1118 Ori is flat and does not vary in shape, but was slightly brighter at the peak of the outburst compared to the post-outburst spectrum. The $\mathrm{X}$-ray flux correlated with the optical and infrared fluxes, indicating that accretion affected the magnetically active corona and the stellar magnetosphere. The thermal structure of the corona was variable with some indication of a cooling of the coronal temperature in the early phase of the outburst with a gradual return to normal values. Color-color diagrams in the optical and infrared showed variations during the outburst, with no obvious signature of reddening caused by circumstellar matter. Using Monte-Carlo realizations of star+disk+hotspot models to fit the spectral energy distributions in "quiescence" and at the peak of the outburst, we determined that the mass accretion rate varied from about $2.5 \times 10^{-7} M_{\odot} \mathrm{yr}^{-1}$ to $1.0 \times 10^{-6} M_{\odot} \mathrm{yr}^{-1}$; in addition, the fractional area of the hotspot increased significantly.

Conclusions. The multi-wavelength study of the V1118 Ori outburst helped us to understand the variations in spectral energy distributions and demonstrated the interplay between the disk and the stellar magnetosphere in a young, strongly accreting star.

Key words. accretion, accretion disks - infrared: stars - circumstellar matter - stars: coronae - stars: pre-main-sequence X-rays: stars

\section{Introduction}

The star formation process involves strong accretion of circumstellar matter onto the protostar. The time evolution of the mass accretion rate is of deep interest for understanding the timescale of stellar growth and the lifetime of proto-planetary disks. While the mass accretion rate in young stars overall decreases with increasing stellar age (Hartmann et al. 1998), it can also show significant and rapid changes over time (e.g., Hartmann et al. 1993). A handful of accreting young stars

* Appendices are only available in electronic form at http://www. aanda.org

$\star \star$ Two tables of photometry are only available in electronic form at the CDS via anonymous ftp to

cdsarc.u-strasbg.fr $(130.79 .128 .5)$ or via

http://cdsweb.u-strasbg.fr/cgi-bin/qcat? J/A+A/511/A63 display powerful eruptive events with flux increases in the optical regime of a few magnitudes. Two classes have emerged: FUors, which display outbursts of 4 mag and more, last several decades, and hence, show a low recurrence rate. EXors (named after the prototype EX Lup), in contrast, show somewhat smaller outbursts $(\Delta V=2-3 \mathrm{mag})$ on a much shorter timescale, from a few months to a few years, and may occur repeatedly (see review by Hartmann \& Kenyon 1996; also Herbig 2008). Such outbursts are believed to originate during a rapid increase in the disk accretion rate over a short period of time, from values of $10^{-7} M_{\odot} \mathrm{yr}^{-1}$ to $10^{-4} M_{\odot} \mathrm{yr}^{-1}$, although the underlying cause of such an increase in mass accretion rate is unclear (thermal disk instabilities, Lin \& Papaloizou 1985; Bell \& Lin 1994; close companions, Bonnell \& Bastien 1992; cluster-induced encounters, Pfalzner et al. 2008; giant planets in the disk, Clarke \& Syer 1996; Lodato \& Clarke 2004; a combination of gravitational 
instability and the triggering of the magnetorotational instability, Armitage et al. 2001; accretion of clumps in a gravitationally unstable disk, Vorobyov \& Basu 2005, 2006). The limited number of eruptive young stars and the long recurrence time (especially for FUor-type objects) make it difficult to test models. It is, therefore, important to study the evolution of outbursts in as much detail as possible and to understand the place of outbursting young stars in the evolutionary scheme from highly accreting, embedded protostars to "normal" accreting classical T Tauri stars (CTTS).

The optically revealed CTTS can make excellent comparison stars to outbursting young stars. They have been studied extensively in the past: optical and infrared observations have given clues about the presence of accretion disks, on the mass accretion rates, and disk winds, while millimeter observations provided constraints on the dust disk mass and on $\mathrm{CO}$ outflows. In the X-rays, the origin of emission in young, accreting stars has gained significant attention in the last few years. Kastner et al. (2002) obtained the first X-ray grating spectrum of TW Hya, a moderately accreting CTTS, and observed unexpected new spectral features: the O VII and Ne IX He-like triplets showed very low forbidden-to-intercombination line ratios and indicated high electron densities on the order of $10^{12}-10^{13} \mathrm{~cm}^{-3}$. The effect of UV photoexcitation on the observed line ratio was deemed negligible. In addition, the X-ray spectrum was consistent with a quasi-isothermal plasma of about 3 MK. Kastner et al. (2002) concluded that the X-ray emission in TW Hya was due to accretion, a result further supported by Stelzer \& Schmitt (2004) and Ness \& Schmitt (2005). Additional high-resolution X-ray spectra of CTTS have also indicated that accretion may play a significant role for X-rays in accreting stars (Schmitt et al. 2005; Günther et al. 2006; Robrade \& Schmitt 2006; Argiroffi et al. 2007; Günther et al. 2007; Sacco et al. 2008). Soft X-ray emission from shocks in jets may also be detected (Güdel et al. 2005; Güdel et al. 2008; Kastner et al. 2005; Robrade \& Schmitt 2007; Schneider \& Schmitt 2008; Günther \& Schmitt 2009). Telleschi et al. (2007) and Güdel \& Telleschi (2007) showed that CTTS display a soft X-ray excess from plasma at low temperature. Such a plasma (mostly detected in the O VII lines) is difficult to detect with CCD spectroscopy but can easily be revealed by high-resolution X-ray spectra when the absorbing column density is not too high. The soft X-ray excess can co-exist with the hot plasma observed in the vast majority of young stars, which is due to scaled-up solar-like magnetic activity (Smith et al. 2005; Audard et al. 2005a; Preibisch et al. 2005; Telleschi et al. 2007). The origin of such an excess is unclear, but it could be due in part to accretion onto the stellar photosphere. It is crucial to understand how the X-ray emission in young stars, in particular those actively accreting matter, can be influenced by the accretion process. FUor and EXor stars are, therefore, the ideal cases for understanding the physical mechanism common to both CTTS and outbursting stars.

In recent years, several young low-mass stars have been observed in outburst, in the optical and infrared, but also in X-rays: V1647 Ori in 2003-2005 (e.g., Kastner et al. 2004, Grosso et al. 2005, Kastner et al. 2006a; see also Aspin et al. 2008, and references therein) and recently in 2008-2009 (Itagaki 2008; Aspin 2008; Venkat \& Anandarao 2008; Aspin et al. 2009), V1118 Ori in 2005-2006 (Audard et al. 2005b; Lorenzetti et al. 2006, 2007; Herbig 2008). Kastner et al. (2006b) also observed a young stellar object in eruption in LDN 1415 with Chandra but failed to detect it. EX Lup also showed an extreme outburst in early 2008 (Jones 2008a,b; Kospal et al. 2008). Ábrahám et al. (2009) detected crystalline features in the silicate feature of EX Lup in outburst which were not present in the pre-outburst spectrum, suggesting crystallization by thermal annealing in the surface layer of the inner disk. X-ray observations were obtained with Chandra (PI: Weintraub) and Swift (PI: Stringfellow) but have not yet been published. The FUor objects FU Ori, V1735 Cyg, and $\mathrm{Z}$ CMa have also been detected in X-rays (Skinner et al. 2006, 2009a; Stelzer et al. 2009).

The V1647 Ori campaign showed an increase in X-ray flux by a factor of 200 from the pre-outburst flux, in line with the flux increase in the infrared. The X-ray flux then followed the optical outburst flux and returned to its pre-outburst level after the outburst ended (Kastner et al. 2006a). The initial observations of V1118 Ori in X-rays revealed different behavior, with little flux enhancement (Audard et al. 2005b), but also indicated that the rapid increase of accretion rate in outbursts can impact the $\mathrm{X}$-ray emission of young accreting stars. The present paper aims to present the remainder of the X-ray data of V1118 Ori taken during and after the 2005-2006 outburst, together with contemporaneous optical and infrared data.

\section{The young erupting star V1118 Ori}

The outburst of V1118 Ori, a young low-mass M1e star in the Orion Nebula ( $d=400$ pc; Muench et al. 2008 for a discussion), was reported in early January 2005 by Williams et al. (2005). Hillenbrand (1997) and Stassun et al. (1999) provide details about its physical properties: $M_{\star}=0.41 M_{\odot} ; R_{\star}=1.29 R_{\odot}$; $P_{\text {rot }}=2.23 \pm 0.04 \mathrm{~d} ; L_{\text {bol }} \geq 0.25 L_{\odot} ; \log T_{\text {eff }}[\mathrm{K}]=3.562$; $\log t[\mathrm{yr}]=6.28$. Recently, Reipurth et al. (2007) resolved V1118 Ori into a close binary separated by $0 . ' 18$ with a position angle of $329^{\circ}$ and a magnitude difference of $\Delta m=0.4$ mag in the $\mathrm{H} \alpha$-band image. The observation was obtained on 2004 Jan. 29, thus before the 2005-2006 outburst (see Fig. 6). The binarity of V1118 Ori leaves unclear which star actually erupted. However, the small magnitude difference indicates that the components are similar, suggesting similar effective temperatures. We may also assume that they show similar disk evolution. While the binarity of V1118 Ori complicates the interpretation of the combined photometry and spectroscopy in quiescence, this should have little impact on the interpretation of the outburst data, as only one star+disk component dominates the emission. In this paper, we have used the above stellar properties that assumed a single star.

V1118 Ori has shown frequent outbursts (e.g., 1983-84, 1988-90, 1992-94, 1997-98; see Garcia \& Parsamian 2000; and Herbig 2008 for details). Indeed, after returning to quiescence in mid-2006, V1118 Ori had another outburst in late 2007 (Garcia \& Parsamian 2008). We focus here on our monitoring campaign to study the 2005-2006 outburst of V1118 Ori in the $\mathrm{X}$-rays, optical and infrared. Additional properties in the optical and infrared were obtained independently during and after the outburst by Lorenzetti et al. (2006, 2007) and Herbig (2008). Using wind models, Lorenzetti et al. (2006) derived a mass loss rate of $4 \times 10^{-8} M_{\odot} \mathrm{yr}^{-1}$ from the $\mathrm{H}$ I recombination line, and (3-8) $\times 10^{-7} M_{\odot} \mathrm{yr}^{-1}$ from the $\mathrm{CO}$ emission at $2.3 \mu \mathrm{m}$ for the neutral molecular gas; they also found no evidence of infrared cooling from a collimated jet or outflow. Lorenzetti et al. (2007) also found evidence of intrinsic polarization in the $I$ band, and irregular fluctuations during the outburst. Herbig (2008) obtained Keck/HIRES spectra of V1118 Ori in the decaying phase of the outburst and in the post-outburst phase. He noted the detection of Li I 16707 in emission during the outburst, in contrast to the absorbed feature in CTTS spectra. The feature, however, was seen in absorption after the outburst. Similar behavior was exhibited by the K I lines at $\lambda \lambda 7664$ and $7698 \AA$. A P Cyg profile was also 
Table 1. X-ray observation log.

\begin{tabular}{|c|c|c|c|c|c|c|}
\hline Parameter & Sep. 2002 & & Jan. 2005 & Feb. 2005 & Mar. 2005 & Sep. 2005 \\
\hline Satellite & Chandra & & Chandra & XMM-Newton & XMM-Newton & XMM-Newton \\
\hline ObsID & 2548 & & 6204 & 0212480301 & 0212480401 & 0212481101 \\
\hline Duration $(\mathrm{ks})$ & 48 & & 5 & 20 & 20 & 20 \\
\hline Observation date & 2002 Sep. 6-7 & & 2005 Jan. 26 & 2005 Feb. $18-19$ & 2005 Mar. 21 & 2005 Sep. 8 \\
\hline UT & $12: 57-02: 54$ & & 03:07-05:04 & $22: 36-04: 50$ & $16: 20-22: 08$ & $12: 43-18: 51$ \\
\hline \multirow[t]{2}{*}{ Average JD-2 450000} & 2524.3 & & 3396.7 & 3420.6 & 3451.3 & 3622.2 \\
\hline & Jan. 2006 & Feb. 2006 & Mar. 2006 & Apr. 2006 & Jul. 2006 & Dec. 2007 \\
\hline Satellite & Chandra & Chandra & XMM-Newton & Chandra & Chandra & Chandra \\
\hline ObsID & 6416 & 6417 & 0403200101 & 6418 & 6419 & 8936 \\
\hline Duration (ks) & 30 & 30 & 100 & 30 & 30 & 37.5 \\
\hline Observation date & 2006 Jan. 4-5 & 2006 Feb. 23 & 2006 Mar. 2-3 & 2006 Apr. 23 & 2006 Jul. 24 & 2007 Dec. $14-15$ \\
\hline UT & $18: 33-03: 09$ & 09:41-17:47 & 19:02-21:06 & $14: 42-23: 31$ & $07: 50-16: 49$ & $14: 49-01: 13$ \\
\hline Average JD-2 450000 & 3740.5 & 3790.2 & 3797.8 & 3849.3 & 3941.0 & 4449.3 \\
\hline
\end{tabular}

found in $\mathrm{H} \alpha$ during the outburst, which disappeared thereafter. In the near-infrared, Lorenzetti et al. (2007) also found that the emission lines detected during the outburst (H I, He I, CO $2.3 \mu \mathrm{m}$ band, a few neutral metals) disappeared about a year later.

In the X-ray regime, the initial Jan.-Mar. 2005 data were published by Audard et al. (2005b). In brief, the X-ray data of early 2005 indicated that the X-ray flux and luminosity varied by less than a factor of two during the outburst, at the same level as in a pre-outburst observation in 2002. The fluxes in the optical and near-infrared varied more significantly, by factors of 2-10. The hydrogen column density showed no evidence for variation from its modest pre-outburst value of $N_{\mathrm{H}} \approx 3 \times 10^{21} \mathrm{~cm}^{-2}$. However, there was evidence of a spectral change from a dominant hot plasma $(\approx 25 \mathrm{MK})$ in 2002 and in January 2005 to a cooler plasma $(\approx 8 \mathrm{MK})$ in February 2005 and probably in March 2005. We hypothesized that the hot magnetic loops high in the corona were disrupted by the closing in of the accretion disk as a consequence of the increased accretion rate during the outburst, whereas the lower cooler loops were probably less affected and became the dominant coronal component (Audard et al. 2005b). We argued that the cool component in V1118 Ori could not originate from shocks because free-fall velocities of matter falling from the truncation radius are too low (see Audard et al. 2005b for further discussion). In a subsequent paper, Lorenzetti et al. (2006) independently analyzed our public data sets of early 2005, and also included the September 2005 XMM-Newton observation, together with their near-infrared data sets. The September 2005 observation showed a decrease in $\mathrm{X}$-ray flux at the start of the decay phase.

The initial paper by Audard et al. (2005b) presented and analyzed the outburst data through March 2005. We present here the XMM-Newton and Chandra data obtained from September 2005 on, which are analyzed in the context of our multiwavelength analysis. We also include a post-outburst data set obtained by Chandra in December 2007. The latter was taken during a minor outburst detected in the optical (Garcia \& Parsamian 2008). Extensive optical and near-infrared photometry are presented and analyzed, obtained from our team's imaging data, along with mid-infrared photometry and spectroscopy with Spitzer.

\section{Observations and data reduction}

\subsection{X-ray}

Table 1 provides the observation log of our 2005-2006 monitoring campaign of the outburst of V1118 Ori with XMM-Newton
(Jansen et al. 2001) and Chandra (Weisskopf et al. 1996). We also provide information about the 2002 serendipitous observation of V1118 Ori (an observation in 2001 with XMM-Newton was reported in Audard et al. 2005b but is not mentioned here since the star was not detected). A deep XMM-Newton observation was obtained in early 2006 that complements the short monitoring observations. Finally, we include the post-outburst Chandra observation in December 2007 as well. The angular resolution of neither X-ray satellites was high enough to separate the V1118 Ori binary.

The XMM-Newton data were processed with SAS 7.0. Standard procedures were applied. We used an extraction circle of radius $20^{\prime \prime}$ for the source and a nearby circular region of $60^{\prime \prime}$ radius (40" for Sep. 2005 and 35" for Mar. 2006) for the background. Only event patterns lower than 4 and 12 were used for the European Photon Imaging Cameras (EPIC) pn and MOS (Strüder et al. 2001; Turner et al. 2001), respectively. The background flux levels were high during all XMM-Newton observations, in particular in March and September 2005. As described in Audard et al. (2005b), we used only the MOS1 and MOS2 data for the March 2005 observation. The September 2005 observation was so affected by the background that we lost about half the exposure time in the EPIC pn, while no MOS data were available. The deep March 2006 observation was affected by a system failure at the Mission Operations Centre and the EPIC pn experienced full scientific buffer in the last part of the observation, explaining the reduced exposure time in the EPIC pn (78 ks) compared to the EPIC MOS (90 ks). No Optical Monitor data were taken with the XMM-Newton X-ray observation because of the presence of the nearby bright Trapezium stars.

The Chandra data were processed with CIAO 3.3 and CALDB 3.2.3 ${ }^{1}$. The task psextract was used to extract the spectra for V1118 Ori and the nearby background. We used a circle of $2^{\prime \prime}$ radius ( $\approx 4$ pixels) for the star and an annulus centered at the position of the star but with radii of 10 and 60 pixels for the background; our background area was, therefore, 212 times larger than the source extraction area. Our extraction radius for the star includes $95 \%$ of the encircled energy at $1.5 \mathrm{keV}$ and $90 \%$ at $4.5 \mathrm{keV}$. For the January 2005 observation in sub-array mode, we used an outer radius of 40 pixels for the background annulus. For the 2002 observation, we used circles of radii of $15^{\prime \prime}$ and $45^{\prime \prime}$ for the source and the background, respectively (see Audard et al. 2005b).

\footnotetext{
1 We used the pipeline data for the December 2007 observation, which was calibrated with CALDB 3.4.2.
} 
Table 2. Spitzer IRAC and MIPS flux density measurements (units of mJy).

\begin{tabular}{crrrrr}
\hline \hline Date & $3.6 \mu \mathrm{m}$ & $4.5 \mu \mathrm{m}$ & $5.8 \mu \mathrm{m}$ & $8.0 \mu \mathrm{m}$ & $24 \mu \mathrm{m}$ \\
\hline 2004 Mar. 09 & $28.6 \pm 0.1$ & $36.3 \pm 0.1$ & $39.2 \pm 1.3$ & $54.8 \pm 0.5$ & \\
2004 Mar. 20 & & & & & $74.2 \pm 0.5$ \\
2004 Oct. 12 & $46.5 \pm 0.2$ & $50.6 \pm 0.2$ & $53.3 \pm 0.3$ & $55.9 \pm 0.5$ & \\
2004 Oct. 27 & & $43.4 \pm 0.1$ & & $57.5 \pm 0.3$ & \\
2005 Feb. 20 & $143.3 \pm 1.7$ & $169.7 \pm 2.0$ & $148.2 \pm 4.9$ & $144.1 \pm 1.9$ & \\
2005 Mar. 28 & $173.7 \pm 1.8$ & $203.9 \pm 2.0$ & $194.1 \pm 5.0$ & $167.6 \pm 1.9$ & \\
2007 Oct. 21 & $42.4 \pm 1.7$ & $48.7 \pm 1.9$ & $52.2 \pm 4.9$ & $53.1 \pm 1.8$ & \\
\hline
\end{tabular}

\subsection{Optical and near-infrared}

\subsubsection{SMARTS}

We observed V1118 Ori with the ANDICAM dual-channel imager on the SMARTS/CTIO ${ }^{2} 1.3 \mathrm{~m}$ telescope on 115 nights. Such dense coverage was made possible by the service mode operations employed by the SMARTS consortium. We have 5 pre-outburst observations (2004 Feb. 2 through 2004 Apr. 8) that can be used to set the quiescent flux levels. The intensive monitoring began 2005 Jan. 10, shortly after the outburst was reported. There were no observations from 2005 Apr. 4 through 2005 Jul. 31 due to proximity to the Sun. We observed with cadences between 1 per day and 1 per week, with observations generally every 3-4 days. Our intensive monitoring terminated on 2006 May 6. We also followed the decay of the second outburst from 2007 Dec. 27 through 2008 Mar. 27. Details of the data reduction are given in Appendix A. Table A.2 (available online only) gives the nightly average photometry for SMARTS.

\subsubsection{Villanova}

Photometric coverage in the optical (standard Bessel VRI) was obtained with the Celestron 14" optical tube assembly with a Paramount ME German equatorial mount at the Villanova University Observatory located on the campus near Philadelphia, PA. Observations were carried out with a SBIG ST7-XME detector thermoelectrically cooled to -25 degrees Celsius. Dark and flat field frames were collected at the end of each nightŠs observations. A standard error of $0.05 \mathrm{mag}$ was estimated from the signal-to-noise ratio and seeing conditions. Table A.3 (available online only) lists the magnitudes obtained at Villanova.

\subsubsection{Additional data}

We have also used published optical and near-infrared photometric data from Lorenzetti et al. (2007) (IJHK), Garcia et al. (2006) and Garcia \& Parsamian (2008) (V).

\subsection{Spitzer}

In addition to our observations of V1118 Ori in outburst (program ID 3716, PI: G. Stringfellow) and in post-outburst (program ID 41019, PI: M. Audard), V1118 Ori was serendipitously observed with the InfraRed Array Camera (IRAC; Fazio et al. 2004) by the Spitzer Space Telescope (Werner et al. 2004) in March 2004 and twice in October 2004 (program IDs 43 and 50, PI: G. Fazio). We show the IRAC images taken before the outburst in March 2004 centered on V1118 Ori

\footnotetext{
2 SMARTS, the Small and Medium Aperture Research Telescope Facility, is a consortium of universities and research institutions that operate the small telescopes at Cerro Tololo under contract with AURA.
}

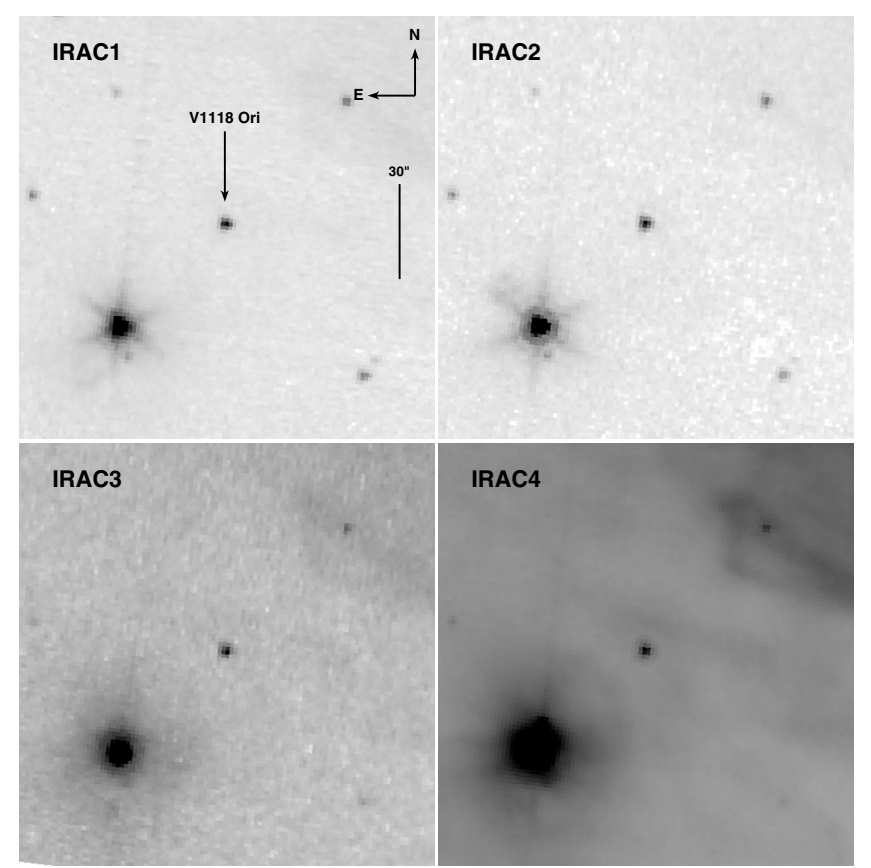

Fig. 1. Spitzer IRAC images centered on V1118 Ori, taken pre-outburst in March 2004. The images are shown with a linear scale from 0 to $200 \mathrm{MJy} \mathrm{sr}^{-1}$. The Herbig Ae star V372 Ori is seen near V1118 Ori.

(Fig. 1). We provide the IRAC fluxes for all observations in Table 2 (see also Lorenzetti et al. 2007 for the IRAC data of October 27, 2004). Details of the Spitzer IRAC data reduction are given in Appendix B.

V1118 Ori was also observed with the Multiband Imaging Photometer for Spitzer (MIPS; Rieke et al. 2004) at $24 \mu \mathrm{m}$ before the outburst, on March 20, 2004 (program ID 58, PI: G. Rieke). Again, Appendix B provides details of the data reduction. No MIPS photometry is available for V1118 Ori during or after the outburst. Our programs (3716 and 41019) included MIPS spectral energy distribution data $(R \approx 20)$ in the $70 \mu \mathrm{m}$ band; however, the on-time exposure $(180 \mathrm{~s})$ did not allow us to detect V1118 Ori, even during the outburst. Indeed, the background level (because of the diffuse emission in the Orion nebula) produced a much higher flux (on the order of $40 \mathrm{Jy}$ at $70 \mu \mathrm{m}$ ) than the expected signal from V1118 Ori (on the order $0.1 \mathrm{Jy}$ if extrapolating the SED at $70 \mu \mathrm{m}$, see Fig. 12).

Spitzer also observed V1118 Ori with the InfraRed Spectrograph (IRS; Houck et al. 2004) twice during the outburst (PID 3716) with the Short-Low (SL: 5.2-14.7 $\mu \mathrm{m}, R=$ $\lambda / \Delta \lambda \approx 64$ ), Short-High (SH: 9.9-19.6 $\mu \mathrm{m}, R=\lambda / \Delta \lambda \approx 600$ ), and Long-High (LH: 18.7-37.2 $\mu \mathrm{m}, R=\lambda / \Delta \lambda \approx 600$ ) modules (2005 February 18 and 2005 March 11). No background observations were taken with the high-resolution modules. In contrast, the post-outburst data (PID 41019; 2008 November 14) 


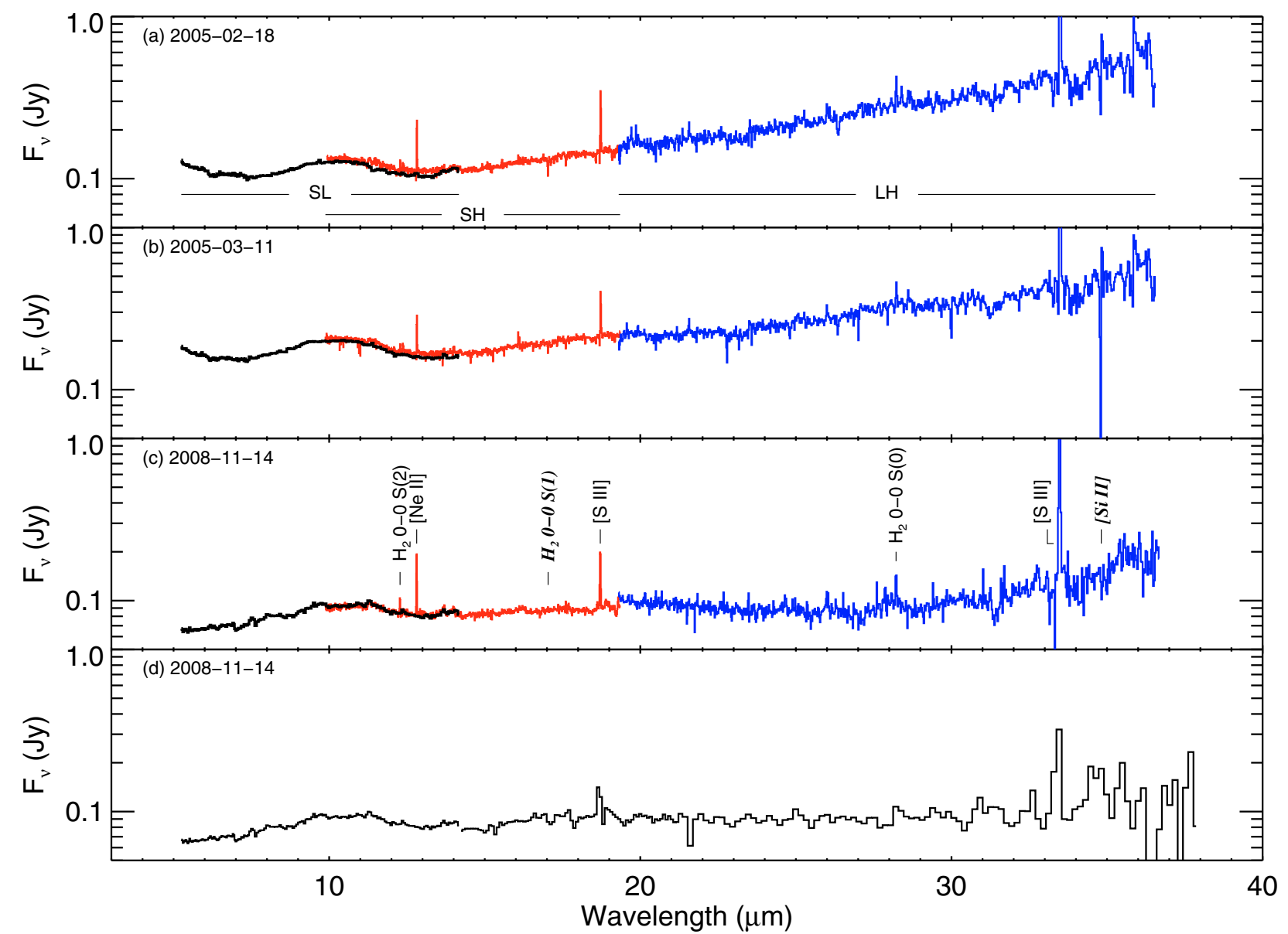

Fig. 2. Spitzer IRS background-subtracted spectra obtained near the peak of the outburst (panels a) and b)) and after the outburst (panels c) and d)). Panel d) shows the SL and LL module data. Panels a), b), and c) show the SL spectra as thick black lines, whereas the SH and LH spectra are shown as red and blue lines. Detected emission lines are labeled (while other lines well-subtracted by the background spectrum are shown in italics).

were taken with background spectra for the high-resolution modules and also included the Long-Low (LL: $14.0-38.0 \mu \mathrm{m}, R=$ $\lambda / \Delta \lambda \approx 64)$ module.

Figure 2 shows all IRS spectra after background subtraction. We describe in Appendix B the methodology we used to derive the background-subtracted spectra of V1118 Ori. We also discuss in the Appendix the validity of using the post-outburst background observation for our outburst IRS SH spectra.

In brief, the background is well-subtracted in the case of the continuum in the post-outburst, high-resolution spectrum, as the flux is consistent with the low-resolution spectrum. The continua in the outburst SH spectra are also accurate below $14 \mu \mathrm{m}$ since they are consistent with the low-resolution SL spectra. However, we consider the observed increase in continuum flux for $\lambda>14 \mu \mathrm{m}$ during the outburst to be unreliable, since we have no MIPS photometry or low-resolution spectra to confirm the increase. We also urge caution with regards to the detection of lines in the background-subtracted $\mathrm{SH}$ and LH spectra. The strong background line emission of the Orion nebula, and its inhomogeneity (see Fig. B.2) make the background subtraction difficult, although not impossible (e.g., a very strong PAH emission at $11.3 \mu \mathrm{m}$ is well-subtracted). The [S III] emission lines at 18.71 and $33.48 \mu \mathrm{m}$ are likely robust detections as their detection is consistent with the low-resolution SL and LL data. The [Si II] line is also generally well subtracted.

Interestingly, the rotational $\mathrm{H}_{2} \mathrm{~S}(0)$ line is detected during the outburst and in post-outburst. However the $\mathrm{S}(1)$ line is not detected (it is is even slightly oversubtracted in the outburst spectra), and the $\mathrm{S}(2)$ line is only detected in the post-outburst spectrum (a faint excess in the outburst spectra is present only in one bin, likely caused by slight differences in the wavelength scales of the source and background spectra). It is likely that the $\mathrm{H}_{2}$ emitting conditions vary spatially in the Orion nebula, and that the background spectrum did not accurately reflect the $\mathrm{H}_{2}$ background line fluxes near V1118 Ori, creating an excess in line emission observed in the post-outburst spectrum. Alternatively, if the $\mathrm{H}_{2}$ emission lines are real, at least in the post-outburst spectra, the physical conditions near V1118 Ori are such that the para (odd quantum number $J$ ) lines dominate the emission.

A similar case of difficult background line subtraction arises in the [Ne II] line at $12.81 \mu \mathrm{m}$ : its detection in the $\mathrm{SH}$ spectrum is also subject to caution, as it is not detected in the low-resolution spectra (although it should have been, see the discussion in the Appendix). In any case, we provide in Table 3 the tentative, measured line fluxes from the high-resolution module spectra, while Fig. 3 shows zoomed in regions of the IRS spectra of the detected lines.

\section{X-ray analysis}

\subsection{Light curves}

Figure 4 presents the X-ray light curves for the Chandra observations. The lower panels show the binned light curves, whereas 

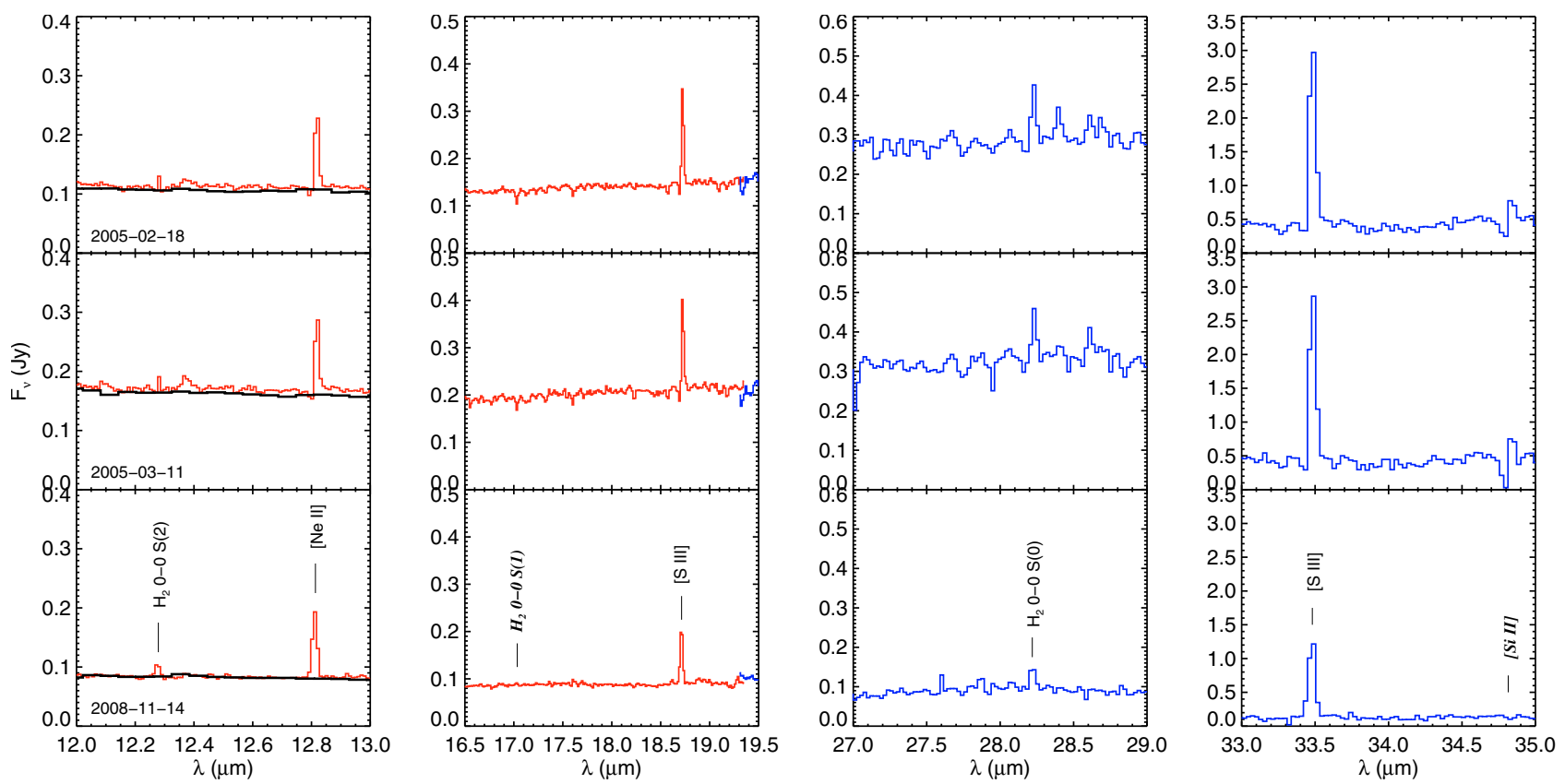

Fig. 3. Zoomed in regions of the Spitzer IRS background-subtracted spectra. The post-outburst spectra are shown in the bottom panel, while the top two panels are for the outburst spectra. In the leftmost panels, we show both the high-resolution SH (thin, red) and the low-resolution SL (thick, black) data. Detected emission lines are labeled in the bottom panels (while other lines well-subtracted by the background spectrum are shown in italics). Observation dates applicable to each horizontal set of spectra are listed in the left panels.

Table 3. Tentative Spitzer IRS line fluxes derived from the SH and LH spectra.

\begin{tabular}{lrrrr}
\hline \hline Line & $\lambda(\mu \mathrm{m})$ & \multicolumn{3}{c}{ Flux $\left(10^{-18} \mathrm{~W} \mathrm{~m}^{-2}\right)$} \\
& & Feb. 2005 & Mar. 2005 & Nov. 2008 \\
\hline $\mathrm{H}_{2} 0-0 S(2)$ & 12.2786 & & & $7.8 \pm 0.8$ \\
{$[\mathrm{Ne} I \mathrm{II}]{ }^{2} P_{1 / 2}-{ }^{2} P_{3 / 2}$} & 12.8136 & $41.1 \pm 0.7$ & $39.7 \pm 1.1$ & $42.7 \pm 0.9$ \\
{$\left[\mathrm{~S} \mathrm{III}^{3} P_{2}-{ }^{3} P_{1}\right.$} & 18.7130 & $46.5 \pm 1.4$ & $42.5 \pm 1.5$ & $34.6 \pm 1.2$ \\
$\mathrm{H}_{2} 0-0 S_{0}(0)$ & 28.2188 & $15.2 \pm 2.2$ & $18.9 \pm 3.4$ & $9.5 \pm 1.6$ \\
{$[\mathrm{~S}$ III $]{ }^{3} P_{1}-{ }^{3} P_{0}$} & 33.4810 & $463.3 \pm 9.6$ & $374.4 \pm 7.9$ & $183.4 \pm 4.7$ \\
\hline
\end{tabular}

the upper panels show the photon CCD energy as a function of time. Except for Sep. 2002, the background is not subtracted in the light curves and not shown in the upper panels, since it contributed less than 1 count to the Chandra total (source + background) number of counts over the entire observing duration. Since V1118 Ori was observed serendipitously in the Sep. 2002 observation and was placed at the edge of the ACIS-I camera, the background contributed more significantly; therefore, the light curve shown in the bottom panel is background-subtracted, and the CCD energy vs time background events are shown together with the CCD energy vs. time total events (for clarity, every ninth background event was plotted, which corresponds approximately to the area-scaled background contribution).

While small-scale variability is visible in the Chandra light curves, no strong flares were detected during the observations, although a significant increase in the count rate in the last $10 \mathrm{ks}$ of the January 2006 observation may be attributed to a moderate flare. The average count rates are 3.14, 2.31, 1.10, 0.39, $1.23,1.23$, and $1.09 \mathrm{ct} \mathrm{ks}^{-1}(0.2-4.0 \mathrm{keV}$, except for Sep. 2002: 0.2-6.0 keV) for the Sep. 2002, Jan. 2005, Jan.-Jul. 2006, and Dec. 2007 observations, respectively. While the effective areas in all pointed observations are similar, the Sep. 2002 effective area was about $40-50 \%$ lower (because of the off-axis position of V1118 Ori). Therefore, to compare the above Sep. 2002 count rate with the later Chandra count rates, one needs to multiply by a factor of about 2, i.e., $6.00 \mathrm{ct} \mathrm{ks}^{-1}$. Clearly, assuming only a change in emission measure, the X-ray flux dropped by a factor of 4-6, and even 15 in Feb. 2006, during the 2005 outburst compared to 2002. It is important to emphasize that the serendipitous observation did not show evidence of strong flaring, and V1118 Ori was likely caught in "quiescence".

Figure 5 presents the X-ray light curves for the $X M M-N e w t o n$ observations. In comparison with the Chandra data, the XMM-Newton data were heavily impacted by background radiation. The (scaled) background contributions were generally similar to - or even higher than - the net source contribution. In particular, during the March 2005 observation, the background completely overwhelmed the EPIC pn data which could not be used. As in the Chandra observations, no obvious large flare was observed during the XMM-Newton exposures, However, in March 2006, V1118 Ori showed a significant increase in X-ray flux in the last $35-40 \mathrm{ks}$ of the observation, probably due to flares or the onset of an active region. This increase is observed in all EPIC cameras, comforting us that this behavior is not caused by an increase in background flux that was improperly subtracted in the EPIC pn data.

The light curves in Figs. 4 and 5 suggest that the spectral fits represent snapshots of the thermal emission measure distribution of V1118 Ori's corona observed serendipitously before the 2005 outburst, and over the course of the optical/infrared outburst.

\subsection{X-ray and optical/near-infrared flux correlations}

Figure 6 shows the long-term light curve of V1118 Ori in the $\mathrm{X}$-rays, optical, and infrared, from its pre-outburst detection in September 2002, through its outburst in 2005 and 2006 to the post-outburst phase at the end of December 2007. We have looked into correlations between the X-ray and optical and infrared photometry. Since we obtained few sequential optical and infrared photometry points every night of our campaign, it was 

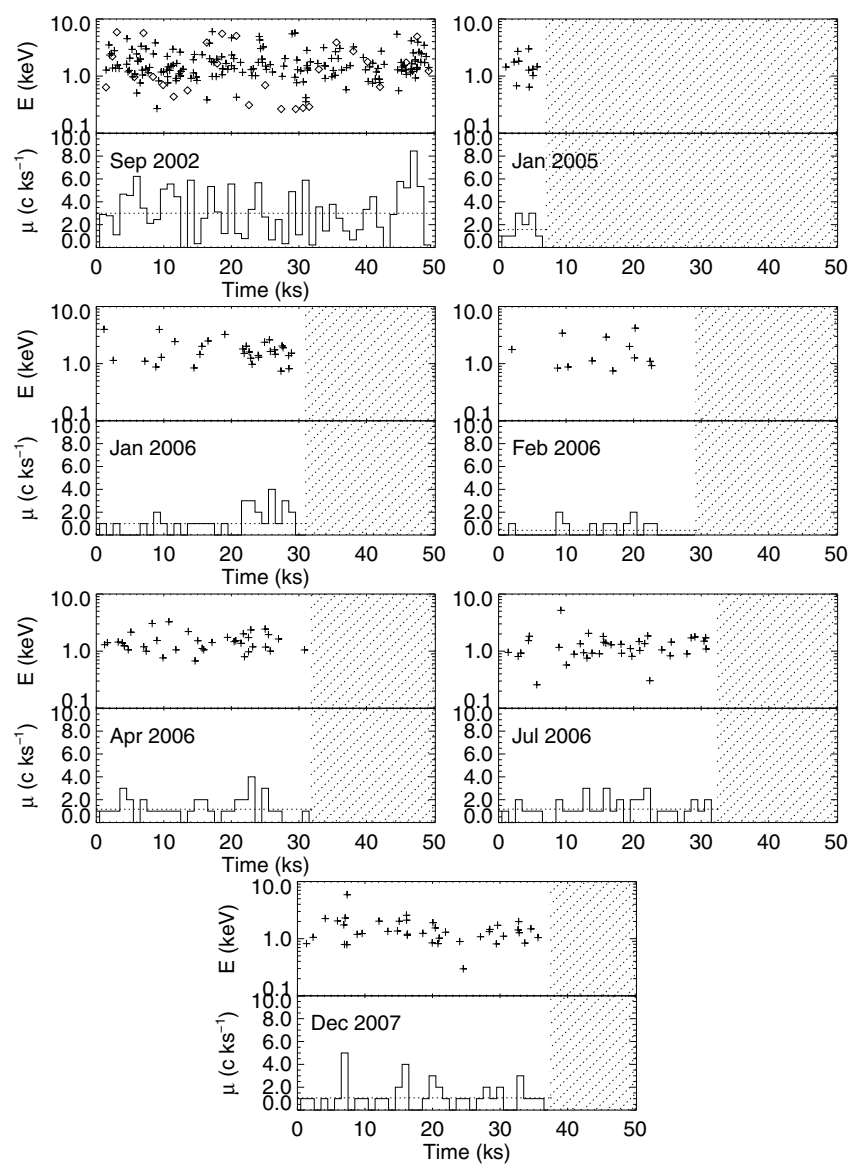

Fig. 4. Light curves for the Chandra observations (0.4-6.0 keV range). The upper panels show the CCD energy of the events as a function of time after the start of the observations, whereas the lower panels show count rate light curves $(\mu)$ with a bin size of $1 \mathrm{ks}$. The average count rates are also shown as horizontal dotted lines. The same time span, equal to that of the Sep. 2002 observation, is covered in each panel. Since the other observations were shorter than $50 \mathrm{ks}$, the rest of the time span is marked by hashed regions. See text for a detailed description.

not possible to determine short-term variations to compare with the X-ray light curves. Nevertheless, to quantify further the correlation between the optical and near-infrared flux densities with the X-ray flux, we have calculated average optical and nearinfrared flux densities within \pm 20 days of the 6 X-ray observations between January 2005 and February 2006 (we did not include the March 2006 observation, as this one might have been contaminated by a flare). The optical and near-infrared flux densities are well-correlated with the X-ray fluxes measured at Earth (Fig. 7) as indicated by Pearson's correlation coefficients, which are in the range of $\rho=0.8-0.95$. The sparsity of X-ray data points does not allow the measurement of any clear delay between the X-ray and optical/near-infrared flux densities. We have also performed cross-correlation studies with the optical and near-infrared data (including only the data points for which we had simultaneous measurements). The onset of the outburst is unclear in most light curves (except perhaps in the $V$ band), but the end of the outburst occurs at a similar epoch for all bands (around MJD 53860). The shapes of the light curves also differ: the fluxes vary most at short wavelengths ( $B V R I)$, while the near-infrared fluxes display shallower variations and they reach their peak some time later (e.g., $K$ peaks around MJD 53470 while $V$ peaks around MJD 53433). The overall shapes of the light curves suggest that the mechanism dominating in the
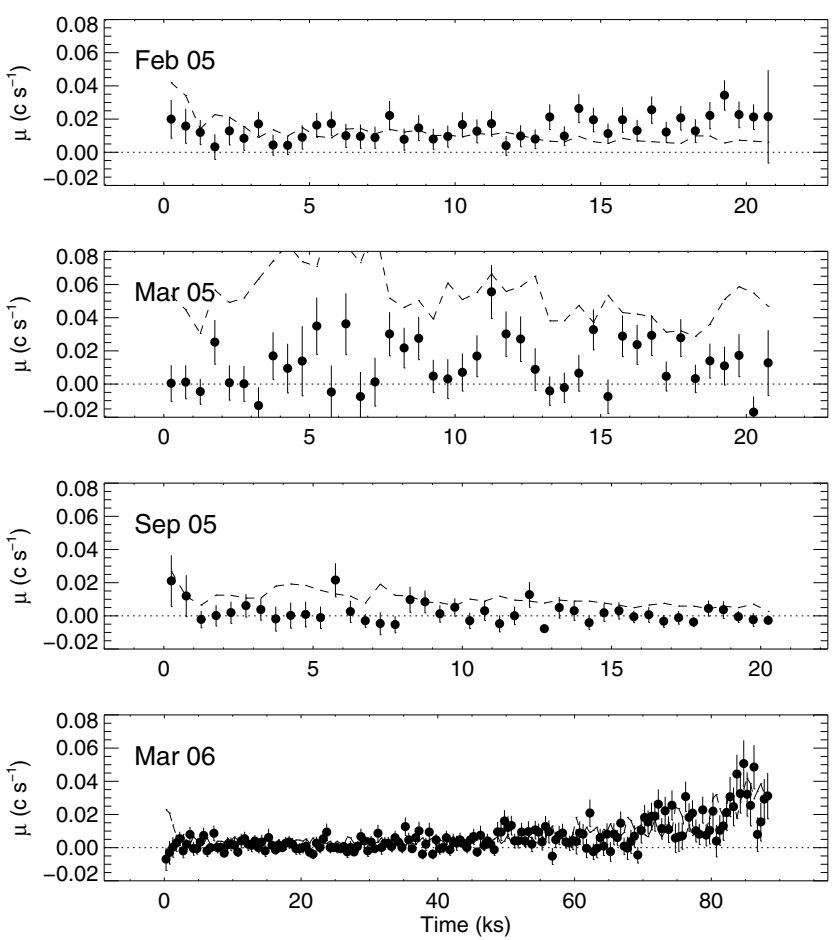

Fig. 5. Background-subtracted count rate light curves $(\mu)$ for the XMM-Newton observations, with bin sizes of $500 \mathrm{~s}$ and in the 0.4-6.0 keV range. The EPIC pn data are shown, except in March 2005 for which we summed the data of both MOS detectors. The (scaled) background light curves are shown as dashed curves.

optical and near-infrared bands may be the same (e.g., due to a hot spot), although the different peak times suggest contamination of another mechanism longward of $1 \mu \mathrm{m}$, probably disk thermal emission (see below).

\subsection{Spectral fits}

Audard et al. (2005b) presented spectral fits of the V1118 Ori data taken through March 2005. Lorenzetti et al. (2006) repeated the analysis for the February 2005 data and added the September 2005 data, noting a decrease in the X-ray flux of V1118 Ori at that time compared to earlier in the outburst. For this paper, we have reprocessed the 2005 data with the latest calibration (see Sect. 3).

We used XSPEC 11 (Arnaud 1996) to fit the Chandra ACIS and the XMM-Newton EPIC spectra for each epoch. For February 2005 and March 2006, we fitted the EPIC pn, MOS1, and MOS2 spectra simultaneously, while we used the EPIC MOS only for March 2005, and the EPIC pn only for September 2005 (no MOS data were available). In general, we used a 1-T collisional ionization equilibrium (CIE) model (apec; Smith et al. 2001) with a photoelectric absorption model, except for the deep March 2006 observation for which the signal-to-noise was large enough to use a 2-T CIE model. We have fitted the plasma metallicity in the high signal-to-noise ratio spectra of February 2005 and used the best-fit value, $Z=0.17 Z_{\odot}$, for the other epochs. The coronal abundances are relative to the solar photospheric standard set of Grevesse \& Sauval (1998). The best-fit metallicity is in line with the values measured for $\mathrm{Fe}$ in the coronae of young stars in Orion (Table 3 in Maggio et al. 2007). Since the spectral fits of the February and March 2005 spectra are similar to those reported in Audard et al. (2005b), 


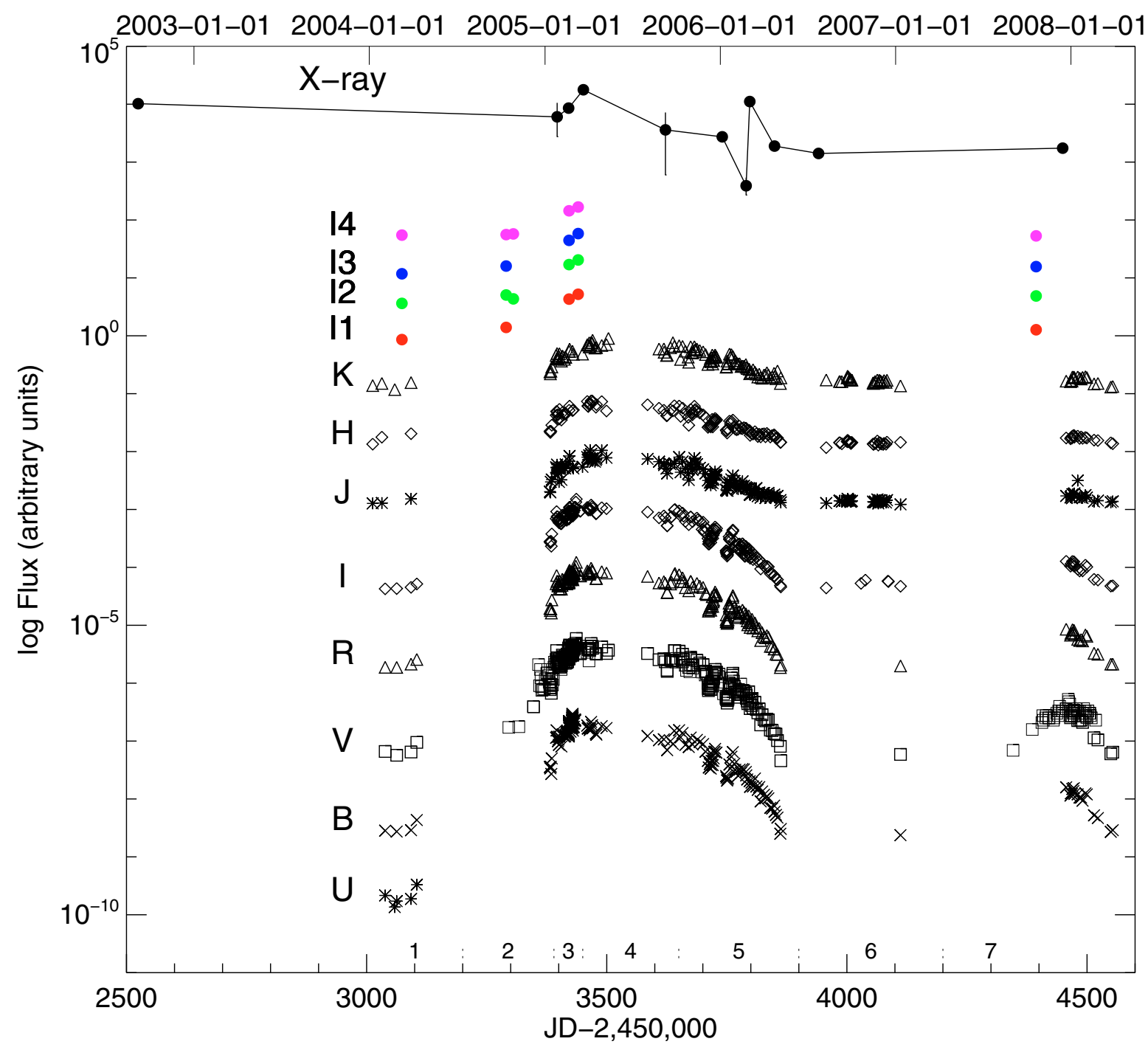

Fig. 6. Optical (UBVRI), near-infrared $(J H K)$, mid-infrared (IRAC bands at 3.6, 4.5, 5.8, $8.0 \mu \mathrm{m}$ ), and X-ray light curves versus Julian date. Arbitrary scales were applied to help visualize the light curves. See text for the definition of the time intervals labeled at the bottom. Data from the SMARTS and Villanova campaigns, from Lorenzetti et al. (2007), Garcia et al. (2006) and Garcia \& Parsamian (2008) are included.

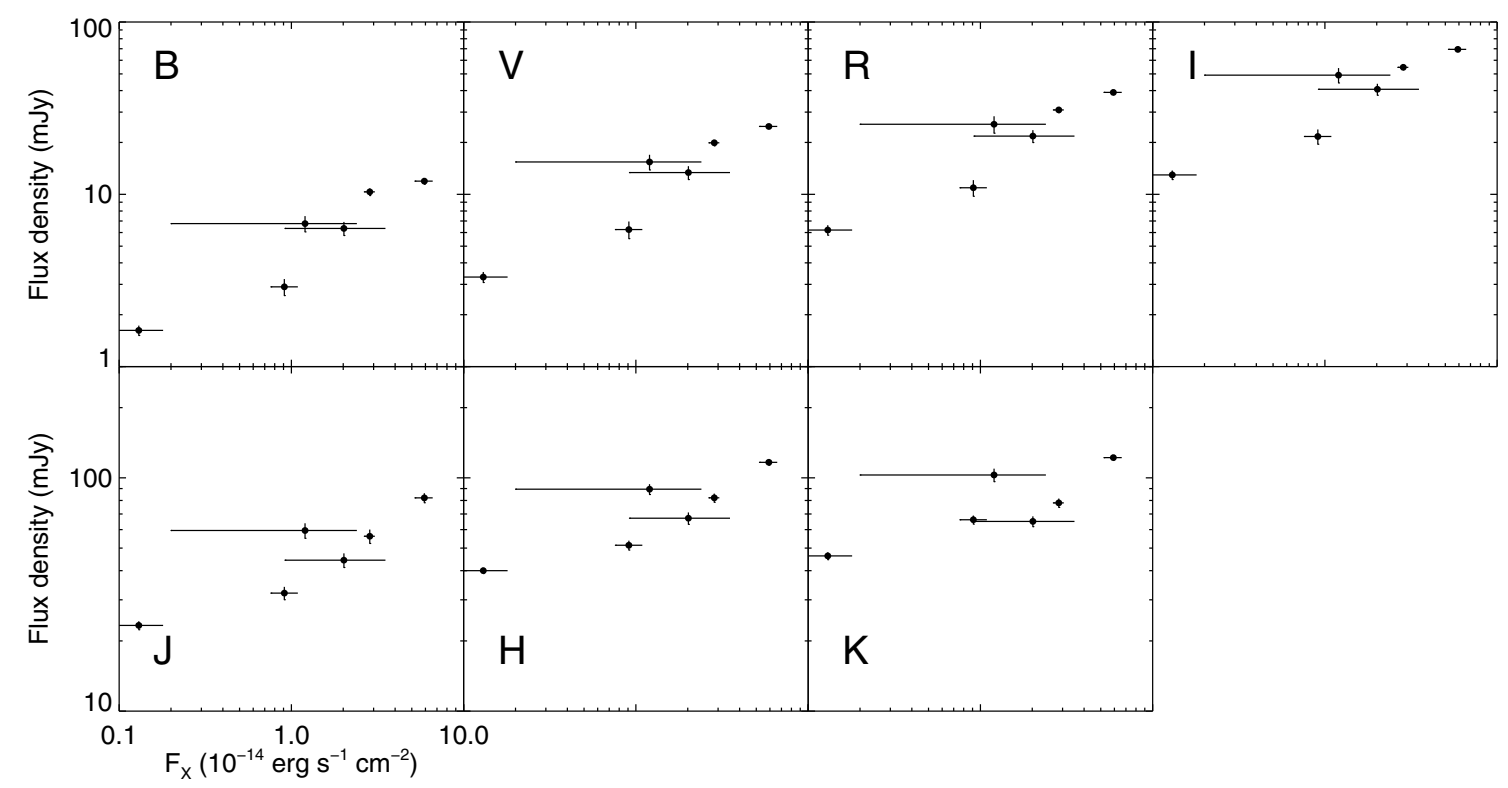

Fig. 7. Optical and near-infrared flux densities (averaged within \pm 20 days of the X-ray observation) as a function of the observed X-ray flux during the outburst (Jan. 2005 to Feb. 2006). Notice the different flux axis scales for the optical and near-infrared bands. 
Table 4. Spectral fits and quantile properties.

\begin{tabular}{|c|c|c|c|c|c|c|}
\hline Parameter & Sep. 2002 & & Jan. 2005 & Feb. 2005 & Mar. 2005 & Sep. 2005 \\
\hline Satellite & Chandra & & Chandra & XMM-Newton & XMM-Newton & XMM-Newton \\
\hline \multicolumn{7}{|l|}{ Spectral analysis: } \\
\hline Net counts ${ }^{a}$ & 150.9 & & 10.8 & 285.5/105.4/103.2 & $(47.1) / 190.4 / 108.0$ & (12.4) \\
\hline Scaled background counts ${ }^{a}$ & 27.1 & & 0.2 & $224.5 / 56.6 / 52.8$ & $(698.9) / 325.6 / 331.0$ & $(227.6)$ \\
\hline Exposure $(\mathrm{ks})^{a}$ & 47.85 & & 4.66 & $18.34 / 21.87 / 21.91$ & $(7.83) / 19.96 / 19.22$ & (13.03) \\
\hline$N_{\mathrm{H}}\left(10^{21} \mathrm{~cm}^{-2}\right)$ & $2.7_{-09}^{+1.2}$ & & $1.4_{-14}^{+3.6}$ & $4.3_{-1.1}^{+1.3}$ & $1.4_{-05}^{+0.8}$ & \\
\hline$T(\mathrm{MK})$ & $25.1_{-4.8}^{-6.9}$ & & $46_{-28}^{+\infty .4}$ & $7.7_{-0.8}^{-1.1 .3}$ & $15.9_{-2.0}^{+3.5}$ & \\
\hline $\operatorname{EM}\left(10^{53} \mathrm{~cm}^{-3}\right)$ & $1.0_{-0.2}^{+0.3}$ & & $0.4_{-0.1}^{-28.4}$ & $2.9_{-1.0}^{+1.8}$ & $2.1_{-0.2}^{-0.0}$ & \\
\hline$Z / Z_{\odot}$ & $:=0.17$ & & $:=0.17$ & $0.17_{-0.06}^{+0.20}$ & $:=0.17$ & \\
\hline$F_{\mathrm{X}}\left(10^{-14} \mathrm{erg} \mathrm{cm}^{-2} \mathrm{~s}^{-1}\right)$ & $3.4_{-0.3}^{+0.3}$ & & $2.0_{-1.1}^{+1.5}$ & $2.9_{-0.2}^{+0.20}$ & $5.9_{-0.7}^{+0.7}$ & $1.2_{-10}^{+1.2 b}$ \\
\hline$L_{\mathrm{X}}\left(10^{30} \mathrm{erg} \mathrm{s}^{-1}\right)$ & $1.2_{-0.1}^{-0.3}$ & & $0.5_{-0.3}^{-1.1}$ & $2.5_{-0.2}^{+0.2}$ & $2.1_{-0.2}^{-0.7}$ & $0.04-0.12^{b}$ \\
\hline \multicolumn{7}{|l|}{ Quantile analysis: } \\
\hline Energy range & $0.2-6.0$ & & $0.2-4.0$ & $0.2-4.0$ & $0.2-4.0$ & $0.2-4.0$ \\
\hline Net counts ${ }^{c}$ & 150.44 & & 10.79 & 286.45 & 196.34 & 9.75 \\
\hline Scaled background counts ${ }^{c}$ & 26.56 & & 0.21 & 222.55 & 425.66 & 228.25 \\
\hline$Q_{25 \%}$ & $0.17 \pm 0.01$ & & $0.24 \pm 0.07$ & $0.15 \pm 0.01$ & $0.15 \pm 0.02$ & $0.0 \pm 0.5$ \\
\hline$Q_{50 \%}$ & $0.22 \pm 0.01$ & & $0.33 \pm 0.07$ & $0.20 \pm 0.01$ & $0.25 \pm 0.02$ & $0.3 \pm 1.5$ \\
\hline$Q_{75 \%}$ & $0.31 \pm 0.03$ & & $0.42 \pm 0.14$ & $0.29 \pm 0.01$ & $0.34 \pm 0.03$ & $0.9 \pm 1.3$ \\
\hline$E_{50 \%}(\mathrm{keV})$ & $1.45 \pm 0.06$ & & $1.44 \pm 0.25$ & $0.95 \pm 0.04$ & $1.14 \pm 0.07$ & $1.2 \pm 4.0$ \\
\hline$x=\log \left[Q_{50 \%} /\left(1-Q_{50 \%}\right)\right]$ & $-0.56_{-0.03}^{+0.03}$ & & $-0.31_{-0.14}^{+0.12}$ & $-0.61_{-0.03}^{+0.03}$ & $-0.48_{-0.04}^{+0.04}$ & $-0.4_{-\infty}^{+\infty}$ \\
\hline$y=3 \times Q_{25 \%} / Q_{75 \%}$ & $1.63_{-0.13}^{+0.13}$ & & $1.70_{-0.61}^{+0.61^{4}}$ & $1.54_{-0.07}^{+0.07}$ & $1.33_{-0.19}^{+0.19}$ & $0.05_{-0.05}^{+1.42}$ \\
\hline$T_{x y}(\mathrm{MK})$ & $\approx 20$ & & $\approx 12$ & $\approx 12$ & $\approx 20$ & \\
\hline \multirow[t]{2}{*}{$N_{\mathrm{H}, x y}\left(10^{21} \mathrm{~cm}^{-2}\right)$} & $\approx 3$ & & $\approx 8$ & $\approx 1$ & $\approx 0.8$ & \\
\hline & Jan. 2006 & Feb. 2006 & Mar. 2006 & Apr. 2006 & Jul. 2006 & Dec. 2007 \\
\hline Satellite & Chandra & Chandra & XMM-Newton & Chandra & Chandra & Chandra \\
\hline \multicolumn{7}{|l|}{ Spectral analysis: } \\
\hline Net counts ${ }^{a}$ & 30.5 & 10.5 & $623.4 / 183.8 / 232.4$ & 36.4 & 36.3 & 37.9 \\
\hline Scaled background counts ${ }^{a}$ & 0.5 & 0.5 & $998.6 / 215.2 / 209.6$ & 0.6 & 0.7 & 1.1 \\
\hline Exposure $(\mathrm{ks})^{a}$ & 27.82 & 27.11 & $78.38 / 90.90 / 90.95$ & 29.57 & 29.57 & 34.73 \\
\hline$N_{\mathrm{H}}\left(10^{21} \mathrm{~cm}^{-2}\right)$ & $3.1_{-1.9}^{+2.4}$ & $3.6_{-3.1}^{+4.3}$ & $4.2_{-1.1}^{+0.3}$ & $4.9_{-20}^{+5.2}$ & $7.3_{-2.4}^{+2.2}$ & $2.5_{-10}^{+1.6}$ \\
\hline$T(\mathrm{MK})$ & $46.8_{-260}^{+\infty .9}$ & $8.7_{-35}^{-9.1}$ & $11.3_{-28}^{+3.5} / 89_{-43}^{+32}$ & $15.9_{-70}^{+8.6}$ & $6.5_{-2.1}^{+2.4}$ & $19.9^{+1.0} .4$ \\
\hline $\operatorname{EM}\left(10^{53} \mathrm{~cm}^{-3}\right)$ & $0.18_{-0.05}^{+0.0}$ & $0.10_{-0.34}^{+0.34}$ & $0.22_{-0.13}^{+0.12} / 0.51_{-0.07}^{+0.08}$ & $0.35_{-0.11}^{+0.03}$ & $1.0_{-10}^{+2.1}$ & $0.20_{-0.05}^{+0.78}$ \\
\hline$Z / Z_{\odot}$ & $:=0.17$ & $:=0.17$ & $:=0.17$ & $:=0.17$ & $:=0.17$ & $:=0.17$ \\
\hline$F_{\mathrm{X}}\left(10^{-14} \mathrm{erg} \mathrm{cm}^{-2} \mathrm{~s}^{-1}\right)$ & $0.91_{-0.15}^{+0.18}$ & $0.13_{-0.04}^{+0.05}$ & $3.7_{-0.2}^{+0.2}$ & $0.63_{-0.10}^{+0.11}$ & $0.47_{-0.07}^{+0.08}$ & $0.58_{-0.09}^{+0.10}$ \\
\hline$L_{\mathrm{X}}\left(10^{30} \mathrm{erg} \mathrm{s}^{-1}\right)$ & $0.27_{-0.05}^{+0.05}$ & $0.10_{-0.03}^{+0.04}$ & $1.16_{-0.07}^{+0.27}$ & $0.36_{-0.06}^{+0.06}$ & $0.9_{-0.1}^{-0.07}$ & $0.20_{-0.03}^{+0.09}$ \\
\hline \multicolumn{7}{|l|}{ Quantile analysis: } \\
\hline Energy range & $0.2-4.0$ & $0.2-4.0$ & $0.3-10.0$ & $0.2-4.0$ & $0.2-4.0$ & $0.2-4.0$ \\
\hline Net counts $^{c}$ & 30.48 & 10.53 & 626.9 & 36.37 & 36.32 & 37.91 \\
\hline Scaled background counts ${ }^{c}$ & 0.52 & 0.47 & 985.1 & 0.63 & 0.68 & 1.09 \\
\hline$Q_{25 \%}$ & $0.28 \pm 0.04$ & $0.18 \pm 0.03$ & $0.081 \pm 0.010$ & $0.23 \pm 0.02$ & $0.18 \pm 0.01$ & $0.22 \pm 0.03$ \\
\hline$Q_{50 \%}$ & $0.35 \pm 0.04$ & $0.24 \pm 0.09$ & $0.145 \pm 0.006$ & $0.31 \pm 0.03$ & $0.24 \pm 0.04$ & $0.28 \pm 0.02$ \\
\hline$Q_{75 \%}$ & $0.48 \pm 0.08$ & $0.46 \pm 0.19$ & $0.255 \pm 0.019$ & $0.40 \pm 0.06$ & $0.34 \pm 0.03$ & $0.40 \pm 0.06$ \\
\hline$E_{50 \%}(\mathrm{keV})$ & $1.52 \pm 0.15$ & $1.12 \pm 0.35$ & $1.709 \pm 0.06$ & $1.39 \pm 0.09$ & $1.11 \pm 0.14$ & $1.28 \pm 0.09$ \\
\hline$x=\log \left[Q_{50 \%} /\left(1-Q_{50 \%}\right)\right]$ & $-0.27_{-0.08}^{+0.07}$ & $-0.50_{-0.26}^{+0.20}$ & $-0.77_{-0.02}^{+0.02}$ & $-0.34_{-0.05}^{+0.05}$ & $-0.50_{-0.09}^{+0.08}$ & $-0.40_{-0.05}^{+0.05}$ \\
\hline$y=3 \times Q_{25 \%} / Q_{75 \%}$ & $\begin{array}{l}.00 .08 \\
1.74_{-0.29}^{+0.29}\end{array}$ & $1.19_{-0.42}^{+0.026}$ & $0.95_{-0.11}^{-0.0 .02}$ & $1.68_{-0.22}^{+0.22}$ & $1.65_{-0.15}^{+0.19^{9}}$ & $1.63_{-0.25}^{+0.25}$ \\
\hline$T_{x y}(\mathrm{MK})$ & $\approx 13^{-29}$ & $\approx 35^{-0.2}$ & $\approx 90$ & $\approx 10^{-0.22}$ & $\approx 15$ & $\approx 16$ \\
\hline$N_{\mathrm{H}, x y}\left(10^{21} \mathrm{~cm}^{-2}\right)$ & $\approx 10$ & $\approx 0.2$ & $\approx 2.5$ & $\approx 9.0$ & $\approx 1.5$ & $\approx 3.5$ \\
\hline
\end{tabular}

Notes. The uncertainties are based on $68 \%$ Bayesian confidence ranges. X-ray luminosity and absorbed X-ray flux in the $0.1-10 \mathrm{keV}$ range, assuming $d=400 \mathrm{pc}$.

(a) For XMM-Newton observations, values refer to the EPIC pn, MOS1, and MOS2, respectively (pn only for September 2005). The values in parentheses refer to detectors not used in the spectral fits; ${ }^{(b)}$ estimates based on models for February 2005 and March 2006; ${ }^{(c)}$ based on pn data in general, except for March 2005 for which MOS1 data only were used (similar values and results were obtained for MOS2 data).

we provide in this paper their best-fit values (but adapt the emission measure and luminosities to the adopted distance of $400 \mathrm{pc}$ ). The September 2005 data are heavily contaminated by the high background level during the observation. Contrary to Lorenzetti et al. (2006), we preferred not to provide spectral fits for this observation; however, we provide estimates for the observed X-ray flux at Earth and absorption-corrected X-ray luminosity, based on the previous February 2005 and the subsequent March 2006
XMM-Newton observations. The results of our spectral fits are given in Table 4.

\subsection{Quantiles}

In view of the low observed count rates with XMM-Newton and Chandra during the outburst, we have explored an alternative method to determine spectral properties for low-count 
A\&A 511, A63 (2010)
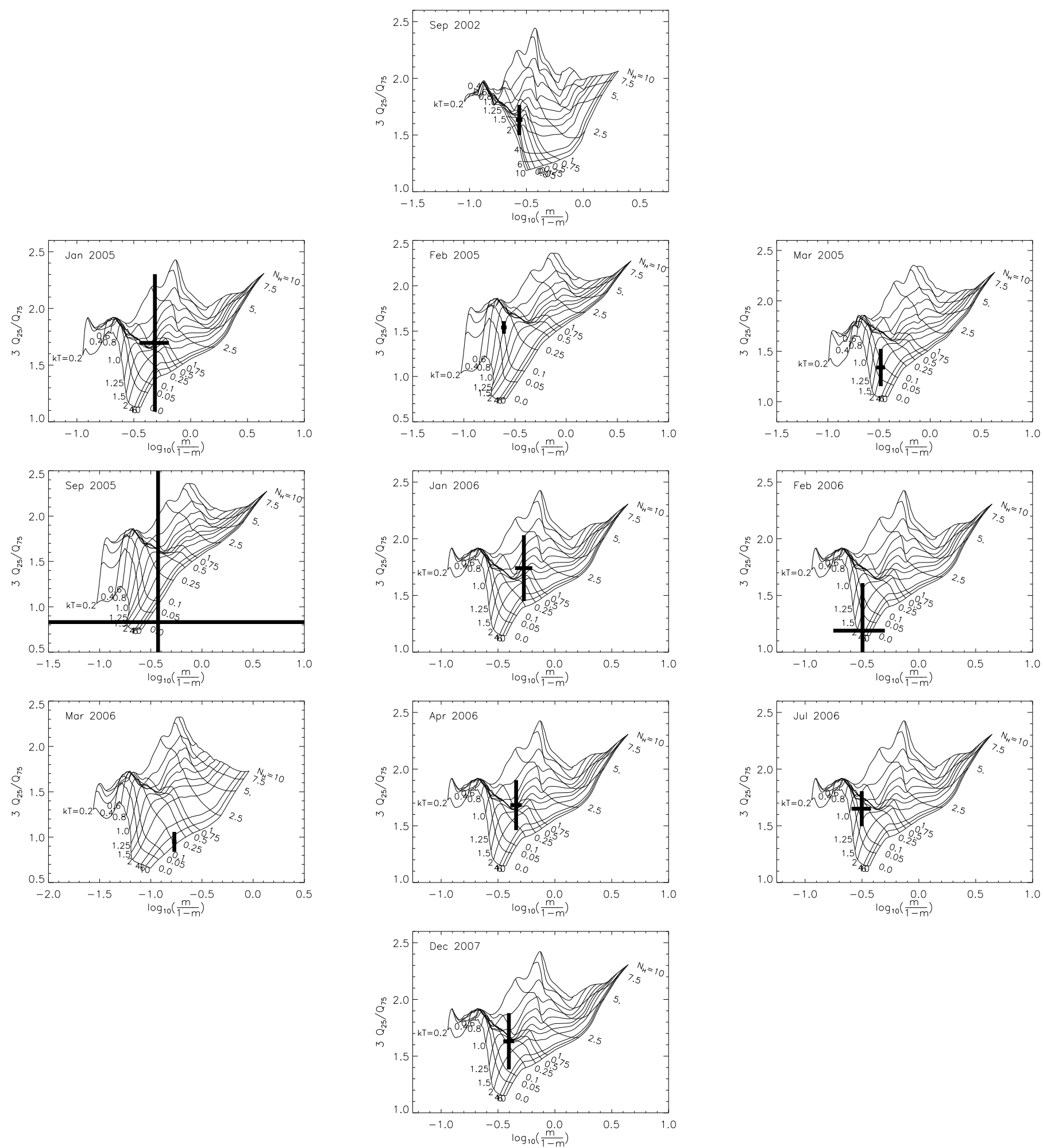

Fig. 8. Color-color diagrams based on the $Q_{50 \%}(=m), Q_{25 \%}$, and $Q_{75 \%}$ quantiles. The grid is in plasma temperature $(k T$ in keV) and hydrogen column density $\left(N_{\mathrm{H}}\right.$ in $\left.10^{22} \mathrm{~cm}^{-2}\right)$. The serendipitous, pre-outburst observation of September 2002 is shown at the top, the post-outburst version of December 2007 is shown at the bottom. The 9 outburst observations in between are ordered in time from left to right and top to bottom, with January 2005 at the top left and July 2006 at the bottom right.

X-ray spectra and have used the quantile analysis presented by Hong et al. (2004) ${ }^{3}$. This type of analysis was, in particular, successfully used by Grosso et al. (2005) for their analysis of the

3 The code is available at http://hea-www.harvard.edu/ ChaMPlane/quantile. We used version 1.7. spectral properties of V1647 Ori (see also Skinner et al. 2009b). In brief, this method makes direct use of the event energy values, and determines spectral properties based on quantiles of the total number of counts (e.g., median 50\% quantile, and quartiles, $25 \%$ and $75 \%$ ), instead of spectral energy bins. Such quantiles are 
then used as indicators for the X-ray color of the source. In particular, we make use of a diagram with the $x=\log _{10}(m /(1-m))$ index in the $x$-axis, where $m$ corresponds to the median quantile $Q_{50}$, and the index $y=3 \times Q_{25} / Q_{75}$ in the $y$-axis. Hong et al. (2004) define the $\alpha \%$ quantile, $Q_{\alpha}$, as

$Q_{\alpha}=\frac{E_{\alpha \%}-E_{\mathrm{lo}}}{E_{\text {up }}-E_{\mathrm{lo}}}$,

where $E_{\text {up }}$ and $E_{\mathrm{lo}}$ are the lower and upper boundaries of the used energy band, and $E_{\alpha \%}$ is the energy below which the net counts is $\alpha \%$ of the total number of counts. This method is able to take the distribution of background event energies into account. We refer the reader to Hong et al. (2004) for more details.

The total (source + background) and background extraction regions used in the quantile analysis were the same as those used for the spectral fits. The energy bands used to determine the extraction of events are reported in Table 4 together with the $25 \%, 50 \%$, and $75 \%$ quantiles, the $E_{50 \%}$ energy, and the $x$ and $y$ indices. We also provide estimated values for the hydrogen column density and plasma temperature derived from the $x$ and $y$ indices. Finally, Fig. 8 shows the color-color diagrams for all observations.

There exists a certain degree of degeneracy in the $x$ and $y$ indices (e.g., Grosso et al. 2005): indeed, the same color indices can sometimes be described by different combinations of column density $N_{\mathrm{H}}$ and plasma temperature $T$. However, these degeneracies are limited to certain sets of $T$ and $N_{\mathrm{H}}$ : for example, in the September 2002 observation, color indices of $x=-0.8$ and $y=$ 1.8 can represent any plasma temperature from about $k T \approx 0.2$ to about $k T \approx 0.8 \mathrm{keV}$ and $N_{\mathrm{H}}$ can range from 0 to $0.75 \times 10^{22} \mathrm{~cm}^{-2}$. At higher temperatures, the degeneracy breaks down, and the observed $x / y$ values of V1118 Ori can be better constrained to $k T \approx 1.2-2.5 \mathrm{keV}$ and $N_{\mathrm{H}} \approx(1-8) \times 10^{21} \mathrm{~cm}^{-2}$. The degeneracies differ slightly depending on the response matrix generated in the observation. For example, with our Chandra monitoring data, the degeneracies occur around $(x, y)=(-0.7,1.85)$ and at $(-0.4,1.65)$. The first set again indicate a degeneracy for $k T<0.8 \mathrm{keV}$ and $N_{\mathrm{H}}<1 \times 10^{22} \mathrm{~cm}^{-2}$. The second set shows that the plasma properties cannot be disentangled for temperatures between 0.8 and $1.5 \mathrm{keV}$ for a column density in the range $(3-8) \times 10^{21} \mathrm{~cm}^{-2}$.

In contrast to the temperatures obtained from spectral fits, the temperature derived from the color indices are more stable and vary little from $10-20 \mathrm{MK}$, except in February 2006 $\left(T_{x y} \approx 35 \mathrm{MK}\right)$ and March $2006\left(T_{x y} \approx 90 \mathrm{MK}\right)$. In the case of March 2006, the quantile temperature is in fact close to the second temperature component (which also has the higher emission measure) obtained with the 2- $T$ fit. In the case of February 2006, the low number of counts did not allow a good constraint on the temperature. There also appears to be some kind of degeneracy: while the spectral fit gives a low plasma temperature and "high" column density, the quantile analysis gives the inverse. It remains unclear which result is more robust, but the quantile diagram does not show evidence of a degeneracy. It is then likely that the spectral fit reached a local minimum at a low temperature and could not find a combination of high plasma temperature and low $N_{\mathrm{H}}$. The February 2005 quantile analysis and spectral fit both confirm that the temperature of the coronal plasma was cooler than in September 2002 and March 2005, as initially reported in Audard et al. (2005b). The error bars of the color indices in January 2005 do not allow us to constrain the temperature of the coronal plasma, while the spectral fit with XSPEC indicates no evidence of cool plasma at the start of the outburst.

\subsection{X-ray parameter correlations}

We have attempted to search for correlations between X-ray properties $\left(N_{\mathrm{H}}, T, N_{\mathrm{H}, x y}, T_{x y}\right.$, EM, $\left.L_{\mathrm{X}}, E_{50 \%}\right)$, but found generally little or no correlation based on the Pearson correlation coefficient, $\rho$ (absolute values typically below 0.5 ). One exception was a mild correlation between the average median photon energy, $E_{50 \%}$, and the absorbing column density derived from the quantile analysis, $N_{\mathrm{H}, x y}$, which showed $\rho=0.57$. This is expected, as larger column densities will absorb low-energy photons. However, we emphasize that we have only used the estimated $N_{\mathrm{H}, x y}$ based on the location of the $x y$ values in the quantile color-color diagrams, and that we have not taken into account the (relatively) large range of column densities covered by the quantile errors (typically, $1-5 \times 10^{21} \mathrm{~cm}^{-2}$, similar to values derived from spectral fitting). Another exception was a strong correlation between the emission measure and the X-ray luminosity, with $\rho=0.96$. Together with the above lack of correlation between the plasma temperature and $L_{\mathrm{X}}$, and $N_{\mathrm{H}}$ and $L_{\mathrm{X}}$, this is a strong indication that the X-ray variability observed during the outburst of V1118 Ori is only caused by the amount of material in the corona. It is, thus, probably related to the amount of mass falling from the disk into the stellar magnetosphere, i.e., to the mass accretion rate.

\section{Optical and infrared analysis}

\subsection{Silicate feature}

Figure 9 shows the silicate feature obtained at the three different epochs and its normalized version $\left(S_{v}=1+F_{v} / F_{\mathrm{C}}\right)$. We have used a linear fit to the SL spectra in the $\log \lambda-\log F_{v}$ plane, using only wavelength ranges of $6.0-8.0 \mu \mathrm{m}$ and $13.0-13.5 \mu \mathrm{m}$ to determine the underlying continuum flux level, $F_{\mathrm{C}}$. The shapes of the silicate feature in the three observations are compatible with one another, but the Mar. 2005 silicate feature appears to be brighter than the Feb. 2005 or post-outburst (Nov. 2008) normalized fluxes, i.e., more flux is coming from the less optically thick disk upper layers, likely as a result of hotter temperature of such layers. The heating of the upper layers is probably caused by the strong irradiation, e.g., by a hot spot (see below). The ratio $S_{11.3} / S_{9.8}$ is $0.95-1.0$, suggesting grain growth, and the ratio $\left(S_{11.3} / S_{9.8}\right) /\left(S_{\text {peak }}^{10 \mu \mathrm{m}}\right)$ is $0.74-0.79$, evidence of a flat silicate feature.

In the outburst spectrum of the EXor prototype EX Lup, Ábrahám et al. (2009) observed a silicate feature with a crystalline peak, in contrast to the pre-outburst silicate feature which was similar to the interstellar medium spectrum dominated by amorphous grains. They concluded that thermal annealing in the surface layer of the proto-planetary disk was the mechanism for this change in spectral features. Unfortunately, we have no pre-outburst spectrum of V1118 Ori to compare with the outburst spectra, only a spectrum taken 2 years after the end of the outburst and 3.5 years after our last outburst spectrum in March 2005. If the pre-outburst spectrum was indeed typical of amorphous silicates, it means that the time scale for disappearance of the crystalline features must be longer than 2 years. Ábrahám et al. (2009) proposed that similar crystallization must have occurred during the previous 1955-1956 outburst of EX Lup, and since the 2005 quiescent spectrum did not show crystalline features, the removal time scale must have been less than 50 years. On the other hand, in V1118 Ori, it is also possible that the unchanging silicate feature in the three spectra is typical of the quiescent silicate feature in the young star, perhaps 

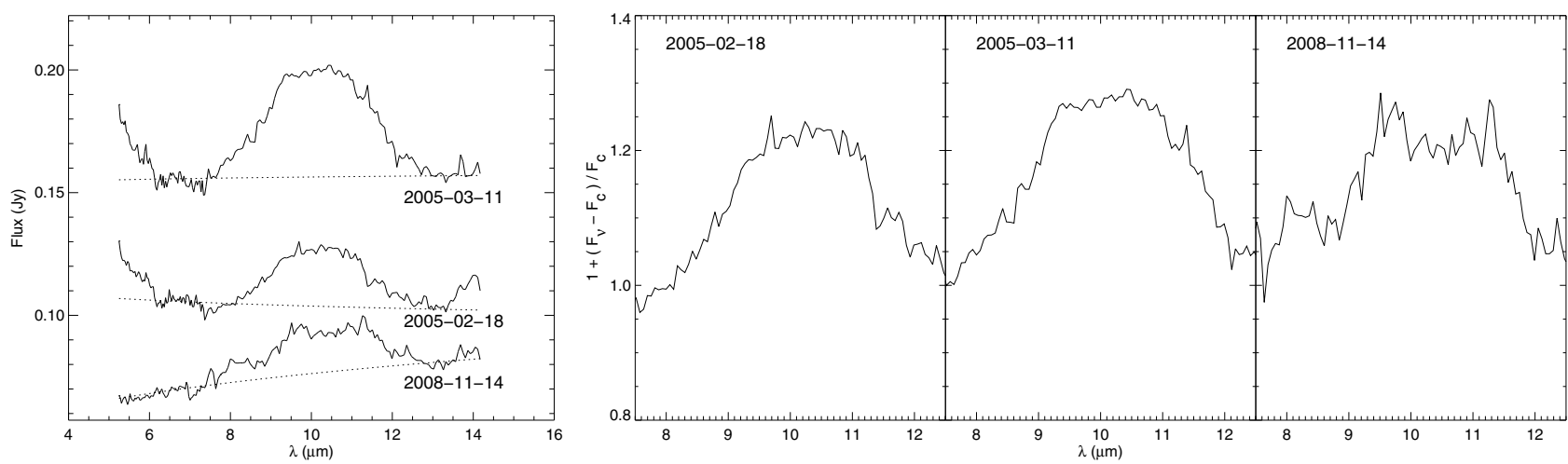

Fig. 9. Spitzer IRS SL spectra of V1118 Ori for the three epochs (left panel). The dotted line shows the fit to the underlying continuum in the $[6-8,13-14.5] \mu \mathrm{m}$ range. The right panels show the normalized flux at the three epochs.
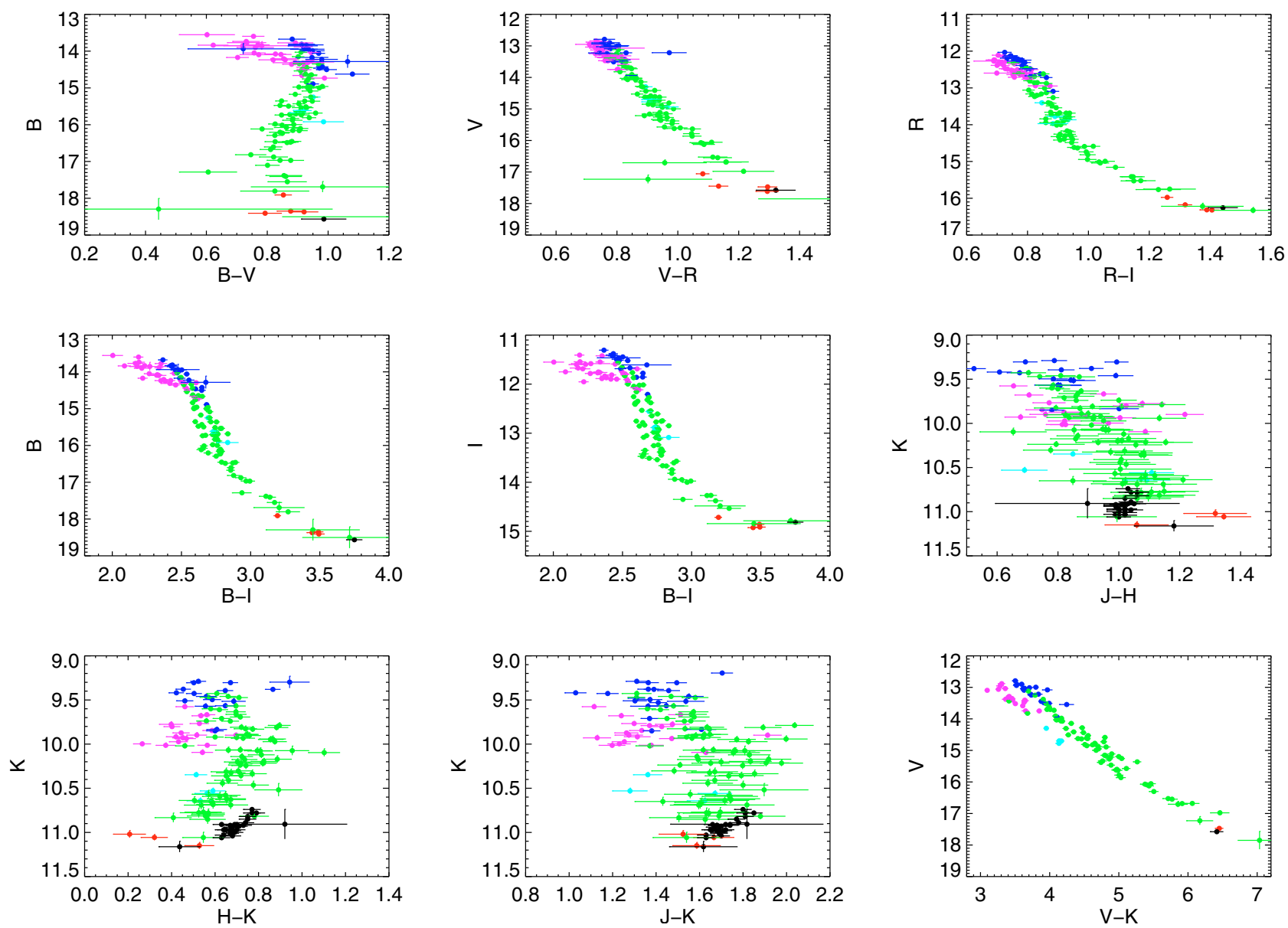

Fig. 10. Magnitude versus color indices for the different time intervals (red: \#1, lime: \#2, violet: \#3, blue: \#4, green: \#5, black: \#6).

an indication that the disk is more evolved. In any case, M-type young stars typically show flat silicate features (e.g., KesslerSilacci et al. 2005, 2006). Finally, the IRS data do not show strong evidence of the forsterite crystalline feature at $16 \mu \mathrm{m}$, in contrast to the feature observed in EX Lup (Ábrahám et al. 2009). This is consistent with the absences of a sharp peak at $10 \mu \mathrm{m}$ and of a shoulder at $11.3 \mu \mathrm{m}$.

\subsection{Color indices}

We have calculated color indices from our optical and nearinfrared data, and looked into their evolution during the outburst.
Time intervals were defined in the pre-outburst epoch $(1 ; \mathrm{JD}<$ $2453200)$, initial phase of rise $(2 ; 2453200<$ JD $<2453390)$, second phase of rise $(3 ; 2453390<$ JD $<2453450)$, plateau (4; $2453450<\mathrm{JD}<2453650)$, decay $(5 ; 2453650<\mathrm{JD}<$ $2453900)$, and post-outburst $(6 ; 2453900<\mathrm{JD}<2454200)$.

Figure 10 shows the color-magnitude diagrams, whereas Fig. 11 shows the color-color diagrams, together with an $A_{V}=$ 1 mag reddening vector using the reddening law of Rieke et al. (1985), the loci of the unreddened main sequence and giant stars (Bessell \& Brett 1988), and the loci of classical T Tauri stars (Meyer et al. 1997). Compared to the pre-outburst color indices of V1118 Ori, the optical color indices are bluer in the early 

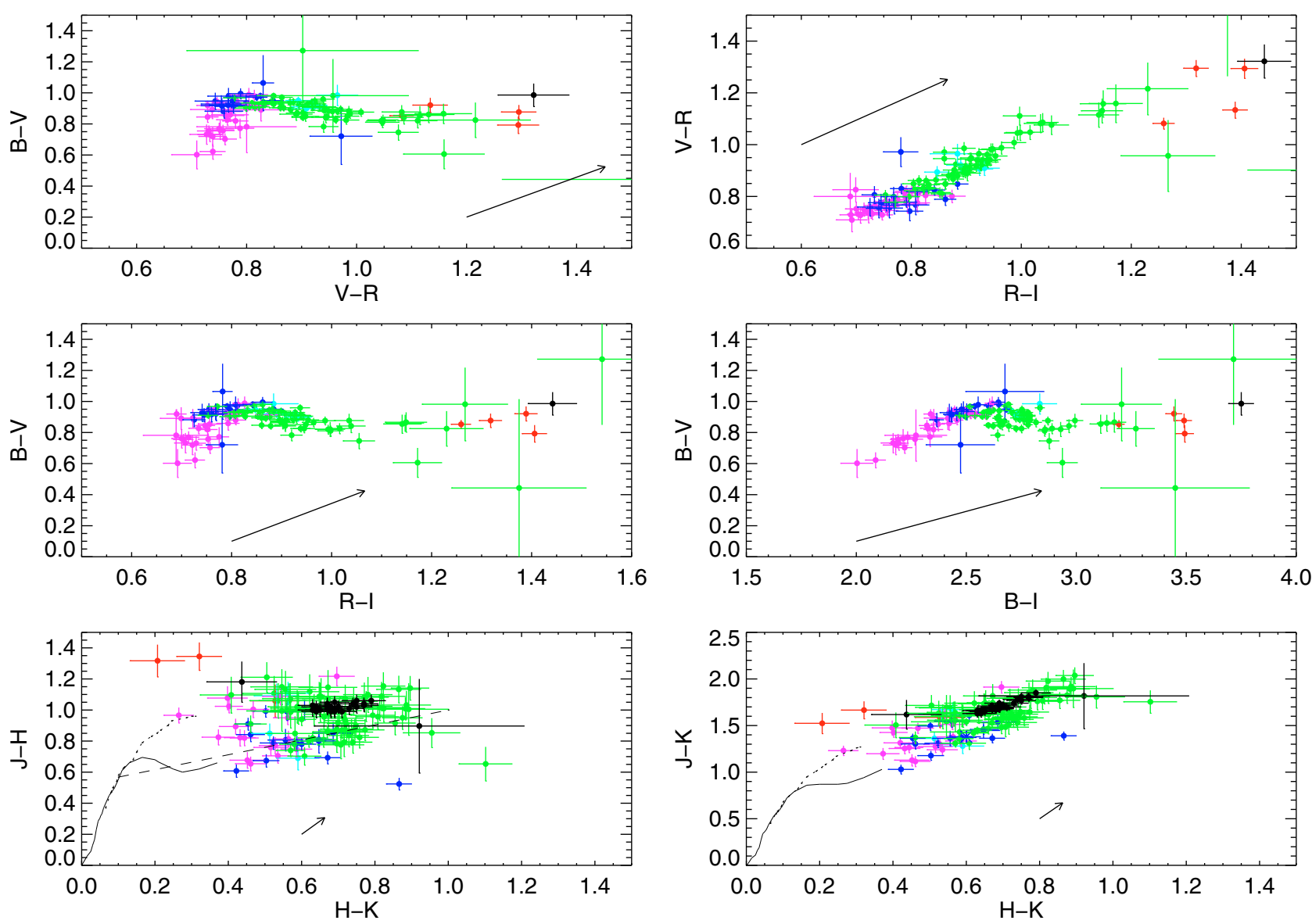

Fig. 11. Color index diagrams for the same time intervals as in Fig. 10. An $A_{V}=1$ mag vector is added using the reddening law of Rieke et al. (1985). The loci of unreddened main sequence and giant stars (Bessell \& Brett 1988) are shown for the near-infrared panels as solid and dotted curves, respectively. Finally the loci of classical T Tauri stars (Meyer et al. 1997) are shown as a dashed line in the $(J-H)$ vs. $(H-K)$ diagram.

phases of the outburst (except for $B-V$ which shows a peculiar pattern, probably because the $B$ magnitude is very sensitive to the evolution of hotspot emission), and gradually return to the pre-outburst values in the decaying phase of the outburst. In the near infrared, an apparent blueing occurs as well in the initial phases of the outburst with a return to "normal" colors in the decay phase. The above results are consistent with the hypothesis of Lorenzetti et al. (2007) that blueing must have occurred because of a hotter temperature component during the initial phase of the outburst (their data were taken only in the decaying phase and showed reddening). The variations in the color-color diagrams also show no trend related to extinction variations, in agreement with the suggestion of Lorenzetti et al. (2007) and Lorenzetti et al. (2009). There is, however, a larger scatter in the infrared color-color diagrams, especially in $J-H$ versus $H-K$. Since the stellar photospheric emission does not vary during the outburst, we need to understand the variations in color indices and magnitudes during the outburst in the context of a star + disk + hot spot model. Lorenzetti et al. (2007) suggested that the polarization found in the $I$ band of the V1118 Ori outburst was intrinsic and that it was probably caused by the spotted and magnetized stellar photosphere. As we will show below, the photometric changes can indeed be explained by changes in the fluxes of the disk thermal emission and from a hot spot as the rate of mass falling from the disk onto the stellar photosphere increases during the outburst. We note also that during the outburst, any increase in hotspot emission will further increase the irradiation of the disk. The following section aims to understand the multi-wavelength photometric and spectroscopic behavior observed during the outburst of V1118 Ori by comparing the data with disk models of young stars.

\subsection{Spectral energy distribution}

Figure 12 shows the observed SED for V1118 Ori. We have shown as small colored dots the data for the different phases (as defined at the beginning of Sect. 5.2 and in the caption of Fig. 10), and the pre- and post-outburst photometry as red crosses and black stars, respectively. The IRS low-resolution spectra are also shown for the outburst phases (violet) and postoutburst phase (black). For clarity, we do not show the highresolution spectra. To emphasize the expected stellar photospheric contribution, we have added a stellar atmosphere model from the PHOENIX database ${ }^{4}$ (e.g., Hauschildt et al. 1999) for a star with $T_{\text {eff }}=3600 \mathrm{~K}, \log g=4.0$, and $Z=0$ (STARdusty 2000 models), and reddened it with $A_{V}=1.5 \mathrm{mag}$ and the reddening law of Fitzpatrick (1999). Indeed, the hydrogen column density derived from X-rays is $2.7_{-0.9}^{+1.2} \times 10^{21} \mathrm{~cm}^{-2}$ in 2002 , and does not change much during the outburst, generally staying in the range $1-5 \times 10^{21} \mathrm{~cm}^{-2}$, which corresponds to $A_{V}$ in the range $A_{V}=1.71_{-0.57}^{+0.76} \mathrm{mag}$ for $R_{V}=3.1$ (Galactic value) or $A_{V}=1.44_{-0.48}^{+0.64}$ mag for $R_{V}=5.5$ (dusty environment).

\footnotetext{
4 http://www.hs.uni-hamburg.de/EN/For/ThA/phoenix/ index.html
} 


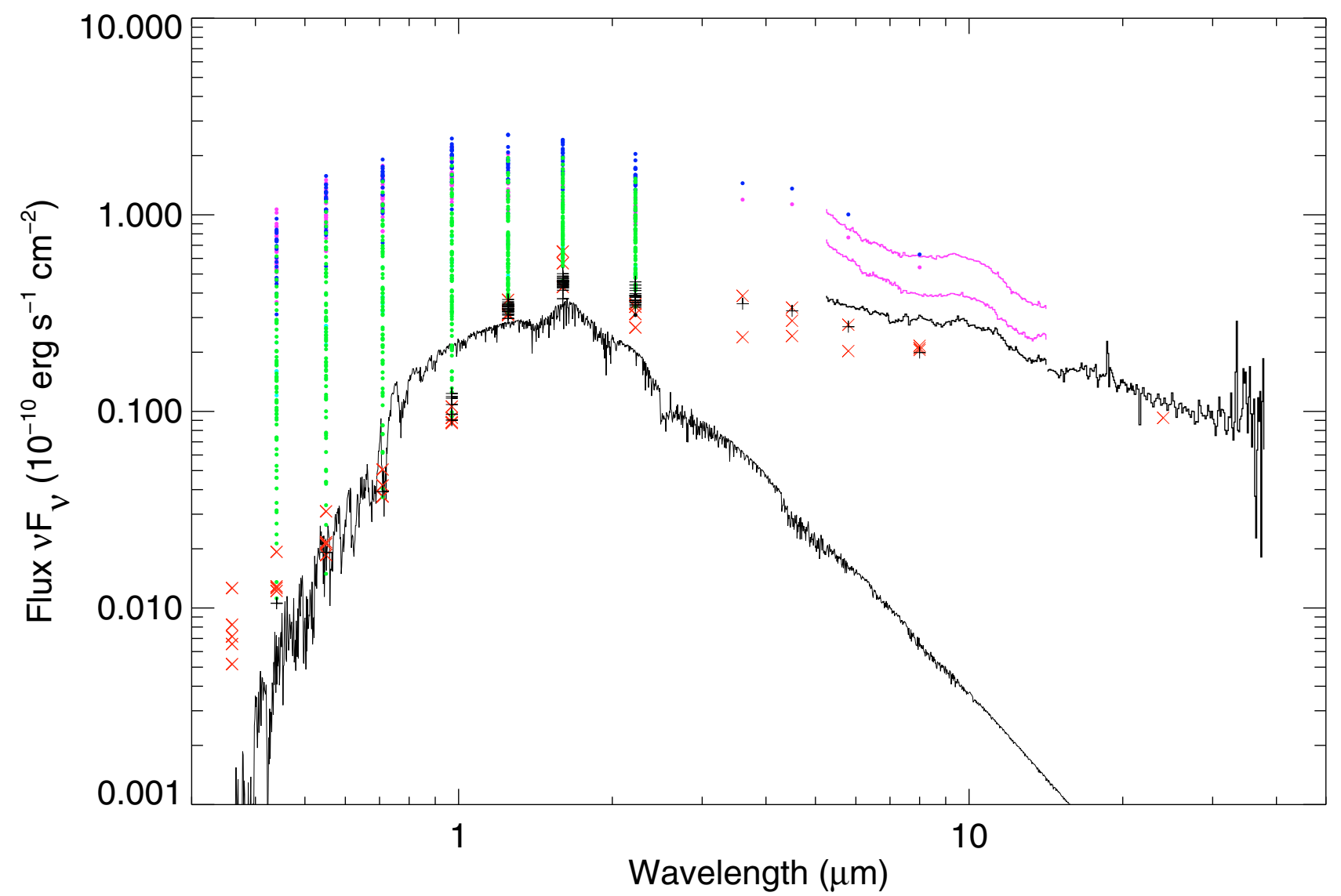

Fig. 12. Compilation of the optical and infrared photometry taken before, during, and just after the outburst (see text and caption of Fig. 10 for details about colors). The IRS SL and LL spectra and a reddened $\left(A_{V}=1.5 \mathrm{mag}\right)$ stellar atmosphere model are also shown.

We calculated the median magnitude differences from 0.44 to $8.0 \mu \mathrm{m}$ (we have no $U$ photometry during the outburst), relative to the median magnitude in quiescence (we used both preoutburst and post-outburst magnitude; Fig. 13). There is clear evidence that the optical emission brightened much more than the infrared emission, with a peak in the $V$ band. We also observe that the $B V R I$ bands decayed faster than the $J H K$ bands, probably indicating a mixture of different emission components in the near-infrared bands (e.g., disk and hot spot). At long wavelengths $(\lambda \geq 2 \mu \mathrm{m})$, the increase in flux was similar at all wavelengths.

To quantify better the star+disk properties of V1118 Ori before and during its outburst, we have then used the code described in Whitney et al. (2003a,b), version $20090224^{5}$. We have run the code by fixing certain parameters and leaving others free to vary. The models did not include an envelope, but only an ambient medium with density of $10^{-22} \mathrm{~g} \mathrm{~cm}^{-3}$. The contribution of this medium is generally negligible. The exclusion of the envelope is motivated by its contributing mostly at long wavelengths where we have no data. The IRS data also do not show strong evidence of an envelope (that could not be attributed to unreliable background subtraction).

The fixed parameters were the stellar radius $\left(R_{\star}=1.29 R_{\odot}\right)$, mass $\left(M_{\star}=0.41 M_{\odot}\right)$, and effective temperature $\left(T_{\mathrm{eff}}=3600 \mathrm{~K}\right.$; see Sect. 2; we used as input a lower spectral resolution version of the same PHOENIX stellar atmosphere model as above), the disk mass $\left(5 M_{\text {Jup }}\right)$ and outer radius (100 AU), the disk flaring power $\left(\propto R^{\beta}, \beta=1.1\right)$, the disk scale height $\left(0.01 R_{\star}\right.$ at $R_{\star}$; the

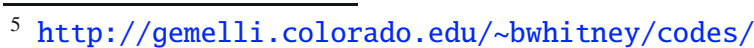

height is thus $2.6 \mathrm{AU}$ at $100 \mathrm{AU}$ ), and the disk density profile $\left(\propto R^{-\alpha}, \alpha=2.1\right)$. For the dust opacity, we used a model of grains as large as $1 \mathrm{~mm}$ for the denser regions of the disk (see Wood et al. 2002 for $\mathrm{HH}$ 30), but used an interstellar medium grain model for the less dense regions of the disk (i.e., below a threshold set at $3.34 \times 10^{-14} \mathrm{~g} \mathrm{~cm}^{-3}$ ). The disk mass and outer radius were not constrained by our optical and infrared data ${ }^{6}$. Changes in their values would have little impact on the models at opticalinfrared wavelengths.

We have left the mass accretion rate and inner disk radius (in units of the dust destruction radius, $R_{\mathrm{sub}}$ ) free to vary. The model also includes the possibility for material to fall from the disk onto a hot spot by following the magnetic field lines. We have kept the truncation radius to $5 \mathrm{AU}$, but left the fractional area, $f_{\mathrm{S}}$ (ratio of the shock area to the star area; see Calvet \& Gullbring 1998), free to vary. Finally, the code samples the model at 10 different inclination angles from $\cos i=0.05$ to 0.95 every 0.10 , i.e., from $87^{\circ}$ to $18^{\circ}$. Thus, we have four different parameters that can vary $\left(\left[\cos i, \dot{M}, f_{\mathrm{S}}, R_{\min }\right]\right)$.

The choice of $\alpha$ and $\beta$ is typical of those observed in T Tauri stars, and correspond to values that seem to fit the V1118 Ori data. The power law indices are related in the sense that if the

\footnotetext{
6 We obtained data with the IRAM $30 \mathrm{~m}$ MAMBO2 bolometer at $1.2 \mathrm{~mm}$ in late November 2009. V1118 Ori was detected at $1.8 \pm 0.4 \mathrm{mJy}$. Assuming an optically thin regime, an opacity of $\kappa_{v}=0.02 \mathrm{~cm}^{2} \mathrm{~g}^{-1}$ and using a gas-to-dust ratio of 100 , we obtained a disk mass of $2.45 \pm 0.5 M_{\text {Jup }}$ for a characteristic temperature of $20 \mathrm{~K}$. The disk mass increased to $6.9 \pm 1.5 M_{\text {Jup }}$ for $T_{\mathrm{c}}=10 \mathrm{~K}$. Although the determination of the exact disk mass is out of scope in this paper, the derived estimates indicate that the assumed disk mass in our model is realistic.
} 


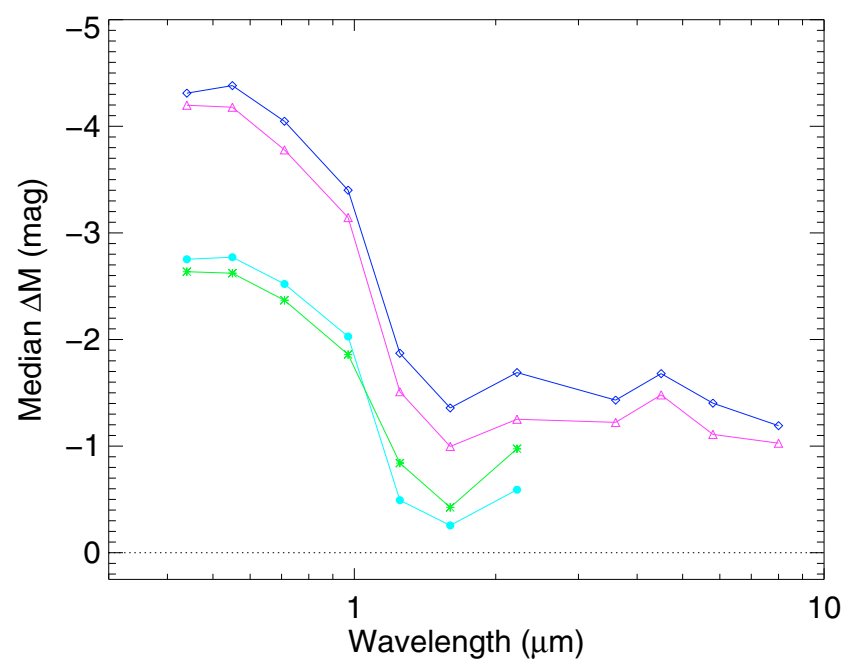

Fig. 13. Median magnitude difference relative to quiescence as a function of wavelength. The same time intervals as in Fig. 10 are used for the color coding (lime filled circles: \#2, violet triangles: \#3, blue diamonds: \#4, green stars: \#5). In the initial phase of the outburst, a strong optical component developed, with a peak in the $V$ band.

disk surface density is $\Sigma \propto R^{p}$, then $p=\beta-\alpha$. A value of $\beta=$ 1.1 is close to the theoretical values for irradiated and $\alpha$ disks $(\beta=9 / 8=1.125)$, and, with $\alpha=2.1, p=\beta-\alpha=-1.0$, i.e., typical for a steady optically thick disk. A fit to the IRAC and MIPS fluxes in quiescence gives $\lambda F_{\lambda} \propto \lambda^{-0.65}$, i.e., consistent with the commonly observed $\lambda F_{\lambda} \propto \lambda^{-2 / 3}$ for irradiated disks. Assuming that the temperature follows a power law $T \propto R^{-q}$, and if $\lambda F_{\lambda} \propto \lambda^{s}$, both indices are related by $s=2 / q-4$. Thus, we have $q=0.6$, i.e., $T \propto R^{-3 / 5}$.

There is no pre-outburst IRS spectrum available to determine the shape of the silicate feature before the outburst (emission or absorption). However, we have used the post-outburst IRS spectra, scaled by a factor of 0.7 to match the IRAC photometry (see Fig. 9). For the photometry, we have used the median of the preoutburst (interval \#1) and post-outburst (interval \#6) photometry. For the outburst data, we have used the plateau (\#4) peak photometric data with the second epoch outburst IRS SL spectrum to characterize the SED at "peak". The IRS spectrum already matches well the $8 \mu \mathrm{m}$ flux without correction and shows the silicate feature in emission.

We ran the code with 5000000 "photons" or energy packets for each model. This results in SED models with reasonably good signal-to-noise ratios over the wavelength range covered by our data. The models were then reddened with $A_{V}=1.5 \mathrm{mag}$ (assuming ISM opacities). For each realization we have calculated a reduced $\chi_{p}^{2}$ value

$\chi_{p}^{2}=\frac{1}{N} \sum_{i=1}^{N}\left(\frac{\log _{10} F_{v}\left(\lambda_{i}\right)-\log _{10} M_{v}\left(\lambda_{i}, \boldsymbol{p}\right)}{\Delta\left(\log _{10} F_{v}\left(\lambda_{i}\right)\right)}\right)^{2}$

where $F_{v}\left(\lambda_{i}\right)$ are the data flux densities, $M_{v}\left(\lambda_{i}, \boldsymbol{p}\right)$ are the model flux densities (for parameter vector $\boldsymbol{p}=\left[\cos i, \dot{M}, f_{\mathrm{S}}, R_{\min }\right]$ ) interpolated at the wavelength of the data points $(0.44,0.55,0.71,0.97,1.25,1.60,2.22,3.6,4.5,5.8,8.0,24.0 \mu \mathrm{m}$ for the photometry, and $5.5,6.0,6.5, \ldots, 14.0 \mu \mathrm{m}$ for the IRS - we only used the SL data). For the photometric flux density uncertainties, we have used a fixed $\Delta\left(\log _{10} F_{v}\left(\lambda_{i}\right)\right)=0.05$, i.e.,
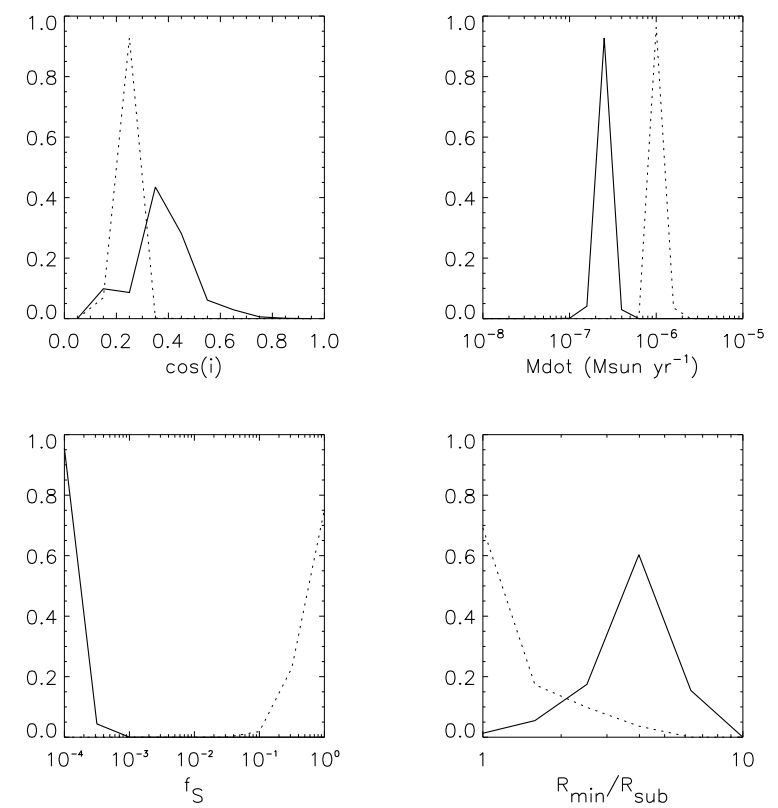

Fig. 14. Probability distribution of the parameters ([cos $\left.\left.i, \dot{M}, f_{\mathrm{S}}, R_{\min }\right]\right)$ for the quiescent (solid) and outburst (dotted) cases.

about $10 \%$ relative uncertainties for all data points (a value larger than many actual uncertainties on the measured values) to account for flux variations observed before and at the peak of the outburst. We used $\Delta\left(\log _{10} F_{v}\left(\lambda_{i}\right)\right)=0.025$ instead for the IRS SL data to put more weight on the silicate feature.

Figure 14 shows the normalized model probabilities (calculated as $p_{p}=\mathrm{e}^{-\chi_{p}^{2}}$ ) for the quiescent case (solid), and for the outburst case (dotted). The distributions of inclination angles are slightly different for the quiescent and peak of outburst cases, but the distributions overlap in the range $\cos i=0.1-0.3$, with a maximum probability (obtained by multiplying the probability distributions) at $\cos i=0.25$, i.e., $i \approx 76^{\circ}$. The mass accretion rate distributions are sharply peaked: the most probable values of $\dot{M}$ are $10^{-6.6} M_{\odot} \mathrm{yr}^{-1}$ and $10^{-6.0} M_{\odot} \mathrm{yr}^{-1}$ in quiescence and and at the peak of the outburst, respectively, indicating an increase by a factor of 4 . The coverage factor seems to be small $\left(f_{\mathrm{S}}<0.0001\right)$ outside the outburst while the simulations indicate high values $\left(0.1<f_{\mathrm{S}}<1\right)$ at the peak of the outburst. This parameter is essentially constrained by the optical and nearinfrared photometry. The minimum disk radius has likely values of $R_{\min }=\left(10^{0.4}-10^{0.8}\right) R_{\text {sub }}$, with the most probable value at $R_{\text {min }} \approx 4 R_{\text {sub }}$, whereas the values at the peak of the outburst are lower and most likely equal to the sublimation radius. The best models give sublimation radii of about 0.10 AU in quiescence and $0.20 \mathrm{AU}$ at the peak of the outburst, implying inner disk radii of $0.25-0.6 \mathrm{AU}$ (most probable value of $0.4 \mathrm{AU}$ ) and $0.2 \mathrm{AU}$, respectively. This result suggests that the inner disk radius (determined from the infrared data arising from dust emission) probably diminished during the outburst, although we emphasize that quiescent values of $0.2 \mathrm{AU}$ are possible, though less probable from our SED modeling.

Figure 15 shows the best fits to the "quiescent" SED and to the peak of outburst SED, respectively. The total (bolometric) luminosity (including all components) varied from about $2.0 \mathrm{~L}_{\odot}$ in quiescence (with a contribution of the stellar luminosity, $L=4 \pi R^{2} \sigma T_{\mathrm{eff}}^{4}$, of $0.25 L_{\odot}$ and of the thermal disk luminosity 

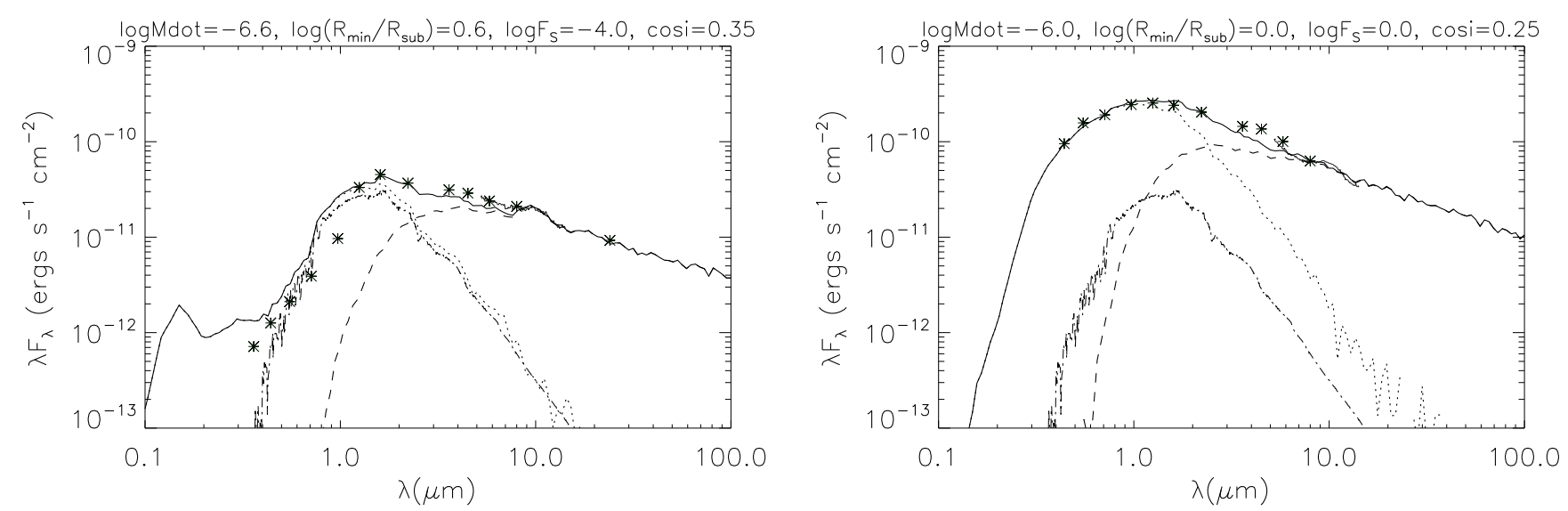

Fig. 15. The best-fit models to the "quiescent" SED (left) and to the peak outburst SED (right). The direct stellar contribution (including emission by the hot spot) is shown as a dotted line (the $A_{V}=1.5$ mag reddened stellar atmosphere model is shown as a dash-dotted line), the thermal disk spectrum as a dashed line.

of $0.9 L_{\odot}$ ) to $\approx 7.4 L_{\odot}$ at the peak of the outburst ${ }^{7}$ (the thermal disk luminosity increasing to about $1.7 L_{\odot}$ ).

Thus the bolometric luminosity is dominated by the stellar direct emission, i.e., the stellar photospheric emission and the hotspot emission (the latter in particular during the outburst). We note, however, that we would need data at shorter wavelengths than $B$ to constrain better the hotspot emission during the outburst.

\section{Discussion}

Our multi-wavelength campaign has allowed us to obtain several pieces of information on the outburst of V1118 Ori, as seen in the optical and infrared, and in the X-rays. Overall, the evolution of the X-ray flux during the outburst followed the evolution of the optical and near-infrared fluxes. The sampling of the X-ray observation does not allow us to determine whether the increase in X-ray flux in March 2006 is related to the shortterm increase in optical/infrared flux observed about 45 days earlier. The thermal properties of V1118 Ori showed evidence of a plasma with temperature of a few MK to a few tens of MK. In our initial paper, we reported a change in the spectral properties from a predominantly hot $(20 \mathrm{MK})$ plasma before the outburst (and possibly at the very beginning) to a lower temperature (8 MK) in February 2005 (Audard et al. 2005b). Our reanalysis confirms this finding and a gradual return to higher values in the later phases of the outburst. However, the low signal-to-noise ratio of the X-ray spectra taken during our monitoring campaign makes it difficult to accurately constrain the plasma temperature (in contrast, the February and March 2005 observations had a much better signal-to-noise ratio). The alternative quantile analysis showed evidence of less variation in the plasma temperature. Nevertheless, it is consistent with the detected change in temperature. While V1647 Ori showed a strong increase in X-ray flux during the outburst (Kastner et al. 2004; Grosso et al. 2005),

\footnotetext{
7 Lorenzetti et al. (2006) quote a peak luminosity of $25.4 L_{\odot}$; however, they used the peak magnitudes of a previous outburst to build the outburst peak SED and to derive the luminosity. The previous outburst had a peak magnitude of $J=8.5$, in contrast to the 2005-2006 outburst that peaked at $J=10.6$. In addition, our method derives the luminosity by matching the observed SED with a detailed radiative transfer model, while Lorenzetti et al. (2006) integrated the flux densities. The two issues may explain the difference in derived luminosities.
}

V1118 Ori showed only a moderate X-ray flux increase, and possibly an eventual decrease in flux after the outburst, if we assume that the 2002 flux level was representative of the preoutburst X-ray flux. In this case, the impact of the mass infall onto the stellar magnetosphere must have significantly affected the latter, leaving it in a lower X-ray flux state. It is, however, also possible that the 2002 flux was observed by chance in a high quiescent state, and that the $2007 \mathrm{X}$-ray flux is closer to the pre-outburst flux level. In any case, the changes in the X-ray fluxes during the outburst, and possibly the temperature changes all indicate a strong interplay between the material falling from the disk as a consequence of the increase in mass accretion rate and the stellar magnetosphere.

From the optical and infrared SED data and Monte-Carlo models using the code of Whitney et al. (2003a,b), there is clear evidence that the thermal disk emission dominates in the midinfrared in quiescence and during the outburst, while emission from a hotspot and reprocessed emission dominates the outburst SED in the optical and near-infrared, and contributes to some extent before the outburst. However, this conclusion depends on our choice of using disk models that stop at the sublimation radius, i.e., there is no disk contribution at smaller radii, where the gas is optically thin. This could contribute to the near-infrared fluxes, but it should not contribute significantly to the optical fluxes. Therefore, we believe that the addition of hotspot emission is necessary to fit the blue side of the V1118 Ori SED, during the outburst and probably before the outburst.

The increase in mass accretion rate during the outburst leads to the change in inner disk radius which moves closer to the star's surface. Such an effect could explain the changes in the $\mathrm{X}$-ray emission (see above). From our simulations, we have determined that the mass accretion rate increased during the outburst: consequently, the dust sublimation radius increased from $\approx 17 R_{\star}=0.1 \mathrm{AU}$ to $\approx 32 R_{\star}=0.2 \mathrm{AU}$, whereas the inner disk radius (based on the infrared dust emission) moved from $0.4 \mathrm{AU}$ in quiescence to $0.2 \mathrm{AU}$ in outburst (although the optically thin gas disk could be closer to the star), and the inner disk radius is consistent with the dust sublimation radius at the peak of the outburst. We emphasize that such estimates of the inner disk radius are biased by our use of dust thermal emission for the disk. The optically thin gas disk is difficult to detect and we would require gas tracers, e.g., $\mathrm{H}_{2}$ or $\mathrm{CO}$ bandheads to properly determine the inner extent of the disk. In any case, the strong increase in fractional area of the hotspot suggests that large amounts of matter 
fell from the disk onto the star during the outburst, incidentally interacting with the stellar magnetosphere.

Using their near-infrared spectrum taken in September 2005 (i.e., about at the end of the plateau phase) and a wind loss model, Lorenzetti et al. (2006) derived a mass loss rate ${ }^{8}$ of about $4 \times 10^{-8} M_{\odot} \mathrm{yr}^{-1}$ from H I. Furthermore, little or no absorption was reported $\left(A_{V}<2\right)$, with no evidence of variations during the outburst. They also derived a mass loss rate of (3-8) $\times 10^{-7} M_{\odot} \mathrm{yr}^{-1}$ from the CO overtone emission, indicating an ionization fraction of $0.1-0.2$. Furthermore, Lorenzetti et al. (2007) observed V1118 Ori in September 2006, i.e., well after the outburst, and detected no emission line, while the $2.3 \mu \mathrm{m}$ CO band was seen in absorption. Herbig (2008) reported evidence of wind emission in $\mathrm{H} \alpha$ ( $\mathrm{P}$ Cyg profile) in November 2005, in contrast with the more symmetric profile observed after the outburst in December 2006. It is, therefore, likely that high wind loss rates occurred transiently because of the instability in the disk. The disk emission may provide a better fit to $\mathrm{CO}$ bands in young stars than mass loss emission in general (e.g., Chandler et al. 1995), but this may not be the case during the outburst of a young star such as V1118 Ori: our SED models including emission due to matter falling from the accretion disk onto the stellar photosphere clearly show that the outburst SED at wavelengths below $3 \mu \mathrm{m}$ is dominated by the hotspot emission, not by disk thermal emission (while it remains negligible outside the outburst and stellar photospheric emission dominates). Wind loss emission may thus be present in the optical and near-infrared.

We can compare the mass loss rates derived for the neutral gas by Lorenzetti et al. (2006) to our derived mass accretion rates: the SED modeling suggests a variation in the mass accretion rate from about $2.5 \times 10^{-7} M_{\odot} \mathrm{yr}^{-1}$ to $1.0 \times 10^{-6} M_{\odot} \mathrm{yr}^{-1}$, i.e., these mass accretion rates are comparable to the mass loss rates determined by Lorenzetti et al. (2006). This is somewhat surprising, since mass outflow rates are expected to be about ten times lower than mass accretion rates (Hartigan et al. 1995, with revised mass accretion rates, see Gullbring et al. 1998; Edwards 2009). Our mass accretion rate in quiescence is larger by about a factor of 10 compared to those derived for classical T Tau stars $\left(\approx 10^{-8} M_{\odot} \mathrm{yr}^{-1}\right.$; e.g., Gullbring et al. 1998), and lower by a factor of 10 than the rates derived for embedded Class I stars $\left(\approx 5 \times 10^{-6} M_{\odot} \mathrm{yr}^{-1}\right.$; Kenyon et al. 1993). Therefore, our derived mass accretion rates are consistent with placing V1118 Ori in a category between Class I and CTTS stars. We note also that the IRAC color indices of V1118 Ori in quiescence are $[3.6]-[4.5]=0.65-0.75$ and $[5.8]-[8.0]=0.65-0.7$ which places it formally in the region of Class II sources, but also close to the region for Class 0/I (e.g., Allen et al. 2004). Furthermore, the $K-[3.6]$ color of 1.52 in "quiescence" also place the star as a strong accretor. In contrast, $U-V=-0.05$ which formally would (incorrectly) place V1118 Ori as a weak-lined T Tauri star. In fact, using the Gullbring et al. (1998) method to derive mass accretion rates from the $U$-band luminosity excess, we find a mass accretion rate of $4.1 \times 10^{-9} M_{\odot} \mathrm{yr}^{-1}$, much smaller than values derived by SED modeling, and also inconsistent with the strong infrared excess. While our derived mass accretion rates are model-dependent, we are confident that our derived mass accretion rates are realistic, despite the difficulty to derive mass

\footnotetext{
8 Lorenzetti et al. (2006) derive the mass loss rate from the wind model of Carr (1989) and his Fig. 8. While their derived mass loss rate is accurate, they incorrectly give a CO luminosity of $6.3 \times 10^{-5} L_{\odot}$, while it should have been $7.9 \times 10^{-4} L_{\odot}$ based on the quoted line flux (Lorenzetti 2009, priv. comm.).
}

accretion rates from infrared excess (e.g., Kenyon \& Hartmann 1987).

Overall, the data provide ample evidence that a significant portion of matter fell from the disk onto the young star as a consequence of the increase in mass accretion rate. Over a duration of about 400 days, we estimate about $5 \times 10^{-7} M_{\odot}=0.18 M_{\oplus}$ was deposited (using an average of half the peak mass accretion rate), i.e., about twice the amount that would have been deposited over the same time range if using the quiescent mass accretion rate The hotspot covering factor we derive at the peak of the outburst is also relatively large $\left(f_{\mathrm{S}}>0.1\right)$, suggesting that a large fraction of the stellar magnetosphere may also have been influenced by the increase in mass accretion rate, which in turn supports the hypothesis that the stellar magnetosphere was modified during the outburst and the X-ray properties of the coronal plasma have changed Audard et al. (2005b). Our SED modeling of the dust thermal emission suggests a change of the inner dust disk radius from about $0.4 \mathrm{AU}$ in quiescence to $0.2 \mathrm{AU}$ at the peak of the outburst (the optically thin gas could reach down to $0.2 \mathrm{AU}$ or below before the outburst, although it would be unclear why dust would not go down to the sublimation radius, unless, e.g., disk clearing by a giant planet or significant grain growth making the dust optically thin would be present in the inner portion of the disk). Together with the increase in $f_{\mathrm{S}}$, this provides evidence that the disk closed in during the outburst, disrupted the magnetosphere, and matter fell onto the stellar photosphere, producing strong hotspot emission that dominated the optical and near-infrared in outburst (while the thermal disk emission increased by a factor of 4 and dominated the mid-infrared emission).

While color-color and color-magnitude diagrams can be used to determine the level of reddening in young stars, such diagrams might be difficult to use to measure any reddening during the outburst of a young star: in addition to reddening, the whole SED of a young star changes dramatically, increasing in flux, but also in shape: in the case of V1118 Ori, the optical and near-infrared fluxes increased much more than the mid-infrared spectrum. The former are dominated by stellar and hotspot emission, while the latter is dominated by thermal disk emission. Color-color diagrams show a clear blueing of the colors (Fig. 11), not caused by clearing of any enshrouding material, but by the strong increase in flux below $1.5 \mu \mathrm{m}$ (Fig. 13). Such multi-wavelength data show that a good coverage of the SED is necessary to understand the disk-star interactions in young stars. Furthermore, Figures 10 and 11 indicate that V1118 Ori returned (within uncertainties) to its "initial", pre-outburst conditions.

\section{Conclusions}

We have followed the young accreting star V1118 Ori before, during, and after its outburst in 2005-2006 with photometry in UBVRIJHK and in X-rays with XMM-Newton and Chandra. We have further obtained Spitzer photometry and spectroscopy during the outburst and after the outburst.

The optical and infrared data showed significant variations, with brightening by as much as about 3-4 mag below $1 \mu \mathrm{m}$, and up to $\approx 2 \mathrm{mag}$ in the infrared. Monte-Carlo simulations of a star+disk+hotspot model suggested that the optical data were dominated by hotspot emission at the peak of the outburst (while the stellar photosphere dominated before the outburst), while thermal disk emission from optically thick dust dominated the infrared. The SED analysis showed an increase in mass accretion rate from $\dot{M}=2.5 \times 10^{-7} M_{\odot} \mathrm{yr}^{-1}$ in quiescence to $\dot{M}=1.0 \times 10^{-6} M_{\odot} \mathrm{yr}^{-1}$ at the peak of the outburst, and an increase of the hotspot fractional coverage area from $f_{\mathrm{S}} \leq 10^{-4}$ 
to $f_{\mathrm{S}}>0.1$. The SED modeling suggests that the dust sublimation radius increased from 0.1 to $0.2 \mathrm{AU}$. Based on the thermal dust emission, the inner disk radius moved from about 4 times the dust sublimation radius $(0.4 \mathrm{AU})$ to $0.2 \mathrm{AU}\left(=R_{\mathrm{sub}}\right)$ at the peak of the outburst, although the gas inner disk radius could extend within the dust sublimation radius. The inclination angle of the disk is constrained to values of $\cos i=0.1-0.3$ with a peak at 0.25 , i.e., $i \approx 76^{\circ}$.

The Spitzer IRS spectra showed the silicate feature at $10 \mu \mathrm{m}$ in emission during and after the outburst, with no apparent variation in shape or flux, indicating that the outburst had little impact on the optically thin region where the silicate emission originates. Emission lines were detected in the high-resolution module data although the background subtraction proved to be difficult, especially for the outburst spectra. Follow-up observations, e.g., from the ground should confirm the detection of lines of particular interest, e.g., [Ne II], [S III], and $\mathrm{H}_{2} 0-0$.

The initial X-ray observations (Jan.-Mar. 05) indicated a change in the thermal structure of the coronal spectrum of V1118 Ori with little change in the X-ray flux (Audard et al. 2005b), while fading in the X-rays was already detected in September 2005 (Lorenzetti et al. 2006). Our continued monitoring with XMM-Newton and Chandra provided the complete picture, confirmed the low plasma temperature measured in February 2005, and showed that V1118 Ori's X-ray flux was strongly correlated with the optical and infrared fluxes, although intrinsic variability (because of magnetic activity) was also observed. Compared to the 2002 flux, the increase in X-ray flux was not large during the outburst and the post-outburst X-ray observation in late 2007 showed a lower flux level than observed serendipitously in 2002, which could either indicate that the outburst significantly impacted the stellar magnetosphere to leave it in a lower state than before the outburst, or that the 2002 observation caught V1118 Ori in a quiescent high state. The X-ray spectra showed evidence of thermal variations of the corona during the outburst, but the signal-to-noise ratio of our data was not high (except in March 2006 during which we could detect a second temperature component, probably due to the increase in flux at the end of the observation, likely contaminated by a flare). After the apparent change to a cooler plasma temperature in the early phase of the outburst, we argue that V1118 Ori's corona returned to its "normal", hotter state before the end of the outburst, but that the continuing mass infall affected significantly the magnetospheric configuration.

Our observations have shown the interplay between disk and stellar magnetosphere in young accreting stars, as demonstrated by the changes in the X-ray plasma emission caused by the strong increase in mass infall from the disk onto the stellar photosphere. While our data do not directly help to determine the origin of the outbursts (thermal disk instabilities or instabilities because of close companions or giant planets in the disk), it is interesting that Reipurth et al. (2007) identified V1118 Ori as a close visual binary. Future observations should, ideally, take this binarity into account and determine which star is actually outbursting.

Acknowledgements. We acknowledge support by NASA through Chandra award DD5-6029X and through XMM-Newton award NNG05GI96G to Columbia University, SAO GO6-7005D, SAO GO8-9020X, and NNX07AI37G to the University of Colorado. M. A. acknowledges support from a Swiss National Science Foundation Professorship (PP002-110504). G. S. Stringfellow further acknowledged support from NASA programs NNX06AG44G, NNX07AT30G, and Spitzer through JPL contract 1265288. The Chandra X-ray Observatory Center is operated by the Smithsonian Astrophysical Observatory for and on behalf of NASA under contract NAS8-03060. Based on observations obtained with XMM-Newton, an ESA science mission with instruments and contributions directly funded by ESA Member States and NASA. This work is based in part on observations made with the Spitzer Space Telescope, which is operated by the Jet Propulsion Laboratory, California Institute of Technology under a contract with NASA. Support for this work was provided by NASA. Stony Brook's participation in SMARTS is made possible by support from the offices of the Provost and the Vice President for Research. We thank J. Allyn Smith, P. McGehee, J. Espinoza, and D. Gonzalez for doing the observations with the SMARTS telescopes. We also thank H. Tannanbaum and N. Schartel for granting Chandra DDT and XMM-Newton TOO time to observe V1118 Ori. We also thank N. Grosso for discussions of quantiles and outbursting sources, A. Carmona and F. Fontani for useful comments on the manuscript. Finally, we thank the referee, D. Lorenzetti, for comments that clarified aspects of the manuscript.

\section{References}

Ábrahám, P., Juhász, A., Dullemond, C. P., et al. 2009, Nature, 459, 224 Ali, B., \& Depoy, D.L. 1995, AJ, 109, 709

Allen, L. E., Calvet, N., D’Alessio, P., et al. 2004, ApJS, 154, 363

Argiroffi, C., Maggio, A., \& Peres, G. 2007, A\&A, 465, L5

Armitage, P. J., Livio, M., \& Pringle, J. E. 2001, MNRAS, 324, 705

Arnaud, K. A. 1996, in Astronomical Data Analysis Software and Systems V,

ed. G. Jacoby, \& J. Barnes (San Francisco: ASP), ASP Conf. Ser., 101, 1

Aspin, C. 2008, IAU Circ, 8969

Aspin, C., Beck, T. L., \& Reipurth, B. 2008, AJ, 135, 423

Aspin, C., Reipurth, B., Beck, T. L., et al. 2009, ApJ, 692, L67

Audard, M., Skinner, S. L., Smith, K. W., et al. 2005a, in Proc. 13th Cool Stars

Workshop, ed. F. Favata et al. (ESA), 411

Audard, M., Güdel, M., Skinner, S. L., et al. 2005b, ApJ, 635, L81

Bell, K. R., \& Lin, D. N. C. 1994, ApJ, 427, 987

Bessell, M. S., \& Brett, J. M. 1988, PASP, 100, 1134

Bonnell, I., \& Bastien, P. 1992, ApJ, 401, L31

Briceño, C., Vivas, A. K., Hernández, J., et al. 2004, ApJ, 606, L123

Calvet, N., \& Gullbring, E. 1998, ApJ, 509, 802

Carr, J. S. 1989, ApJ, 345, 522

Chandler, C. J., Carlstrom, J. E., \& Scoville, N. Z. 1995, ApJ, 446, 793

Clarke, C. J., \& Syer, D. 1996, MNRAS, 278, L23

Drake, J. J. 2005, 13th Cambridge Workshop on Cool Stars, Stellar Systems and the Sun, 560, 519

Edwards, S. 2009, AIP Conf. Ser., 1094, 29

Fazio, G. G., Hora, J. L., Allen, L. E., et al. 2004, ApJS, 154, 10

Fitzpatrick, E. L. 1999, PASP, 111, 63

Garcia, J. G., \& Parsamian, E. S. 2000, Informational Bulletin on Variable Stars, 4925,1

Garcia, J. G., \& Parsamian, E. S. 2008, Information Bulletin on Variable Stars, 5829,1

Garcia, J. G., Parsamian, E. S., \& Velazquez, J. C. 2006, Information Bulletin on Variable Stars, 5691, 1

Grevesse, N., \& Sauval, A. J. 1998, Space Sci. Rev., 85, 161

Grosso, N., Kastner, J. H., Ozawa, H., et al. 2005, A\&A, 438, 159

Güdel, M., \& Telleschi, A. 2007, A\&A, 474, L25

Güdel, M., Skinner, S. L., Briggs, K. R., et al. 2005, ApJ, 626, L53

Güdel, M., Skinner, S. L., Audard, M., et al. 2008, A\&A, 478, 797

Gullbring, E., Hartmann, L., Briceño, C., \& Calvet, N. 1998, ApJ, 492, 323

Günther, H. M., \& Schmitt, J. H. M. M. 2009, A\&A, 494, 1041

Günther, H. M., Liefke, C., Schmitt, J. H. M. M., et al. 2006, A\&A, 459, L29

Günther, H. M., Schmitt, J. H. M. M., Robrade, J., \& Liefke, C. 2007, A\&A, 466, 1111

Hartigan, P., Edwards, S., \& Ghandour, L. 1995, ApJ, 452, 736

Hartmann, L., \& Kenyon, S. J. 1996, ARA\&A, 34, 207

Hartmann, L., Kenyon, S., \& Hartigan, P. 1993, Protostars and Planets III, 497

Hartmann, L., Calvet, N., Gullbring, E., \& D’Alessio, P. 1998, ApJ, 495, 385

Hauschildt, P. H., Allard, F., \& Baron, E. 1999, ApJ 512, 377

Herbig, G. H. 2008, AJ, 135, 637

Hillenbrand, L. A. 1997, AJ, 113, 1733

Hillenbrand, L. A., Strom, S. E., Calvet, N., et al. 1998, AJ, 116, 1816

Hong, J., Schlegel, E. M., \& Grindlay, J. E. 2004, ApJ, 614, 508

Houck, J. R., Roellig, T. L., van Cleve, J., et al. 2004, ApJS, 154, 18

Itagaki, K. 2008, IAU Circ., 8968,

Jansen, F., Lumb, D., Altieri, B., et al. 2001, A\&A, 365, L1

Jones, A. F. A. L. 2008a, Central Bureau Electronic Telegrams, 1217, 1

Jones, A. F. A. L. 2008b, Central Bureau Electronic Telegrams, 1231, 1

Kastner, J. H., Huenemoerder, D. P., Schulz, N. S., et al. 2002, ApJ, 567, 434

Kastner, J. H., Richmond, M., Grosso, N., et al. 2004, Nature, 430, 429

Kastner, J. H., Franz, G., Grosso, N., et al. 2005, ApJS, 160, 511

Kastner, J. H., Richmond, M., Grosso, N., et al. 2006a, ApJ, 648, L43 
Kastner, J. H., Richmond, M., Simon, T., et al. 2006b, Central Bureau Electronic Telegrams, 760, 1

Kenyon, S. J., \& Hartmann, L. 1987, ApJ, 323, 714

Kenyon, S. J., Hartmann, L., \& Hewett, R. 1988, ApJ, 325, 231

Kenyon, S. J., Calvet, N., \& Hartmann, L. 1993, ApJ, 414, 676

Kessler-Silacci, J. E., Hillenbrand, L. A., Blake, G. A., \& Meyer, M. R. 2005, ApJ, 622, 404

Kessler-Silacci, J. E., Augereau, J.-C., Dullemond, C. P., et al. 2006, ApJ, 639, 275

Kóspál, Á., Nemeth, P., Ábrahám, P., et al. 2008, Information Bulletin on Variable Stars, 5819, 1

Lin, D. N. C., \& Papaloizou, J. 1985, Protostars and Planets II, 981

Lodato, G., \& Clarke, C. J. 2004, MNRAS, 353, 841

Lorenzetti, D., Giannini, T., Calzoletti, L., et al. 2006, A\&A, 453, 579

Lorenzetti, D., Giannini, T., Larionov, V. M., et al. 2007, ApJ, 665, 1182

Lorenzetti, D., Larionov, V. M., Giannini, T., et al. 2009, ApJ, 693, 1056

Maggio, A., Flaccomio, E., Favata, F., et al. 2007, ApJ, 660, 1462

Meyer, M. R., Calvet, N., \& Hillenbrand, L. A. 1997, AJ, 114, 288

McGehee, P. M., Smith, J. A., Henden, A. A., et al. 2004, ApJ, 616, 1058

McNeil, J. W. 2004, IAU Circ., 616, 8284

Muench, A., Getman, K., Hillenbrand, L., \& Preibisch, T. 2008, Handbook of Star Forming Regions, Volume I: The Northern Sky, ASP Monograph Publications, Vol. 4, ed. B. Reipurth, 483

Ness, J.-U., \& Schmitt, J. H. M. M. 2005, A\&A, 444, L41

Pfalzner, S., Tackenberg, J., \& Steinhausen, M. 2008, A\&A, 487, L45

Preibisch, T., Kim, Y.-C., Favata, F., et al. 2005, ApJS, 160, 401

Ramírez, S. V., Rebull, L., Stauffer, J., et al. 2004, AJ, 128, 787

Reipurth, B., Guimarães, M. M., Connelley, M. S., \& Bally, J. 2007, AJ, 134, 2272

Rieke, G. H., Lebofsky, M. J., \& Low, F. J. 1985, AJ, 90, 900

Rieke, G. H., Young, E. T., Engelbracht, C. W., et al. 2004, ApJS, 154, 25
Robrade, J., \& Schmitt, J. H. M. M. 2006, A\&A, 449, 737

Robrade, J., \& Schmitt, J. H. M. M. 2007, A\&A, 473, 229

Robitaille, T., Whitney, B. A., Indebetouw, R., \& Wood, K. 2007, ApJS, 169, 328

Sacco, G. G., Argiroffi, C., Orlando, S., et al. 2008, A\&A, 491, L17

Schmitt, J. H. M. M., Robrade, J., Ness, J.-U., et al. 2005, A\&A, 432, L35

Schneider, P. C., \& Schmitt, J. H. M. M. 2008, A\&A, 488, L13

Skinner, S. L., Briggs, K. R., \& Güdel, M. 2006, ApJ, 643, 995

Skinner, S. L., Sokal, K. R., Güdel, M., \& Briggs, K. R. 2009, ApJ, 696, 766

Skinner, S. L., Sokal, K. R., Megeath, S. T., et al. 2009, ApJ, 701, 710

Smith, K. W., Audard, M., Güdel, M., et al. 2005, in Proc. 13th Cool Stars Workshop, ed. F. Favata et al. (ESA), 971

Smith, R. K., Brickhouse, N. S., Liedahl, D. A., \& Raymond, J. C. 2001, ApJ, 556, L91

Stassun, K. G., Mathieu, R. D., Mazeh, T., \& Vrba, F. J. 1999, AJ, 117, 2941

Stelzer, B., \& Schmitt, J. H. M. M. 2004, A\&A, 418, 687

Stelzer, B., Hubrig, S., Orlando, S., et al. 2009, A\&A, 499, 529

Strüder, L., Aschenbach, B., Bräuniger, H., et al. 2001, A\&A, 365, L18

Telleschi, A., Güdel, M., Briggs, K. R., et al. 2007, A\&A, 468, 443

Turner, M. J. L., Abbey, A., Arnaud, M., et al. 2001, A\&A, 365, L26

Venkat, V., \& Anandarao, B. G. 2008, CBET, 1596

Vorobyov, E. I., \& Basu, S. 2005, ApJ, 633, L137

Vorobyov, E. I., \& Basu, S. 2006, ApJ, 650, 956

Weisskopf, M. C., O’dell, S. L., \& van Speybroeck, L. P. 1996, Proc. SPIE, 2805, 2

Werner, M. W., Roellig, T. L., Low, F. J., et al. 2004, ApJS, 154, 1

Whitney, B. A., Wood, K., Bjorkman, J. E., \& Wolff, M. J. 2003a, ApJ, 591, 1049

Whitney, B. A., Wood, K., Bjorkman, J. E., \& Cohen, M. 2003b, ApJ, 598, 1079 Williams, P., Bembrick, C., \& Lee, S. 2005, IAUC, 8460, 3

Wood, K., Wolff, M. J., Bjorkman, J. E., \& Whitney, B. 2002, ApJ, 564, 887

Pages 20 to 26 are available in the electronic edition of the journal at http : //www . aanda . org 


\section{Appendix A: Details on the SMARTS data reduction}

The ANDICAM optical detector is a Fairchild $4472048 \times$ 2048 CCD with $30 \mu \mathrm{m}$ pixels. It was used in $2 \times 2$ binning mode, yielding a nominal plate scale of 0.369 arcsec pixel $^{-1}$ and approximately a $6^{\prime} \times 6^{\prime}$ field. Readnoise was $6.5 \mathrm{e}^{-} \mathrm{rms}$; gain is $2.3 \mathrm{e}^{-} / \mathrm{DN}$. It was read with a single amplifier. Filters were Johnson $B$ and $V$ and Kron-Cousins $R$ and $I$. We generally took 3 exposures in $B$ and $V$, and 2 images in $R_{\mathrm{C}}$ and $I_{\mathrm{C}}$, and co-added these prior to analysis. The IR channel detector is a Rockwell $1024^{2} \mathrm{HgCdTe}$ "Hawaii" array with $18 \mu \mathrm{m}$ pixels binned $2 \times 2$ to yield a 0.274 pixel $^{-1}$ plate scale. The IR channel field is $2 ! 4 \times 2 ! 4$. The used filters are standard CIT/CTIO $J H K$. We flattened the images using dome flats obtained every 3 nights. We observed using a 3-point dither pattern and a $40^{\prime \prime}$ throw, and used the median of the unshifted images as the local sky.

\section{A.1. Differential photometry}

We initially determined relative magnitudes using differential photometry. In the optical, we selected the 11 brightest stars in the $\approx 6^{\prime}$ field of view (Table A.1; V372 Ori was overexposed). Instrumental magnitudes were determined for the target and all the comparison stars, by summing the counts in a 5 pixel (1."85) aperture. Background was the median level in an annulus from 10-20 pixel (3". $69-77^{\prime \prime} .38$ ) radius. Since V1118 Ori is in a region of complex nebulosity, we also tried modeling the background by taking radial cuts through the image and extrapolating the level at the position of the target. This affected the magnitudes at less than $5 \%$ at $B$, and even less at longer wavelengths.

In the near-infrared, we used 6 comparison stars in the field (again excluding V372 Ori). We used a 5 pixel (1".37) aperture. Background was the median level in an annulus from 10-20 pixel (2'.74-5'.48) radius.

We constructed a single comparison, the "superstar" from a weighted sum of all the comparison stars. The differential magnitude was the difference between the instrumental magnitudes of the target and the "superstar".

Most of the stars in this field are known or suspected variables. Fortunately, none were as variable as V1118 Ori. We assumed that, by summing all the comparisons, the "superstar" would be less variable than any single comparison. We constructed light curves using subsets of the comparison stars, and did not see any gross effects that could be attributed to variations of the comparisons. Nonetheless we did not incorporate those stars with measured standard deviations after absolute calibration (see below) $>0.10$ mag at $V$ into the optical "superstar". We caution that there is no certainty that the comparison was stable at the few percent level.

\section{A.2. Optical absolute calibration}

Although the SMARTS operations are not optimized for absolute photometry, single observations are made of Landolt standard fields on each night judged photometric to establish the photometric zero point. These can be used to determine apparent magnitudes with an accuracy of better than $10 \%$.

On each night on which we had both observations of the target and of a photometric standard field, we computed the zero-point offset (apparent magnitude - instrumental magnitude). We assumed the standard atmospheric extinction correction for Cerro Tololo. Since the standard field was observed only once per night, we could not account for changes in transparency through the night. We did not solve for reddening terms, so the photometric solution consisted of only the zero-point.

We used the zero-point correction to determine the apparent magnitudes of all the comparison stars in the field. We could do this on 71 nights. In doing so, we confirmed that all the comparison stars were variable (Table A.1). We compared these magnitudes to the $V, I_{\mathrm{C}}$ magnitudes published by Hillenbrand (1997). The median offsets were 0.08 and 0.23 mag at $V$ and $I_{C}$, respectively. Given that all the stars are variable members of the Orion Nebula population, this was acceptable agreement.

We applied this absolute calibration on all nights (including non-photometric nights) to determine the apparent magnitudes of the target. There were 14 nights where SMARTS photometry overlapped with the Villanova photometry. On these nights there were systematic offsets, in the sense (SMARTS-Villanova), of $0.16 \pm 0.10,0.24 \pm 0.04$, and $0.34 \pm 0.04 \mathrm{mag}$ in $V, R_{\mathrm{C}}$, and $I_{\mathrm{C}}$, respectively. The cause for the systematic offsets might be the lack of a color term in the photometric solution, exacerbated by the fact that most of our comparisons have $V-I_{\mathrm{C}}$ colors $>2$ mag. To allow comparison between the different telescope data, we applied the above systematic offsets to the SMARTS magnitudes and gave the nightly average data in Table A.2.

\section{A.3. Near-infrared absolute calibration}

For the $J H K$ absolute calibration, we compared the instrumental magnitudes directly to the 2MASS magnitudes of the 6 comparison stars. To check for target variability, we compared these magnitudes to those published in the DENIS catalog, and by Ali \& Depoy (1995) and Hillenbrand et al. (1998). Two of the six comparisons (2MASS J05344159-0534249 and 2MASS J05344219-0533036 appear variable, with variances $>0.2 \mathrm{mag}$ at $K$ (4 observations), and 2 others (JW 94 and JW 100) appeared variable in the optical (Table A.1).

Our ability to absolutely calibrate the photometry was ultimately limited by the stability of the zero-point. By using all the possible comparison stars, on average the zero-point will be more stable than determined from any single comparison star. We did not include the systematic uncertainty in the zero-point in our error budgets. Overall, the $J H K$ fluxes compared well to those published by Lorenzetti et al. (2007). However, we added a systematic correction of $+0.1 \mathrm{mag}$ for $J$ and +0.2 mag for $K$ to match their photometry. V1118 Ori is intrinsically variable, as are the comparison stars. The only reason for applying the systematic offsets to our data was to simplify graphical comparison with the Lorenzetti et al. data. The offsets do not change significantly the results of this paper, they merely shift the near-infrared data points by 0.1 to $0.2 \mathrm{mag}$, e.g., in Figs. 10 and 11 . 
Table A.1. Comparison stars for the SMARTS photometry.

\begin{tabular}{|c|c|c|c|c|c|c|}
\hline Comparison & $B$ & V & $R$ & $I$ & $\delta V$ & $\delta I$ \\
\hline IV Ori & $16.19 \pm 0.35$ & $14.94 \pm 0.26$ & $13.97 \pm 0.22$ & $13.19 \pm 0.13$ & & \\
\hline CSV 6218 & $17.40 \pm 0.13$ & $15.90 \pm 0.13$ & $14.74 \pm 0.13$ & $13.68 \pm 0.15$ & 0.20 & 0.36 \\
\hline V759 Ori & $16.81 \pm 0.12$ & $15.44 \pm 0.09$ & $14.32 \pm 0.10$ & $13.14 \pm 0.13$ & 0.10 & 0.24 \\
\hline NGC 1977311 & $14.32 \pm 0.11$ & $13.24 \pm 0.09$ & $12.46 \pm 0.09$ & $11.73 \pm 0.12$ & & \\
\hline V1175 Ori & $16.59 \pm 0.10$ & $15.19 \pm 0.07$ & $14.19 \pm 0.09$ & $13.26 \pm 0.12$ & 0.08 & 0.14 \\
\hline JW 94 & $20.82 \pm 0.35$ & $19.10 \pm 0.17$ & $17.37 \pm 0.10$ & $15.45 \pm 0.13$ & 0.10 & 0.29 \\
\hline JW 100 & $20.25 \pm 0.32$ & $18.48 \pm 0.15$ & $17.09 \pm 0.10$ & $15.44 \pm 0.12$ & 0.03 & 0.23 \\
\hline Par 1606 & $17.18 \pm 0.10$ & $15.61 \pm 0.07$ & $14.38 \pm 0.08$ & $13.22 \pm 0.12$ & 0.03 & 0.12 \\
\hline CXORRS J053447.9-053544 & $22.00 \pm 0.99$ & $19.79 \pm 0.32$ & $17.76 \pm 0.13$ & $15.79 \pm 0.12$ & 0.67 & 0.23 \\
\hline NSV2246 & $11.32 \pm 0.26$ & $10.30 \pm 0.17$ & $9.78 \pm 0.16$ & $9.25 \pm 0.13$ & 0.08 & 0.06 \\
\hline JW 33 & $18.75 \pm 0.32$ & $17.30 \pm 0.10$ & $16.16 \pm 0.09$ & $14.84 \pm 0.12$ & -0.03 & 0.15 \\
\hline
\end{tabular}

Notes. $\delta V$ and $\delta I$ are the difference between these $V, I$ determinations and those published by Hillenbrand (1997).

Table A.2. SMARTS average nightly magnitudes.

\begin{tabular}{|c|c|c|c|c|c|c|c|c|}
\hline MJD & $U$ & $B$ & $V$ & $R$ & $I$ & $J$ & $H$ & $K$ \\
\hline 53012 & & & & & & $12.74 \pm 0.07$ & $11.68 \pm 0.03$ & $11.15 \pm 0.04$ \\
\hline 53031 & & & & & & $12.72 \pm 0.06$ & $11.38 \pm 0.03$ & $11.06 \pm 0.03$ \\
\hline 53038 & $18.17 \pm 0.05$ & $18.37 \pm 0.03$ & $17.45 \pm 0.02$ & $16.32 \pm 0.01$ & $14.93 \pm 0.01$ & & & \\
\hline 53058 & $18.67 \pm 0.11$ & & & & & & & $11.32 \pm 0.03$ \\
\hline 53062 & $18.41 \pm 0.08$ & $18.41 \pm 0.03$ & $17.61 \pm 0.02$ & $16.32 \pm 0.02$ & $14.91 \pm 0.01$ & & & \\
\hline 53092 & $18.32 \pm 0.07$ & $18.35 \pm 0.03$ & $17.47 \pm 0.02$ & $16.18 \pm 0.01$ & $14.86 \pm 0.01$ & $12.54 \pm 0.07$ & $11.23 \pm 0.03$ & $11.02 \pm 0.04$ \\
\hline 53103 & $17.70 \pm 0.03$ & $17.91 \pm 0.02$ & $17.06 \pm 0.01$ & $15.98 \pm 0.01$ & $14.72 \pm 0.01$ & & & \\
\hline 53381 & & $15.62 \pm 0.01$ & $14.70 \pm 0.01$ & $13.78 \pm 0.01$ & $12.89 \pm 0.01$ & $12.23 \pm 0.05$ & $11.12 \pm 0.03$ & $10.56 \pm 0.03$ \\
\hline 53382 & & $15.68 \pm 0.01$ & $14.78 \pm 0.01$ & $13.87 \pm 0.02$ & $12.93 \pm 0.01$ & $12.27 \pm 0.05$ & $11.17 \pm 0.03$ & $10.64 \pm 0.03$ \\
\hline 53383 & & $15.61 \pm 0.01$ & $14.70 \pm 0.01$ & $13.79 \pm 0.01$ & $12.87 \pm 0.01$ & $11.81 \pm 0.05$ & $11.12 \pm 0.03$ & $10.53 \pm 0.03$ \\
\hline 53384 & & $15.92 \pm 0.05$ & $14.94 \pm 0.02$ & $13.97 \pm 0.02$ & $13.09 \pm 0.03$ & & & \\
\hline 53385 & & $15.25 \pm 0.01$ & $14.30 \pm 0.01$ & $13.40 \pm 0.01$ & $12.56 \pm 0.01$ & $11.71 \pm 0.04$ & $10.86 \pm 0.02$ & $10.35 \pm 0.03$ \\
\hline 53394 & & $14.36 \pm 0.02$ & $13.48 \pm 0.01$ & $12.71 \pm 0.01$ & $11.90 \pm 0.01$ & $11.18 \pm 0.03$ & $10.43 \pm 0.02$ & $9.85 \pm 0.02$ \\
\hline 53395 & & $14.04 \pm 0.02$ & $13.09 \pm 0.01$ & $12.35 \pm 0.01$ & $11.59 \pm 0.01$ & $11.23 \pm 0.03$ & $10.26 \pm 0.02$ & $10.0 \pm 0.02$ \\
\hline 53396 & & $14.32 \pm 0.02$ & $13.41 \pm 0.01$ & $12.59 \pm 0.01$ & $11.80 \pm 0.01$ & $11.07 \pm 0.03$ & $10.30 \pm 0.02$ & $9.77 \pm 0.02$ \\
\hline 53397 & & $14.33 \pm 0.01$ & $13.40 \pm 0.01$ & $12.60 \pm 0.01$ & $11.81 \pm 0.01$ & $11.41 \pm 0.03$ & $10.41 \pm 0.02$ & $9.94 \pm 0.02$ \\
\hline 53398 & & $14.51 \pm 0.01$ & $13.58 \pm 0.01$ & $12.76 \pm 0.01$ & $11.96 \pm 0.01$ & $11.23 \pm 0.03$ & $10.41 \pm 0.02$ & $9.97 \pm 0.02$ \\
\hline 53399 & & $14.44 \pm 0.01$ & $13.52 \pm 0.01$ & $12.70 \pm 0.01$ & $11.91 \pm 0.01$ & $11.39 \pm 0.03$ & $10.58 \pm 0.02$ & $10.01 \pm 0.02$ \\
\hline 53400 & & $14.66 \pm 0.02$ & $13.75 \pm 0.01$ & $12.95 \pm 0.01$ & $12.07 \pm 0.01$ & $11.81 \pm 0.04$ & $10.60 \pm 0.02$ & $9.90 \pm 0.03$ \\
\hline 53401 & & $14.31 \pm 0.05$ & $13.42 \pm 0.02$ & $12.60 \pm 0.02$ & $11.90 \pm 0.02$ & $11.25 \pm 0.05$ & $10.17 \pm 0.03$ & $9.78 \pm 0.03$ \\
\hline 53405 & & $14.73 \pm 0.02$ & $13.75 \pm 0.02$ & $12.94 \pm 0.01$ & $12.12 \pm 0.01$ & $11.72 \pm 0.04$ & $10.64 \pm 0.02$ & $10.09 \pm 0.03$ \\
\hline 53407 & & $14.24 \pm 0.01$ & $13.29 \pm 0.01$ & $12.51 \pm 0.01$ & $11.74 \pm 0.01$ & $11.14 \pm 0.03$ & $10.32 \pm 0.02$ & $9.87 \pm 0.02$ \\
\hline 53408 & & $14.21 \pm 0.02$ & $13.35 \pm 0.02$ & $12.59 \pm 0.01$ & $11.84 \pm 0.01$ & $11.17 \pm 0.04$ & $10.41 \pm 0.02$ & $9.90 \pm 0.03$ \\
\hline 53411 & & $14.31 \pm 0.02$ & $13.38 \pm 0.02$ & $12.60 \pm 0.01$ & $11.79 \pm 0.01$ & $11.21 \pm 0.03$ & $10.39 \pm 0.02$ & $10.01 \pm 0.03$ \\
\hline 53413 & & $14.30 \pm 0.02$ & $13.32 \pm 0.02$ & $12.50 \pm 0.01$ & $11.69 \pm 0.01$ & $11.23 \pm 0.03$ & $10.34 \pm 0.02$ & $9.92 \pm 0.03$ \\
\hline 53414 & & $14.24 \pm 0.02$ & $13.38 \pm 0.02$ & $12.61 \pm 0.01$ & $11.81 \pm 0.01$ & $11.06 \pm 0.03$ & $10.38 \pm 0.02$ & $9.93 \pm 0.03$ \\
\hline 53416 & & $14.17 \pm 0.03$ & $13.47 \pm 0.01$ & $12.71 \pm 0.01$ & $11.95 \pm 0.01$ & & & \\
\hline 53417 & & $14.09 \pm 0.03$ & $13.25 \pm 0.01$ & $12.52 \pm 0.01$ & $11.77 \pm 0.01$ & & & \\
\hline 53418 & & $14.08 \pm 0.03$ & $13.25 \pm 0.01$ & $12.49 \pm 0.01$ & $11.75 \pm 0.01$ & & & \\
\hline 53419 & & $14.25 \pm 0.03$ & & $12.65 \pm 0.01$ & $11.87 \pm 0.01$ & & & \\
\hline 53420 & & $14.24 \pm 0.03$ & $13.42 \pm 0.01$ & $12.64 \pm 0.01$ & $11.89 \pm 0.01$ & & & \\
\hline 53421 & & $13.77 \pm 0.04$ & $13.04 \pm 0.02$ & $12.31 \pm 0.01$ & $11.60 \pm 0.01$ & $10.92 \pm 0.03$ & $10.21 \pm 0.02$ & $9.68 \pm 0.03$ \\
\hline 53422 & & $13.78 \pm 0.03$ & $12.88 \pm 0.03$ & $12.15 \pm 0.01$ & $11.42 \pm 0.01$ & $10.69 \pm 0.03$ & $10.04 \pm 0.02$ & $9.58 \pm 0.03$ \\
\hline 53423 & & $13.85 \pm 0.11$ & $13.07 \pm 0.05$ & $12.27 \pm 0.04$ & $11.58 \pm 0.03$ & & & \\
\hline 53424 & & $4.10 \pm 0.05$ & $13.33 \pm 0.02$ & $12.54 \pm 0.01$ & $11.76 \pm 0.01$ & & & \\
\hline 53425 & & $.90 \pm 0.05$ & $13.14 \pm 0.02$ & $12.39 \pm 0.01$ & $11.69 \pm 0$ & & & \\
\hline 53426 & & $13.85 \pm 0.05$ & $13.13 \pm 0.02$ & $12.39 \pm 0.01$ & $11.67 \pm 0.01$ & & & \\
\hline 53427 & & $13.84 \pm 0.04$ & $13.22 \pm 0.01$ & $12.48 \pm 0.01$ & $11.75 \pm 0.01$ & & & \\
\hline 53428 & & $13.55 \pm 0.06$ & $12.95 \pm 0.03$ & $12.24 \pm 0.02$ & $11.55 \pm 0.01$ & $11.18 \pm 0.03$ & $10.23 \pm 0.01$ & $9.67 \pm 0.03$ \\
\hline 53429 & & $14.05 \pm 0.03$ & $13.29 \pm 0.01$ & $12.54 \pm 0.01$ & $11.78 \pm 0.01$ & & & \\
\hline 53430 & & $13.83 \pm 0.02$ & $13.06 \pm 0.02$ & $12.31 \pm 0.01$ & $11.60 \pm 0.01$ & $11.23 \pm 0.03$ & $10.20 \pm 0.01$ & $9.80 \pm 0.02$ \\
\hline 53431 & & $13.89 \pm 0.02$ & $12.97 \pm 0.01$ & $12.24 \pm 0.01$ & $11.55 \pm 0.01$ & & & \\
\hline 53432 & & $13.74 \pm 0.03$ & $13.01 \pm 0.01$ & $12.27 \pm 0.01$ & $11.54 \pm 0.01$ & & & \\
\hline 53433 & & $13.60 \pm 0.02$ & $12.84 \pm 0.01$ & $12.11 \pm 0.01$ & $11.41 \pm 0.01$ & & & \\
\hline 53458 & & $13.83 \pm 0.03$ & $12.90 \pm 0.02$ & $12.15 \pm 0.01$ & $11.41 \pm 0.01$ & $10.74 \pm 0.03$ & $9.83 \pm 0.01$ & $9.38 \pm 0.02$ \\
\hline 53460 & & $13.95 \pm 0.02$ & $13.03 \pm 0.02$ & $12.26 \pm 0.01$ & $11.49 \pm 0.01$ & $10.85 \pm 0.03$ & $10.04 \pm 0.01$ & $9.39 \pm 0.02$ \\
\hline 53463 & & $13.95 \pm 0.03$ & $13.01 \pm 0.03$ & $12.21 \pm 0.01$ & $11.45 \pm 0.01$ & $10.67 \pm 0.03$ & $9.98 \pm 0.01$ & $9.30 \pm 0.02$ \\
\hline 53464 & & $13.99 \pm 0.02$ & $13.04 \pm 0.02$ & $12.27 \pm 0.01$ & $11.51 \pm 0.01$ & $10.60 \pm 0.03$ & $9.93 \pm 0.01$ & $9.43 \pm 0.02$ \\
\hline
\end{tabular}


A\&A 511, A63 (2010)

Table A.2. continued

\begin{tabular}{|c|c|c|c|c|c|c|c|c|}
\hline MJD & $U$ & $B$ & V & $R$ & $I$ & $J$ & $H$ & $K$ \\
\hline 53467 & & $13.67 \pm 0.02$ & $12.79 \pm 0.02$ & $12.03 \pm 0.01$ & $11.31 \pm 0.01$ & $10.60 \pm 0.03$ & $9.81 \pm 0.01$ & $9.29 \pm 0.02$ \\
\hline 53468 & & $13.81 \pm 0.02$ & $12.90 \pm 0.02$ & $12.12 \pm 0.01$ & $11.38 \pm 0.01$ & $10.80 \pm 0.03$ & $9.81 \pm 0.01$ & $9.30 \pm 0.02$ \\
\hline 53469 & & $13.95 \pm 0.02$ & & & & & $9.87 \pm 0.01$ & \\
\hline 53470 & & & $13.00 \pm 0.02$ & $12.19 \pm 0.01$ & $11.46 \pm 0.01$ & $10.90 \pm 0.03$ & & $9.20 \pm 0.02$ \\
\hline 53476 & & $14.17 \pm 0.03$ & $13.23 \pm 0.02$ & $12.48 \pm 0.01$ & $11.69 \pm 0.01$ & $10.87 \pm 0.03$ & $10.08 \pm 0.02$ & $9.50 \pm 0.03$ \\
\hline 53477 & & $14.22 \pm 0.03$ & $13.25 \pm 0.02$ & $12.48 \pm 0.01$ & $11.67 \pm 0.01$ & $10.81 \pm 0.03$ & $9.97 \pm 0.02$ & $9.51 \pm 0.02$ \\
\hline 53488 & & $13.86 \pm 0.03$ & $12.94 \pm 0.02$ & $12.18 \pm 0.01$ & $11.42 \pm 0.01$ & $10.45 \pm 0.03$ & $9.84 \pm 0.01$ & $9.42 \pm 0.02$ \\
\hline 53498 & & $13.94 \pm 0.14$ & $13.22 \pm 0.04$ & $12.25 \pm 0.02$ & $11.46 \pm 0.01$ & $10.77 \pm 0.03$ & $10.25 \pm 0.01$ & $9.38 \pm 0.02$ \\
\hline 53502 & & & $13.08 \pm 0.06$ & & & & & $9.11 \pm 0.02$ \\
\hline 53584 & & $14.28 \pm 0.17$ & $13.22 \pm 0.01$ & \pm 0.01 & $11.61 \pm 0.01$ & $10.82 \pm 0.08$ & $9.98 \pm 0.02$ & \\
\hline 53608 & & $14.46 \pm 0.01$ & $13.49 \pm 0.01$ & $12.67 \pm 0.01$ & $11.86 \pm 0.01$ & $10.93 \pm 0.05$ & $10.13 \pm 0.02$ & $9.57 \pm 0.03$ \\
\hline 53618 & & $14.43 \pm 0.01$ & $13.44 \pm 0.01$ & $12.62 \pm 0.01$ & $11.78 \pm 0.01$ & $11.01 \pm 0.06$ & $10.21 \pm 0.02$ & $9.57 \pm 0.03$ \\
\hline 53625 & & $14.89 \pm 0.01$ & $13.94 \pm 0.01$ & $13.09 \pm 0.01$ & $12.21 \pm 0.01$ & $11.44 \pm 0.04$ & $10.44 \pm 0.02$ & $9.83 \pm 0.03$ \\
\hline 53632 & & $14.50 \pm 0.02$ & $13.51 \pm 0.01$ & $12.72 \pm 0.01$ & $11.86 \pm 0.01$ & $11.05 \pm 0.05$ & $10.20 \pm 0.02$ & $9.51 \pm 0.03$ \\
\hline 53637 & & $14.62 \pm 0.02$ & $13.54 \pm 0.04$ & & & & $10.24 \pm 0.03$ & $9.30 \pm 0.07$ \\
\hline 53640 & & $14.06 \pm 0.01$ & $13.09 \pm 0.01$ & $12.31 \pm 0.01$ & $11.52 \pm 0.01$ & $11.01 \pm 0.04$ & $10.02 \pm 0.02$ & $9.46 \pm 0.03$ \\
\hline 53650 & & $14.03 \pm 0.01$ & $13.12 \pm 0.01$ & $12.31 \pm 0.01$ & $11.56 \pm 0.01$ & $10.74 \pm 0.04$ & $10.04 \pm 0.02$ & $9.43 \pm 0.03$ \\
\hline 53653 & & $14.34 \pm 0.01$ & $13.43 \pm 0.01$ & $12.62 \pm 0.01$ & $11.77 \pm 0.01$ & $11.39 \pm 0.04$ & $10.48 \pm 0.02$ & $10.02 \pm 0.03$ \\
\hline 53657 & & $14.15 \pm 0.01$ & $13.25 \pm 0.01$ & $12.45 \pm 0.01$ & $11.65 \pm 0.01$ & $10.93 \pm 0.04$ & $10.12 \pm 0.01$ & $9.46 \pm 0.02$ \\
\hline 53663 & & $14.67 \pm 0.01$ & $13.73 \pm 0.01$ & $12.88 \pm 0.01$ & $12.02 \pm 0.01$ & $11.23 \pm 0.04$ & $10.36 \pm 0.02$ & $9.66 \pm 0.03$ \\
\hline 53670 & & $14.76 \pm 0.01$ & $13.82 \pm 0.01$ & $13.01 \pm 0.01$ & $12.16 \pm 0.01$ & $11.73 \pm 0.05$ & $10.86 \pm 0.02$ & $10.14 \pm 0.03$ \\
\hline 53674 & & $14.35 \pm 0.01$ & $13.38 \pm 0.01$ & $12.60 \pm 0.01$ & $11.83 \pm 0.01$ & $11.05 \pm 0.04$ & $10.18 \pm 0.02$ & $9.47 \pm 0.03$ \\
\hline 53678 & & $14.65 \pm 0.01$ & $13.70 \pm 0.01$ & $12.86 \pm 0.01$ & $12.02 \pm 0.01$ & $11.30 \pm 0.04$ & $10.30 \pm 0.02$ & $9.74 \pm 0.03$ \\
\hline 53687 & & $14.45 \pm 0.01$ & $13.52 \pm 0.01$ & $12.69 \pm 0.01$ & $11.87 \pm 0.01$ & $11.05 \pm 0.04$ & $10.26 \pm 0.02$ & $9.57 \pm 0.03$ \\
\hline 53693 & & $14.54 \pm 0.01$ & $13.63 \pm 0.01$ & $12.81 \pm 0.01$ & $12.00 \pm 0.01$ & $11.21 \pm 0.04$ & $10.33 \pm 0.02$ & $9.63 \pm 0.03$ \\
\hline 53700 & & $14.63 \pm 0.01$ & $13.70 \pm 0.01$ & $12.87 \pm 0.01$ & $12.05 \pm 0.01$ & $11.27 \pm 0.04$ & $10.41 \pm 0.02$ & $9.69 \pm 0.03$ \\
\hline 53705 & & $14.96 \pm 0.01$ & $13.98 \pm 0.01$ & $13.13 \pm 0.01$ & $12.27 \pm 0.01$ & $11.44 \pm 0.05$ & $10.53 \pm 0.02$ & $9.81 \pm 0.03$ \\
\hline 53710 & & $15.35 \pm 0.01$ & $14.51 \pm 0.01$ & & $12.75 \pm 0.01$ & $11.75 \pm 0.07$ & $10.95 \pm 0.02$ & $10.24 \pm 0.03$ \\
\hline 53712 & & $15.64 \pm 0.01$ & $14.74 \pm 0.01$ & $13.81 \pm 0.01$ & $12.89 \pm 0.01$ & & $10.91 \pm 0.02$ & \\
\hline 53714 & & $15.50 \pm 0.01$ & $14.63 \pm 0.01$ & $13.73 \pm 0.01$ & $12.84 \pm 0.01$ & $11.84 \pm 0.06$ & $10.89 \pm 0.02$ & $10.08 \pm 0.04$ \\
\hline 53715 & & $15.43 \pm 0.01$ & $14.53 \pm 0.01$ & $13.66 \pm 0.01$ & $12.79 \pm 0.01$ & $11.80 \pm 0.05$ & $10.85 \pm 0.02$ & $9.97 \pm 0.03$ \\
\hline 53716 & & $15.69 \pm 0.01$ & $14.77 \pm 0.01$ & $13.85 \pm 0.01$ & $12.93 \pm 0.01$ & $11.95 \pm 0.06$ & $10.93 \pm 0.02$ & $10.12 \pm 0.04$ \\
\hline 53717 & & $15.54 \pm 0.01$ & $14.61 \pm 0.01$ & $13.68 \pm 0.01$ & $12.76 \pm 0.01$ & $11.84 \pm 0.06$ & $10.87 \pm 0.02$ & $10.08 \pm 0.04$ \\
\hline 53718 & & $15.36 \pm 0.01$ & $14.44 \pm 0.01$ & $13.54 \pm 0.01$ & $12.65 \pm 0.01$ & $11.72 \pm 0.05$ & $10.80 \pm 0.02$ & $9.95 \pm 0.03$ \\
\hline 53719 & & $15.08 \pm 0.02$ & $14.14 \pm 0.01$ & $13.26 \pm 0.01$ & $12.38 \pm 0.01$ & $11.48 \pm 0.05$ & $10.60 \pm 0.02$ & $9.83 \pm 0.03$ \\
\hline 53720 & & $15.54 \pm 0.02$ & $14.63 \pm 0.01$ & $13.69 \pm 0.01$ & $12.75 \pm 0.01$ & $11.87 \pm 0.05$ & $10.94 \pm 0.02$ & $10.22 \pm 0.03$ \\
\hline 53721 & & $15.36 \pm 0.02$ & $14.41 \pm 0.01$ & $13.49 \pm 0.01$ & $12.61 \pm 0.01$ & $11.73 \pm 0.05$ & $10.78 \pm 0.02$ & $10.06 \pm 0.03$ \\
\hline 53722 & & $15.25 \pm 0.01$ & $14.30 \pm 0.01$ & $13.42 \pm 0.01$ & $12.54 \pm 0.01$ & $11.69 \pm 0.06$ & $10.73 \pm 0.02$ & $10.07 \pm 0.04$ \\
\hline 53723 & & $14.97 \pm 0.01$ & $14.03 \pm 0.01$ & $13.20 \pm 0.01$ & $12.37 \pm 0.01$ & $11.50 \pm 0.05$ & $10.66 \pm 0.02$ & $9.93 \pm 0.03$ \\
\hline 53724 & & $14.96 \pm 0.01$ & $14.03 \pm 0.01$ & $13.16 \pm 0.01$ & $12.35 \pm 0.01$ & $11.51 \pm 0.05$ & $10.64 \pm 0.02$ & $9.89 \pm 0.03$ \\
\hline 53725 & & $14.84 \pm 0.01$ & $13.91 \pm 0.01$ & $13.06 \pm 0.01$ & $12.26 \pm 0.01$ & $11.36 \pm 0.05$ & $10.57 \pm 0.02$ & $9.83 \pm 0.03$ \\
\hline 53727 & & $15.00 \pm 0.01$ & $14.07 \pm 0.01$ & $13.23 \pm 0.01$ & $12.41 \pm 0.01$ & $11.49 \pm 0.05$ & $10.66 \pm 0.02$ & $9.90 \pm 0.03$ \\
\hline 53728 & & $15.00 \pm 0.01$ & $14.03 \pm 0.01$ & $13.17 \pm 0.01$ & $12.32 \pm 0.01$ & $11.54 \pm 0.05$ & $10.62 \pm 0.02$ & $9.93 \pm 0.03$ \\
\hline 53748 & & $16.01 \pm 0.02$ & $15.16 \pm 0.02$ & $14.26 \pm 0.01$ & $13.35 \pm 0.01$ & $12.15 \pm 0.07$ & $11.08 \pm 0.03$ & $10.36 \pm 0.04$ \\
\hline 53749 & & $16.08 \pm 0.02$ & $15.21 \pm 0.02$ & $14.32 \pm 0.01$ & $13.42 \pm 0.01$ & $12.19 \pm 0.07$ & $11.11 \pm 0.03$ & $10.34 \pm 0.04$ \\
\hline 53750 & & $16.20 \pm 0.02$ & $15.36 \pm 0.02$ & $14.42 \pm 0.01$ & $13.51 \pm 0.01$ & $11.85 \pm 0.08$ & $11.20 \pm 0.03$ & $10.10 \pm 0.04$ \\
\hline 53751 & & $16.16 \pm 0.02$ & $15.26 \pm 0.01$ & $14.31 \pm 0.01$ & $13.37 \pm 0.01$ & $12.19 \pm 0.06$ & $11.04 \pm 0.03$ & $10.22 \pm 0.04$ \\
\hline 53752 & & $16.10 \pm 0.01$ & $15.20 \pm 0.01$ & $14.26 \pm 0.01$ & $13.32 \pm 0.01$ & $12.03 \pm 0.06$ & $11.02 \pm 0.02$ & $10.20 \pm 0.04$ \\
\hline 53755 & & $15.51 \pm 0.01$ & $14.59 \pm 0.01$ & $13.68 \pm 0.01$ & $12.77 \pm 0.01$ & $11.83 \pm 0.06$ & $10.68 \pm 0.02$ & $9.79 \pm 0.03$ \\
\hline 53757 & & $15.68 \pm 0.01$ & $14.73 \pm 0.01$ & $13.79 \pm 0.01$ & $12.85 \pm 0.01$ & $11.94 \pm 0.06$ & $10.81 \pm 0.03$ & $9.94 \pm 0.04$ \\
\hline 53759 & & $15.17 \pm 0.01$ & $14.28 \pm 0.01$ & $13.40 \pm 0.01$ & $12.53 \pm 0.01$ & $11.70 \pm 0.05$ & $10.70 \pm 0.02$ & $9.81 \pm 0.03$ \\
\hline 53762 & & $14.98 \pm 0.01$ & $14.07 \pm 0.01$ & $13.21 \pm 0.01$ & $12.39 \pm 0.01$ & $11.54 \pm 0.05$ & $10.64 \pm 0.02$ & $9.89 \pm 0.03$ \\
\hline 53765 & & $15.48 \pm 0.01$ & $14.64 \pm 0.01$ & $13.73 \pm 0.01$ & $12.86 \pm 0.01$ & $11.77 \pm 0.06$ & $10.91 \pm 0.02$ & $10.18 \pm 0.04$ \\
\hline 53767 & & $15.74 \pm 0.01$ & $14.89 \pm 0.01$ & $13.94 \pm 0.01$ & $13.08 \pm 0.01$ & $11.88 \pm 0.07$ & $11.03 \pm 0.03$ & $10.07 \pm 0.05$ \\
\hline 53770 & & $15.81 \pm 0.01$ & $14.87 \pm 0.01$ & $13.95 \pm 0.01$ & $13.06 \pm 0.01$ & $12.09 \pm 0.06$ & $11.06 \pm 0.03$ & $10.17 \pm 0.04$ \\
\hline 53773 & & $16.00 \pm 0.01$ & $15.11 \pm 0.01$ & $14.17 \pm 0.01$ & $13.24 \pm 0.01$ & $12.12 \pm 0.07$ & $11.03 \pm 0.03$ & $10.21 \pm 0.04$ \\
\hline 53776 & & $15.73 \pm 0.02$ & $14.82 \pm 0.01$ & $13.90 \pm 0.01$ & $12.99 \pm 0.01$ & $11.90 \pm 0.06$ & $10.91 \pm 0.02$ & $10.20 \pm 0.04$ \\
\hline 53779 & & $15.88 \pm 0.02$ & $15.00 \pm 0.01$ & $14.01 \pm 0.01$ & $13.11 \pm 0.01$ & $11.97 \pm 0.07$ & $11.00 \pm 0.03$ & $10.33 \pm 0.04$ \\
\hline 53782 & & $15.71 \pm 0.01$ & $14.81 \pm 0.01$ & $13.84 \pm 0.01$ & $12.98 \pm 0.01$ & $11.79 \pm 0.07$ & $11.01 \pm 0.03$ & $10.30 \pm 0.04$ \\
\hline 53785 & & $15.72 \pm 0.01$ & $14.85 \pm 0.01$ & $13.95 \pm 0.01$ & $13.05 \pm 0.01$ & $11.98 \pm 0.07$ & $10.96 \pm 0.03$ & $10.31 \pm 0.04$ \\
\hline 53789 & & $15.83 \pm 0.01$ & $14.95 \pm 0.01$ & $14.00 \pm 0.01$ & $13.09 \pm 0.01$ & $12.04 \pm 0.06$ & $10.99 \pm 0.03$ & $10.24 \pm 0.04$ \\
\hline 53790 & & $15.98 \pm 0.01$ & $15.15 \pm 0.01$ & $14.19 \pm 0.01$ & $13.25 \pm 0.01$ & $12.09 \pm 0.07$ & $11.01 \pm 0.03$ & $10.36 \pm 0.04$ \\
\hline 53792 & & $16.16 \pm 0.01$ & $15.27 \pm 0.01$ & $14.31 \pm 0.01$ & $13.38 \pm 0.01$ & $12.08 \pm 0.07$ & $11.07 \pm 0.03$ & $10.41 \pm 0.04$ \\
\hline 53796 & & & & & & $12.08 \pm 0.07$ & $11.08 \pm 0.03$ & $10.44 \pm 0.04$ \\
\hline 53797 & & $16.23 \pm 0.01$ & $15.38 \pm 0.01$ & $14.40 \pm 0.01$ & $13.45 \pm 0.01$ & $12.35 \pm 0.07$ & $11.14 \pm 0.03$ & $10.64 \pm 0.04$ \\
\hline 53798 & & $16.48 \pm 0.01$ & $15.60 \pm 0.01$ & $14.59 \pm 0.01$ & $13.61 \pm 0.01$ & $12.37 \pm 0.07$ & $11.30 \pm 0.03$ & $10.63 \pm 0.04$ \\
\hline 53801 & & $16.29 \pm 0.01$ & $15.47 \pm 0.01$ & $14.49 \pm 0.01$ & $13.54 \pm 0.01$ & $12.31 \pm 0.07$ & $11.23 \pm 0.03$ & $10.58 \pm 0.04$ \\
\hline
\end{tabular}


Table A.2. continued.

\begin{tabular}{|c|c|c|c|c|c|c|c|c|}
\hline MJD & $U$ & $B$ & $V$ & $R$ & I & $J$ & $H$ & $K$ \\
\hline 53804 & & $16.45 \pm 0.02$ & $15.57 \pm 0.02$ & $14.62 \pm 0.01$ & $13.66 \pm 0.01$ & $12.26 \pm 0.07$ & $11.24 \pm 0.03$ & $10.46 \pm 0.05$ \\
\hline 53808 & & $16.12 \pm 0.02$ & $15.33 \pm 0.02$ & $14.39 \pm 0.01$ & $13.47 \pm 0.01$ & $12.13 \pm 0.07$ & & \\
\hline 53811 & & $6.46 \pm 0.02$ & $5.64 \pm 0.02$ & $14.59 \pm 0.01$ & $3.57 \pm 0.01$ & $12.08 \pm 0.08$ & & $10.65 \pm 0.05$ \\
\hline 53814 & & $60 \pm 0.01$ & $5.78 \pm 0.02$ & $4.74 \pm 0.01$ & $13.75 \pm 0.01$ & $12.42 \pm 0.07$ & $35 \pm 0.03$ & $10.78 \pm 0.05$ \\
\hline 53817 & & $.49 \pm 0.01$ & $5.62 \pm 0.02$ & $14.63 \pm 0.01$ & $13.67 \pm 0.01$ & $12.27 \pm 0.08$ & $25 \pm 0.03$ & $10.66 \pm 0.05$ \\
\hline 53819 & & $17.10 \pm 0.02$ & $16.30 \pm 0.02$ & & & & $11.37 \pm 0.03$ & $10.80 \pm 0.05$ \\
\hline 53820 & & & & $15.16 \pm 0.01$ & $14.07 \pm 0.02$ & $12.49 \pm 0.07$ & & \\
\hline 53823 & & $.67 \pm 0.02$ & $5.86 \pm 0.02$ & $14.82 \pm 0.01$ & $13.82 \pm 0.01$ & $12.34 \pm 0.08$ & $1.24 \pm 0.03$ & $10.83 \pm 0.05$ \\
\hline 53826 & & $16.82 \pm 0.03$ & $16.07 \pm 0.02$ & $14.99 \pm 0.02$ & $3.94 \pm 0.02$ & $12.29 \pm 0.08$ & $11.23 \pm 0.03$ & $10.69 \pm 0.05$ \\
\hline 53829 & & $16.97 \pm 0.02$ & $16.13 \pm 0.02$ & $.04 \pm 0.01$ & $.00 \pm 0.02$ & $12.51 \pm 0.08$ & $11.36 \pm 0.03$ & $10.69 \pm 0.05$ \\
\hline 53832 & & $.88 \pm 0.03$ & $16.06 \pm 0.02$ & $5 \pm$ & $95 \pm 0.02$ & $33 \pm 0.08$ & $21 \pm$ & $9 \pm 0.05$ \\
\hline 35 & & \pm 0.02 & \pm 0 & 01 & 02 & $9 \pm$ & 03 & $8 \pm 0.05$ \\
\hline 53839 & & \pm 0.03 & \pm 0 & 2 & $7 \pm$ & 09 & 03 & \pm 0.05 \\
\hline 53843 & & \pm 0.02 & \pm 0 & \pm 0 & $7 \pm$ & $1 \pm 0.08$ & 03 & \pm 0.05 \\
\hline 34 & & & 5 & & & & & \pm 0.06 \\
\hline 53848 & & & & & & & & \pm 0.05 \\
\hline 53 & & & 9 & & & & & $5 \pm 0.05$ \\
\hline 53854 & & 5 & 7 & & $=0.04$ & \pm 0.13 & & \pm 0.07 \\
\hline 53860 & & \pm 0.29 & $17.23 \pm 0.13$ & & 0.05 & 10 & & \pm 0.06 \\
\hline 53861 & & $29 \pm 0.29$ & $17.85 \pm 0.28$ & $6.22 \pm 0.09$ & \pm 0.05 & $12.70 \pm 0.08$ & & $10.82 \pm 0.03$ \\
\hline 53956 & & & & & & $12.73 \pm 0.18$ & & $10.91 \pm 0.17$ \\
\hline 54111 & & 94 & 3 & & & $12.78 \pm 0.10$ & & $6 \pm 0.06$ \\
\hline 56 & & 1 & 1 & 01 & 01 & $2 \pm 0.08$ & 03 & \pm 0.05 \\
\hline & & 1 & 1 & 01 & 01 & 09 & & $8 \pm 0.06$ \\
\hline & & & 1 & & & & & .05 \\
\hline & & & $=0$ & 1 & & & & 05 \\
\hline & & 1 & \pm 0 & & & & & .04 \\
\hline 71 & & 1 & \pm 0 & 1 & & 07 & 03 & \pm 0.04 \\
\hline & & & & & & 07 & & \pm 0.04 \\
\hline 5 & & 1 & 1 & 1 & 01 & .08 & 03 & \pm 0.05 \\
\hline & & \pm 0.01 & $16.12 \pm 0.01$ & $1 \pm 0$ & $4.08 \pm 0$ & $11.75 \pm 0.06$ & 03 & $10.78 \pm 0.04$ \\
\hline & & $94 \pm 0.01$ & $16.11 \pm 0.01$ & $15.16 \pm 0.01$ & $.15 \pm 0.01$ & $12.47 \pm 0.07$ & $11.43 \pm$ & $10.83 \pm 0.04$ \\
\hline 54487 & & $.94 \pm 0.02$ & $16.10 \pm 0.01$ & $15.12 \pm 0.01$ & $4.08 \pm 0.01$ & $12.45 \pm 0.07$ & $11.37 \pm$ & $10.87 \pm 0.04$ \\
\hline 54490 & & $.08 \pm 0.02$ & $16.19 \pm 0.02$ & $5.18 \pm 0.02$ & $14.15 \pm$ & $12.51 \pm 0.09$ & $11.43 \pm$ & $10.80 \pm 0.05$ \\
\hline 54496 & & $.76 \pm 0.01$ & $15.91 \pm 0.01$ & $14.92 \pm 0.01$ & $13.90 \pm 0.01$ & $12.38 \pm 0.07$ & $11.39 \pm 0.03$ & $10.77 \pm 0.04$ \\
\hline 54499 & & $16.80 \pm 0.01$ & $15.98 \pm 0.01$ & $14.97 \pm 0.01$ & $13.98 \pm 0.01$ & $12.49 \pm 0.08$ & $11.41 \pm 0.03$ & $10.80 \pm 0.04$ \\
\hline 54514 & & $.71 \pm 0.04$ & $16.86 \pm 0.03$ & $15.71 \pm 0.03$ & $14.52 \pm 0.01$ & $12.66 \pm 0.09$ & $11.51 \pm 0.04$ & $11.08 \pm 0.05$ \\
\hline 54522 & & $17.82 \pm 0.03$ & $16.95 \pm 0.02$ & $15.75 \pm 0.02$ & $14.55 \pm 0.01$ & $12.62 \pm 0.08$ & $11.52 \pm 0.03$ & $11.06 \pm 0.05$ \\
\hline & & & 1 & & & & & $11.21 \pm 0.05$ \\
\hline 54553 & & $18.35 \pm 0.03$ & $17.48 \pm 0.02$ & $16.18 \pm 0.02$ & $14.79 \pm 0.01$ & $12.65 \pm 0.08$ & $11.66 \pm 0.03$ & $11.19 \pm 0.05$ \\
\hline
\end{tabular}


Table A.3. Villanova magnitudes.

\begin{tabular}{cccc}
\hline \hline MJD & $V$ & $R$ & $I$ \\
\hline 53398 & $13.66 \pm 0.05$ & $12.81 \pm 0.06$ & $12.00 \pm 0.02$ \\
53399 & $13.55 \pm 0.02$ & $12.71 \pm 0.03$ & $11.91 \pm 0.01$ \\
53401 & $13.62 \pm 0.07$ & $12.75 \pm 0.05$ & $11.95 \pm 0.04$ \\
53402 & $13.70 \pm 0.06$ & $12.81 \pm 0.05$ & $12.02 \pm 0.01$ \\
53403 & $13.81 \pm 0.06$ & $12.92 \pm 0.05$ & $12.11 \pm 0.02$ \\
53406 & $13.64 \pm 0.03$ & $12.78 \pm 0.05$ & $11.97 \pm 0.02$ \\
53418 & $13.58 \pm 0.33$ & $12.49 \pm 0.09$ & $11.71 \pm 0.04$ \\
53428 & $13.09 \pm 0.07$ & $12.28 \pm 0.04$ & $11.53 \pm 0.03$ \\
53435 & $12.77 \pm 0.02$ & $11.95 \pm 0.01$ & $11.18 \pm 0.05$ \\
53436 & $12.57 \pm 0.02$ & $11.78 \pm 0.02$ & $11.05 \pm 0.01$ \\
53444 & $13.23 \pm 0.03$ & $12.37 \pm 0.03$ & $11.57 \pm 0.03$ \\
53445 & $13.21 \pm 0.03$ & $12.36 \pm 0.03$ & $11.56 \pm 0.03$ \\
53621 & $13.58 \pm 0.04$ & $12.68 \pm 0.03$ & $11.86 \pm 0.02$ \\
53625 & $13.99 \pm 0.03$ & $13.07 \pm 0.05$ & $12.20 \pm 0.02$ \\
53634 & $13.52 \pm 0.06$ & $12.64 \pm 0.03$ & $11.80 \pm 0.04$ \\
53641 & $13.10 \pm 0.04$ & $12.26 \pm 0.02$ & $11.50 \pm 0.02$ \\
53659 & $13.49 \pm 0.04$ & $12.62 \pm 0.03$ & $11.79 \pm 0.03$ \\
53674 & $13.44 \pm 0.02$ & $12.60 \pm 0.02$ & $11.82 \pm 0.02$ \\
53707 & $14.09 \pm 0.03$ & $13.17 \pm 0.04$ & $12.34 \pm 0.03$ \\
53711 & $14.82 \pm 0.09$ & $13.89 \pm 0.08$ & $12.99 \pm 0.03$ \\
53712 & $14.74 \pm 0.13$ & $13.81 \pm 0.06$ & $12.89 \pm 0.04$ \\
53718 & $14.45 \pm 0.12$ & $13.57 \pm 0.11$ & $12.69 \pm 0.05$ \\
53748 & $15.12 \pm 0.22$ & $14.20 \pm 0.16$ & $13.28 \pm 0.11$ \\
53751 & $15.12 \pm 0.34$ & $14.36 \pm 0.19$ & $13.41 \pm 0.09$ \\
53754 & $14.49 \pm 0.16$ & $13.55 \pm 0.04$ & $12.68 \pm 0.04$ \\
53755 & $14.61 \pm 0.04$ & $13.69 \pm 0.06$ & $12.78 \pm 0.03$ \\
53762 & $14.16 \pm 0.08$ & $13.30 \pm 0.08$ & $12.44 \pm 0.06$ \\
53773 & $14.99 \pm 0.08$ & $14.11 \pm 0.07$ & $13.19 \pm 0.05$ \\
53774 & $14.78 \pm 0.11$ & $13.92 \pm 0.07$ & $13.00 \pm 0.02$ \\
53785 & $14.83 \pm 0.12$ & $13.94 \pm 0.08$ & $13.06 \pm 0.06$ \\
53790 & $15.03 \pm 0.07$ & $14.12 \pm 0.06$ & $13.18 \pm 0.03$ \\
53793 & $15.39 \pm 0.14$ & $14.48 \pm 0.10$ & $13.52 \pm 0.03$ \\
53795 & $14.87 \pm 0.08$ & $14.09 \pm 0.05$ & $13.19 \pm 0.03$ \\
53800 & $15.23 \pm 0.10$ & $14.37 \pm 0.08$ & $13.42 \pm 0.05$ \\
53802 & $15.16 \pm 0.08$ & $14.24 \pm 0.05$ & $13.36 \pm 0.03$ \\
\hline & & &
\end{tabular}

\section{Appendix B: Details on the Spitzer data reduction}

\section{B.1. IRAC}

We used the MOsaicker and Point source EXtractor (MOPEX) software release of June 2007. We started from the Basic Calibrated Data (BCD) individual images to produce mosaics with a (native) pixel size of 1 '.'22. For the data taken in sub-array mode (PIDs 3716 and 41019), we collapsed the 3-dimensional image BCDs into 2-dimensional BCDs by using, for each pixel, the median of the 64 planes. For the uncertainty BCD files, we used the standard deviation of the 64 planes of the BCD file instead of using the input uncertainties. This method allows the removal of most particle hits and the ingestion of the collapsed BCDs in MOPEX. We then used aperture photometry, centered on V1118 Ori, using an extraction radius of 10 pixels (12'.2), and a concentric annulus of radii 12 and 15 pixels (we used radii of 10 and 12.5 pixels for the IRAC4 band at $8.0 \mu \mathrm{m}$ because of the strength of the background intensity at this wavelength for radii larger than 12.5 pixels. Indeed, the background is dominated by emission caused by the nearby Herbig Ae star V372 Ori; this effect is less prominent in the other IRAC bands, but the presence of the Herbig star limited us to outer radii of 15 pixels). No aperture correction is needed for an extraction circle radius of 10 pixels (according to the IRAC data handbook v3.0).

\section{B.2. MIPS}

We used the BCD files where V1118 Ori was on the detector as input files for the MOPEX pipeline. A native pixel size of 2 '.5 was used to create the mosaic, and we used an extraction radius of $13^{\prime \prime}$ centered on V1118 Ori to derive the aperture photometry. We used an annulus of radii $15^{\prime \prime}$ and $20^{\prime \prime}$ for the background, and finally used an aperture correction factor of 1.17 (from Table 3.12 of the MIPS data handbook, v. 3.3.1). We also investigated the background with a different method, i.e., to calculate the background in a nearby region using a circle with radius 13". Indeed, the MIPS24 background near V1118 Ori is highly inhomogeneous. Notice that, while the uncertainty in the flux density is on the order $0.5 \mathrm{mJy}$, we estimate the true uncertainty, mainly because of the inhomogeneity of the background and the difficulty of finding a representative background region, to be closer to $15-20 \mathrm{mJy}$.

\section{B.3. IRS}

We extracted the IRS spectra using the Spitzer IRS Custom Extraction (SPICE) v2.1.2 software and the post-BCD co-added 2-D images (pipeline version S15.3.0 for SL/SH and S17.2.0 for LH/LL for the outburst data; S18.5.0 for all post-outburst spectra). We investigated in detail the methodology for subtracting the background in the IRS spectra. Indeed, for the low-resolution modules, we have investigated different techniques for estimating the background contamination. Firstly, we used the standard technique that takes the image from nod $\mathrm{A}$, subtracts the nod $\mathrm{B}$ image, and then the net (A-B) spectrum of V1118 Ori is extracted with SPICE. The same is then done for the net (B-A) spectrum, and both net spectra are averaged. Unfortunately, this technique is unsatisfactory because of the inhomogeneity of the background emission along the cross-dispersion axis of the slit. A second technique consisted in using the images taken for the SL2 ${ }^{9}$ nods and use the source-free region in the SL1 image for the background of the SL1 nods. This method removes any contamination by V1118 Ori in the SL1 image, but the downside is that the region of the sky covered by the SL1 slit (during the SL2 observations) is away from V1118 Ori. Thus, the measured background may not represent the background near V1118 Ori. A third technique consisted in using a background region near V1118 Ori obtained during the observed nod. This technique allows to get a better estimate for the background near V1118 Ori, but has the disadvantage that V1118 Ori may contaminate the background, especially at longer wavelengths, since the standard extraction width increases with increasing wavelength, to take into account the increasing size of the point spread function of a point source. For the February 2005 observation, technique \#2 proved better for SL1, while technique \#3 was better for SL2. We used the PAH emission at 6.2 and $11.3 \mu \mathrm{m}$ (coming mostly from the diffuse background emission) to check that the background emission was adequately removed. For the March 2005 observation, we used technique \#3 for both SL slits, as technique \#2 gave an excess in the PAH at $11.3 \mu \mathrm{m}$ in the SL1 slit. For the post-outburst observations, we used technique \#3 for both SL slits and also for the LL slits (in this case, the background subtraction is more difficult for $\lambda>30 \mathrm{mic}$ ).

For the high-resolution modules, we used the standard approach to average the spectra from both nods using the full slit aperture. For the post-outburst spectra, we used the

\footnotetext{
9 For clarity, we explain the second technique for SL2 only in the main text. For the SL1 data, simply permute SL2 and SL1 in the text.
} 

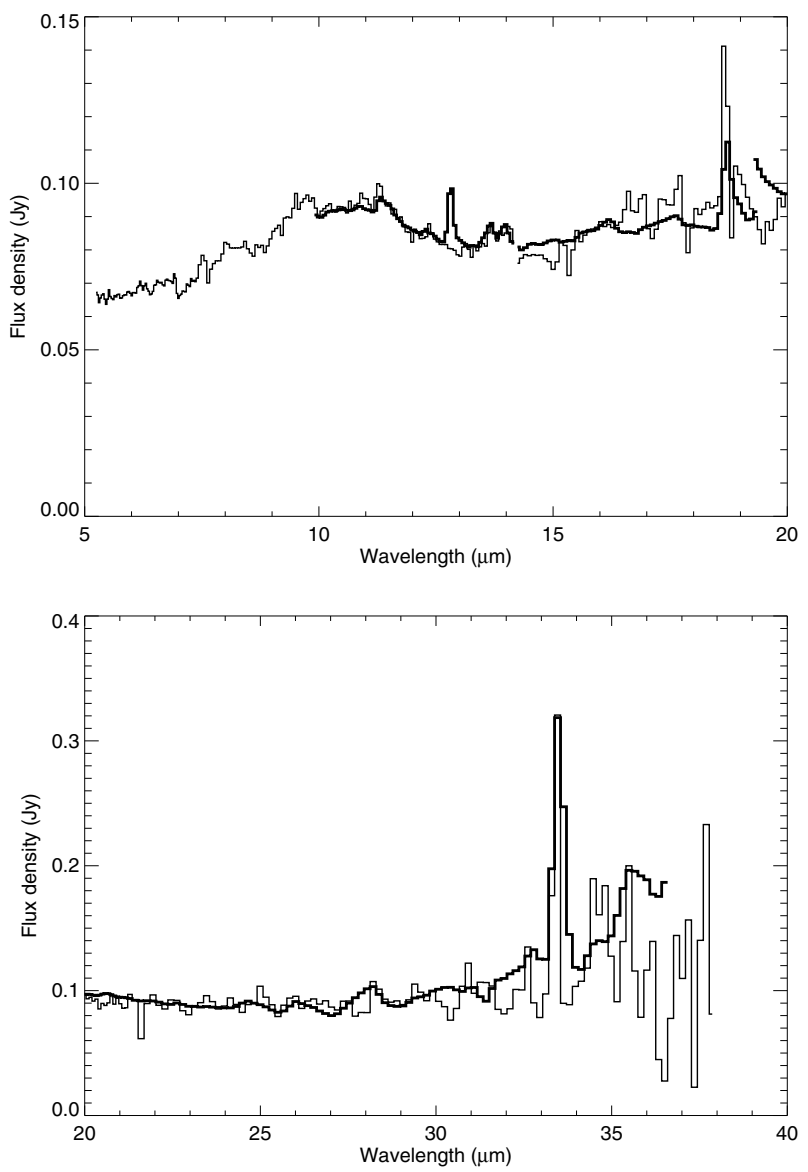

Fig. B.1. Spitzer IRS high-resolution post-outburst spectra (thick curve) degraded to the resolution of the SL and LL modules. The latter data are shown as well (thin curve). Note the good agreement in the continuum and for most lines, except for [Ne II].

accompanying background spectra to obtain the backgroundsubtracted spectra. This procedure worked nicely for the SH module (i.e., up to $19.35 \mu \mathrm{m}$ ), as demonstrated by the good subtraction of the PAH emission at $11.3 \mu \mathrm{m}$. Notice that the $\mathrm{H}_{2} \mathrm{O}-0$ $S$ (1) $\lambda 17.0348 \mu \mathrm{m}$ line was apparently completely subtracted, while the $\mathrm{H}_{2} \mathrm{O}-0 \mathrm{~S}$ (2) $\lambda 12.2786 \mu \mathrm{m}$ line is faintly detected, We also detect [Ne II] $\lambda 12.8136 \mu \mathrm{m}\left({ }^{2} P_{1 / 2}-{ }^{2} P_{3 / 2}\right)$ and [S III] $\lambda 18.7130 \mu \mathrm{m}\left({ }^{3} P_{2}-{ }^{3} P_{1}\right)$ in the background-subtracted spectrum (we will come back to this issue below). Furthermore, the silicate feature and continuum flux up to $14 \mu \mathrm{m}$ are consistent with the SL flux, suggesting that the background subtraction was not too far off. For the LH module, we subtracted only $95 \%$ of the background flux for the post-outburst data. Indeed, at the wavelengths covered by the LH, the background dominates the emission and is highly position-dependent. Although our background pointing was close to V1118 Ori, there is considerable inhomogeneity in the diffuse emission of the Orion nebula, as observed in the 8.0 and $24 \mu \mathrm{m}$ images. Figure B. 2 shows the MIPS $24 \mu \mathrm{m}$ images together with the "field-of-views" of the IRS slits during the first outburst observation and in late 2008. We used the [Si II] $\lambda 34.815 \mu \mathrm{m}\left({ }^{2} P_{3 / 2}-{ }^{2} P_{1 / 2}\right)$ as the proxy for background subtraction for the LH module, and noticed that a scaling factor of 0.95 was better than 1.0. We detect, however, excess flux in the [S III] $\lambda 33.481 \mu \mathrm{m}\left({ }^{3} P_{1}-{ }^{3} P_{0}\right)$ line and faint excess in the $\mathrm{H}_{2} 0-0 S(0)$ $\lambda 28.2188 \mu \mathrm{m}$ line (in contrast to the $\mathrm{S}(1)$ line in the $\mathrm{SH}$ spectrum). The SH and LH spectra agree relatively well at $19.4 \mu \mathrm{m}$, suggesting again that the background subtraction was adequate.
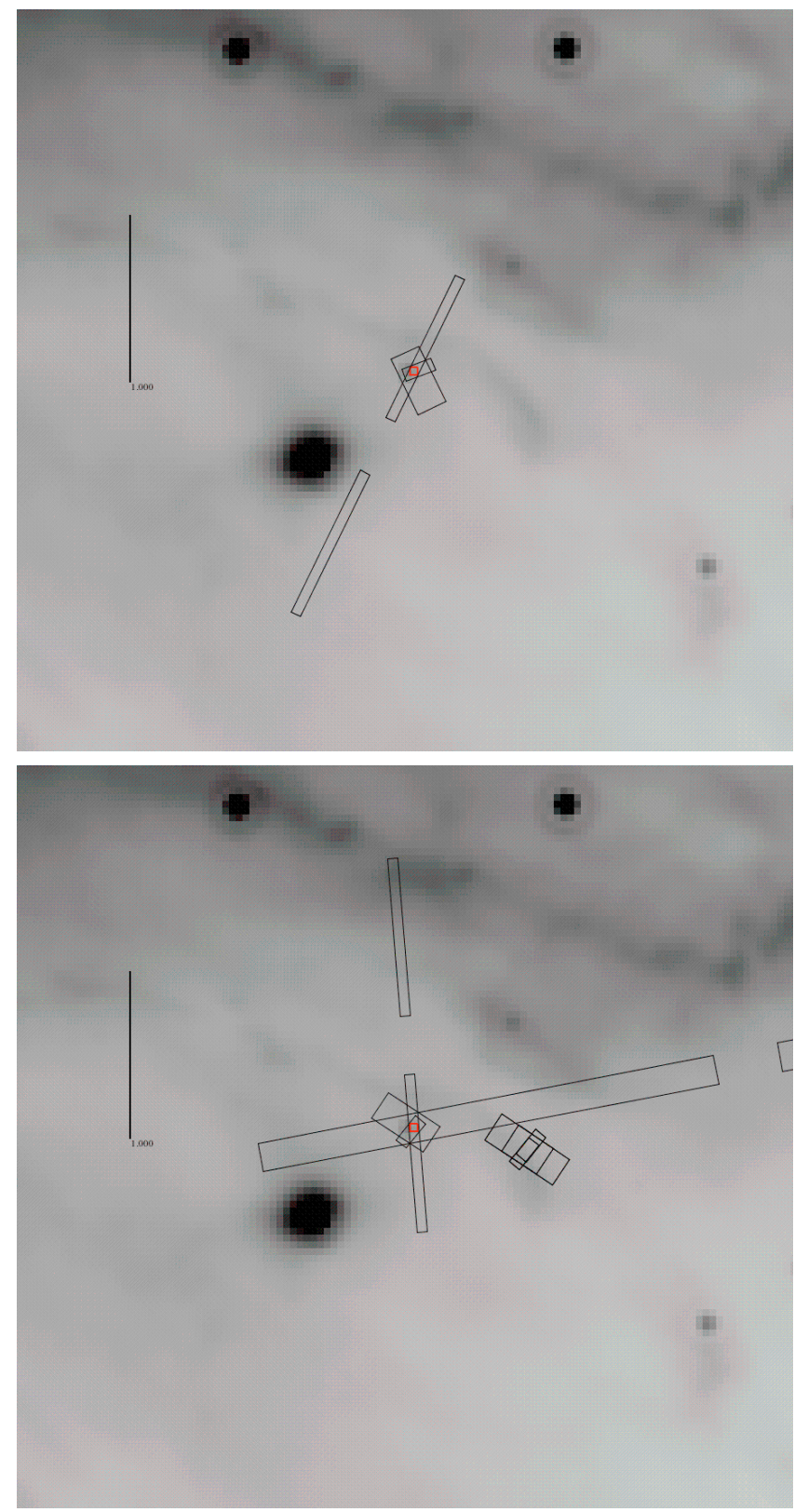

Fig. B.2. Spitzer MIPS $24 \mu \mathrm{m}$ image taken in March 2004 with overlays of the IRS modules during the observations on 2005 February 18 (top) and on 2008 November 14 after the outburst (bottom). We only show the first position of the two nods, for clarity. Both nods for the background $\mathrm{SH} / \mathrm{LH}$ observations are shown in the bottom figure. A $1^{\prime}$ scale is shown on both North-orientated images. The image scale is linear from 0 to $250 \mathrm{MJy} \mathrm{sr}^{-1}$.

We provide, however, a word of caution about the accuracy of the continuum flux level (especially above $30 \mu \mathrm{m}$ ) and in the reality of the detected flux excess in some lines, especially in the LH wavelength region. Indeed, we have scaled the background LH spectrum using the [Si II] line. However, we remind the reader that V1118 Ori is located at the center of the Orion nebula, where strong ionization and excitation of the interstellar medium takes place. Strong spatial variations of the diffuse background emission occur, which may be different for the continuum and line (mostly [Si II], [S III], [Ne II], and $\mathrm{H}_{2}$ ) emissions. Therefore, using a specific emission line to check that the background subtraction is adequate may result in an incorrect subtraction of the continuum emission of the nebula and even in 
the subtraction of another emission line! Nevertheless, the similarities of the continuum shape in the low-resolution and highresolution modules (at least below $35 \mu \mathrm{m}$ ) for the post-outburst data, the presence of line excess in [S III] in the LL spectrum and in both $\mathrm{SH}$ and LH spectra are suggestions that the background subtraction was overall accurate. The presence of [Ne II] in the SH module but not in the SL module demands, however, deeper analysis to confirm the detection of the line. We degraded the SH and LH spectra down to SL and LL resolution (Fig. B.1). The continuum shapes are well matched, but the degraded $\mathrm{SH}$ data covering the [Ne II] line shows a strong line which is not compatible with the SL data at this wavelength. This strongly suggests that the SH background [Ne II] line flux was too faint compared to the line flux near V1118 Ori and that the measured $\mathrm{SH}$ line flux is of background origin (from the Orion nebula or from diffuse emission close to V1118 Ori). In the case of $\mathrm{H}_{2}$ at $12.28 \mu \mathrm{m}$, the degraded SH spectrum is consistent with the SL spectrum: the contrast between the line and the continuum is too faint to confirm the presence of this line in the SL spectrum. The same comment applies to the other molecular hydrogen line at $28.2 \mu \mathrm{m}$. Thus, we cannot confirm that this molecule is detected in V1118 Ori's spectrum. On the other hand the [S III] lines in the degraded $\mathrm{SH}$ and LH spectra have similar peak flux densities as in the LL spectrum, indicating that these lines originate from the immediate vicinity of V1118 Ori. In summary, we believe in the detection of the [S III] lines, while we have doubts for [Ne II] and the $\mathrm{H}_{2}$ lines, but we nevertheless provide the line fluxes from the high-resolution module spectra in Table 3. In any case, in view of Spitzer spatial resolution, higher spatial resolution observations would be required to confirm the origin of the excess line emission.
We have further investigated whether the post-outburst background observations could be used for the outburst $\mathrm{SH}$ and $\mathrm{LH}$ data. In principle, the zodiacal light dominates the continuum background emission below about $40 \mu \mathrm{m}$, while the interstellar medium emission dominates above. The zodiacal contribution varies as a function of time. We have used the Spitzer Planning Observations Tool (SPOT) to determine the contribution at the three different epochs of the IRS observations and found that they were of similar level (about 20-25 $\mathrm{MJy} \mathrm{sr}^{-1}$ from 15 to $24 \mu \mathrm{m})$. For the SH spectrum, we preferred to use the postoutburst spectrum without applying a scaling factor, since the PAH feature and the $\mathrm{H}_{2}$ line $\mathrm{S}(2)$ were well subtracted (the $\mathrm{S}(1)$ line is slightly oversubtracted). For the LH spectrum, we used a scaling factor equivalent to 0.85 , i.e., about the value of the ratio of the zodiacal light contribution at $24 \mu \mathrm{m}$ at in February-March 2005 and November 2008. This ratio also cancels out relatively well the [Si II] line while the $\mathrm{H}_{2} \mathrm{~S}(0)$ line still remains detected. With this procedure, the resulting spectrum shows an increase of the flux with longer wavelengths, perhaps coming from an envelope, but this increase is not detected in the post-outburst data, casting some doubt on the accuracy of the continuum shape in the SH and LH outburst spectra above $14 \mu \mathrm{m}$. Since we have no outburst LL data to confirm this shape, we prefer to err on the safe side and consider that the high-resolution continuum shape for $\lambda>14 \mu \mathrm{m}$ during the outburst is unreliable. The strengths of the emission lines may also be affected by unreliable background reduction; indeed the flux levels are generally larger than in the post-outburst spectrum (except for [Ne II], but in this case, the line is probably not originating in the direct vicinity of V1118 Ori). Line fluxes from the high-resolution module data in outburst are also given in Table 3. 